\title{
Magnetização Espontânea em Modelos de Ising Uni-Dimensionais com Interação de Longo Alcance
}

\author{
por \\ Leonardo Cavalcanti de Mélo
}

Orientador: Leandro Martins Cioletti

Brasília

2014 


\section{Leonardo Cavalcanti de Mélo}

\section{Magnetização Espontânea em Modelos de Ising Uni-Dimensionais com Interação de Longo Alcance}

Dissertação apresentada ao Programa de Pós-Graduação em Matemática da Universidade de Brasília como requisito parcial à obtenção do Título de Mestre em Matemática.

Orientador: Leandro Martins Cioletti 


Melo, Leonardo C.
Magnetização Espontânea em Modelos
de Ising Uni-Dimensionais com Interação de
Longo Alcance
$\quad$ 89 páginas
Dissertação (Mestrado em Matemática).
Universidade de Brasília. Programa de Pós-
Graduação em Matemática.
1. Modelo de Ising
2. Mecânica Estatística
I. Universidade de Brasília. Departamento de
Matemática.

\section{Comissão Julgadora:}

Leandro Martins Cioletti

UnB

Chang Chung Yu Dorea

UnB

Artur Oscar Lopes

UFRGS 


\section{Agradecimentos}

Gostaria de agradecer a todos os colegas, alunos da matemática da UnB, que alimentam um ambiente de discussão aberto e cooperativo na instituição. O incentivo dos colegas foi muito importante para manter meu ânimo durante o curso.

Agradeço a meus professores de álgebra, Elaine e Leandro. Minha aprovação naquele curso se deve à ajuda deles. Agradeço também aos "irmãos" de orientação Roberto, Jamer e Josimar por todas as discussões matemáticas, mas também pelo miolo de pote.

Agradeço aos demais colegas de turma, com quem tive muitas oportunidades de conversar e aprender sobre matemática e outras coisas, especialmente às incentivadoras Lury e Chris. Também ao meu camarada Valter, por vir à capital do cerrado e dar-me a oportunidade de conhecê-lo. Ainda terei a chance de aprender um pouco de geometria com ele.

Agradeço ao professores que me guiaram neste curso e nos cursos prévios: Claus Akira, Gilberto Fernandes Vieira, Daniele Baratela, Pedro Roitman, Noraí Rocco, Ricardo Ruviaro, Cátia Gonçalves e Leandro Cioletti.

Agradeço aos envolvidos na administração da pós-graduação em matemática da UnB, especialmente à Bruna, que sempre esteve presente para resolver todas as burocracias da forma mais simples possível. Também à Profa. Liliane, coordenadora da pós-graduação durante boa parte do meu curso, pela sua dedicação. Durante sua administração ela sempre procurou as melhores alternativas para permitir a mim e aos demais colegas o máximo de tranquilidade com questões práticas para que pudéssemos nos dedicar quase integralmente aos estudos. Devemos bastante a ela.

Agradeço a meus superiores no Ministério do Planejamento por admitirem a licença que me permitiu investir em minha formação. Foram responsáveis diretos por isso Márcio Oliveira e Luiz Guilherme Henriques. Também aos colegas de trabalho por cobrarem a minha conclusão. Leila Frossard foi a fiscal mais ativa e não me deixou atrasar mais um minuto.

Agradeço aos meus familiares, especialmente a Cássia, pela compreensão e apoio neste projeto. Envolvi-me neste curso a despeito da desaprovação de boa parte dos conhecidos. O valor que cultivamos pelos estudos herdado de Bartolomeu e Beatriz me impulsiona a sempre procurar novidades. Simplesmente não tenho outra opção. Como está pichado no túnel da 215 norte: "Opte por aquilo que faz seu coração vibrar" - Osho.

Agradeço ao meu orientador, Prof. Leandro Cioletti, pela sua paciên- 
cia e solicitude. Desde que o conheci, vejo o Prof. Leandro trabalhar incansavelmente, sempre animado com o próximo desafio. Obrigado por admitir orientar-me, mesmo ciente das minhas restrições práticas. Obrigado também pelas inúmeras contribuições para melhorar este texto. Não fosse seu suporte e incentivo, provavelmente eu não conseguiria concluir este trabalho. 


\section{Resumo}

Nesta dissertação de mestrado expomos alguns resultados clássicos relativos ao modelo de Ising em uma dimensão. O tópico final é a existência de transição de fase nos sistemas com interações de longo alcance (alcance infinito) cujo Hamiltoniano é formalmente dado por

$$
H=-\sum_{i, j: i<j} \frac{1}{|i-j|^{\alpha}} \sigma_{i} \sigma_{j}
$$

com $1<\alpha<2$. A exposição desse tema é baseada na referência [5].

Com objetivo de manter o texto auto-contido, foram incluídos vários capítulos introdutórios. Nesses capítulos iniciais são construídas algumas ferramentas que permitirão a abordagem do tema principal no último capítulo. Todas essas construções são baseadas na literatura disponível e não há inovações significativas. Dentre os tópicos preliminares apresentados estão a definição rigorosa do Modelo de Ising a volumes finitos, bem como do modelo de Curie-Weiss e do modelo Hierárquico. Para o modelo de Curie-Weiss a magnetização e a temperatura crítica são calculados explicitamente.

Também são apresentadas as demonstrações para: algumas desigualdades de correlação; a existência do limite termodinâmico da magnetização de modelos ferromagnéticos; e uma relação entre a magnetização quadrática e a magnetização espontânea.

Palavras-chave: Modelo de Ising unidimensional, Modelo de Curie-Weiss, Modelo Hierárquico, Sistemas ferromagnéticos, Desigualdades de correlação, Magnetização quadrática, Transição de fase. 


\section{Abstract}

In this master thesis we present some classical results relative to the one-dimensional Ising model. The main subject is the existence of phase transition in systems with long range interactions (infinite range) and Hamiltonian formally given by

$$
H=-\sum_{i, j: i<j} \frac{1}{|i-j|^{\alpha}} \sigma_{i} \sigma_{j},
$$

where $1<\alpha<2$. The exposition of the main theme is completely based on the reference [5].

Aiming to keep this text self-contained, we included some introductory chapters. In these background chapters we build some tools needed to handle the main theme on the last chapter. All of these constructions are based on the available literature and no important innovation is added. Among these preliminary topics are rigorous definitions of the Ising, CurieWeiss and Hierarchical models. The exact value of the magnetization and the critical temperature are explicitly computed for the Curie-Weiss model.

We also provided the proofs for: some correlation inequalities; existence of the thermodynamic limit for magnetization in ferromagnetic models; and the relation between mean-squared magnetization and spontaneous magnetization.

Keywords: One-dimensional Ising Model, Curie-Weiss model, Hierarchical model, Ferromagnetic systems, Correlation Inequalities, Mean-squared magnetization, Phase Transition. 


\section{Apresentação}

O texto está dividido em 4 capítulos. O Capítulo 1, de introdução, traz um breve histórico referente ao modelo de Ising, define o modelo em uma caixa finita $\Lambda \in \mathbb{Z}$ e cita alguns dos principais resultados referentes a esse modelo.

O Capítulo 2 trata de alguns resultados básicos importantes, exibindo demonstrações das três desigualdades de correlação de uso recorrente neste texto, as Desigualdades de FKG, GKS-2 e GHS. As duas primeiras citadas desigualdades, são utilizadas nas últimas seções desse capítulo para demonstrar a existência do limite termodinâmico da magnetização. Também é demonstrada uma relação entre a magnetização quadrática e a magnetização específica, que será importante para o desenvolvimento seguinte.

O Capítulo 3 trata dos modelos de Ising com interação translacionalmente invariante. Há um tratamento extensivo do modelo de Curie-Weiss para o qual são calculadas analiticamente as seguintes funções termodinâmicas: pressão, energia livre e magnetização. Também é obtido rigorosamente a temperatura crítica e demonstrado que a magnetização nesse modelo nunca é inferior à magnetização de um outro modelo de Ising arbitrário com $M_{0}=\sum_{i} J_{i j}$ constante, desde que a constante $M_{0}$ seja a mesma em ambos os modelos. Esse último resultado garante a existência de uma região de temperaturas onde magnetização no modelo de Ising é nula para todos os sistemas somáveis $\left(M_{0}<\infty\right)$, em outras palavras, garante que a temperatura crítica é finita.

Por último, são apresentados outros modelos com interação do tipo lei de potência, $J_{i j}=J(|i-j|)=|i-j|^{\alpha}$. São citados os resultados já conhecidos em 1969 sobre os modelos com esse tipo de interação, bem como consequências desses resultados a um espectro maior de modelos.

O Capítulo 4 traz a demonstração primeiramente apresentada em [5] da positividade da magnetização quadrática abaixo de uma temperatura crítica positiva em modelos com interação de longo alcance da forma $J_{i, j}=|i-j|^{\alpha}$, desde que $1<\alpha<2$. Aspectos do Modelo Hierárquico utilizados na demonstração serão vistos em detalhe. Nesse capítulo são usados os resultados do Capítulo 2 para demonstrar que a positividade da magnetização quadrática a campo externo nulo implica na positividade da magnetização espontânea. Juntando com as conclusões do Capítulo 3 a respeito do Modelo de Curie-Weiss, vamos concluir que qualquer modelo com $1<\alpha<2$ tem temperatura crítica positiva e finita. 


\section{Sumário}

1 Contextualização 1

1.1 Introdução $\ldots \ldots \ldots \ldots \ldots \ldots \ldots$

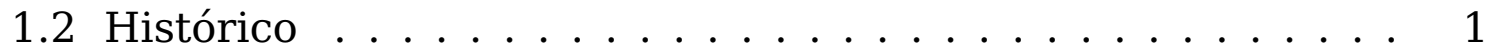

1.3 Definição do Modelo a Volume Finito . . . . . . . . . . . . . 3

1.4 Resultados Clássicos . . . . . . . . . . . . . . . . . . 7

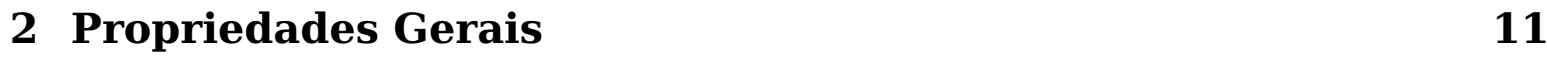

2.1 Introdução . . . . . . . . . . . . . . . . . . . 11

2.2 Desigualdades de Correlação . . . . . . . . . . . . . . . . 12

2.3 Condições Exteriores . . . . . . . . . . . . . . . . . . 28

2.4 Magnetização a Volume Finito . . . . . . . . . . . . . . . . 29

2.5 Algumas Propriedades de $S_{N} / N \ldots \ldots \ldots$. . . . . . 34

3 Modelos com Interações Translacionalmente Invariante 39

3.1 Introdução . . . . . . . . . . . . . . . . . . . . . 39

3.2 Modelo de Curie-Weiss . . . . . . . . . . . . . . . . . . 40

3.3 A Pressão do Modelo de Curie-Weiss . . . . . . . . . . . . . . 41

3.4 Curie-Weiss e a Derivada da Pressão . . . . . . . . . . . . . . 47

3.5 Magnetização Espontânea no Modelo de Curie-Weiss . . . . . 53

3.6 Comparação de um Modelo de Ising com Curie-Weiss . . . . . 58

3.7 Acoplamento $J_{i j}=|i-j|^{-\alpha}$ : Casos $\alpha \leq 1 \mathrm{e} \alpha>2 \ldots \ldots$. . . 65

3.8 Comparando Modelos com Interações do Tipo $J(|i-j|)$. . . . 67

4 Transição de Fase com Interações de Longo Alcance 69

4.1 Introdução . . . . . . . . . . . . . . . . . . . . 69

4.2 O Modelo Hierárquico . . . . . . . . . . . . . . . . 70

4.3 Comparando os Modelos Hierárquico e de Ising . . . . . . . 76

4.4 Magnetização Quadrática e Magnetização Espontânea . . . . 77

4.5 Magnetização Quadrática Não-nula em Baixas Temperaturas 80

\begin{tabular}{lr}
\hline Referências Bibliográficas & 87
\end{tabular} 


\section{Capítulo 1}

\section{Contextualização}

\subsection{Introdução}

Este capítulo trata dos aspectos históricos e das definições a serem usadas por toda a dissertação. A seção 1.2 traz uma breve apresentação do meio histórico e dos problemas físicos que deram origem ao modelo de Ising. A seção seguinte, 1.3, traz a definição formal do modelo em uma caixa finita e define outras estruturas importantes para a apresentação posterior, como a magnetização específica. A última seção do capítulo, 1.4, elenca alguns resultados importantes relativos ao modelo de Ising, tanto em uma dimensão (tema deste texto), como em dimensões maiores.

\subsection{Histórico}

A partir do século XX a compreensão dos materiais magnéticos tem sido de grande importância para o desenvolvimento tecnológico da humanidade. Ímãs são parte essencial de discos rígidos, caixas de som, motores elétricos, etc. Boa parte do desenvolvimento técnico referente ao magnetismo e suas aplicações deu-se a partir do fim do século XIX. No início do século passado, na década de 1920, um grande problema para a comunidade física era compreender, a partir de primeiros princípios, a existência de materiais que, após submetidos a um campo magnético externo, apresentavam uma magnetização permanente mesmo após a extinção do campo externo.

Em seus estudos sobre a propriedades magnéticas dos materiais, Pierre Curie observou em 1895 que uma classe de materiais perdia a magnetização quando aquecidos acima de uma certa temperatura crítica. Atu- 
almente essa temperatura limite é conhecida como temperatura de Curie ou ponto de Curie. Um metal com essa propriedade utilizado desde a antiguidade é a magnetita (óxido de ferro). Como o ferro e a magnetita são os materiais mais abundantes com a dita propriedade magnética, tais materiais foram denominados materiais ferromagnéticos.

$\mathrm{Na}$ tentativa de explicar os fenômenos físicos característicos dos materiais ferromagnéticos, em especial a existência de duas regiões de temperatura com propriedades magnéticas distintas, foram criados modelos microscópicos com uma interação entre seus constituintes capaz de reproduzir aqueles fenômenos macroscópicos conhecidos. Nesse sentido, os constituintes elementares mais naturais para compor os materiais ferromagnéticos eram os dipolos magnéticos. Assim, os modelos procuravam simular a interação de um grande número de dipolos magnéticos presos a uma estrutura cristalina similar à dos metais. Por esse motivo, os modelos microscópicos para descrever as propriedades magnéticas dos metais normalmente se dão sobre uma rede (grafo) periódica, que simula a estrutura cristalina, em cujos sítios são postos os dipolos magnéticos.

O modelo de Ising surgiu nesse ambiente histórico com o intuito de descrever as citadas propriedades dos materiais ferromagnéticos a partir de uma estrutura matemática simples. Ao invés de admitir dipolos magnéticos orientados em qualquer direção do espaço, cada sítio da rede é preenchido apenas com um valor inteiro, como por exemplo apenas admitindo os valores -1 e +1 . Isso é equivalente a estipular uma direção preferencial. Os dipolos ficam restritos a estar paralelos ou antiparalelos (apontando na mesma direção, mas com sentidos opostos) entre si. Essas simplificações buscavam isolar as estruturas fundamentais realmente responsáveis pelos efeitos ferromagnéticos, sendo a mudança do comportamento macroscópico a partir de uma temperatura crítica a característica mais peculiar que esses modelos teriam de prever.

Apesar de classicamente os dipolos magnéticos poderem estar orientados em qualquer direção, no âmbito da teoria quântica o modelo de Ising se mostrou ainda mais adequado a descrever os sistemas para os quais foi inicialmente elaborado, já que a interação entre os dipolos magnéticos (daqui em diante, spins) é realmente restrita a um conjunto finito de valores. Para o caso da interação entre dois elétrons, a energia de interação é de fato proporcional a +1 ou -1 , dependendo de os spins estarem alinhados paralela ou antiparalelamente.

Embora feito sob medida para explicar as propriedades magnéticas de 
certos materiais, o modelo de Ising se mostrou suficientemente versátil para modelar diversos outros problemas em que interações entre constituintes fundamentais resulta em uma mudança do comportamento macroscópico do sistema. Já recorreu-se a essa construção para modelar sistemas tão diversos como polímeros [3] e processos eleitorais [11].

\subsection{Definição do Modelo a Volume Finito}

Na tentativa de descrever os fenômenos magnéticos citados na seção anterior foi criado um modelo que atribui uma probabilidade a cada estado possível do sistema. Contudo, teria de haver alguma forma de ponderar nesse modelo a influência dos dois fatores concorrentes, a aleatoriedade (ou desordem) e a minimização da energia do sistema como um todo. Para dar conta do primeiro fator, a desordem, o modelo de Ising finito com $N$ spins permite a realização de estados que são sequências de $N$ variáveis assumindo os valores -1 ou +1 .

Para considerar o efeito da minimização da energia, foi necessário estipular uma distribuição de probabilidade compatível com esse efeito. Isto é, estipulou-se uma distribuição de probabilidade privilegiando os estados com menor energia. Para finalizar a descrição do modelo, definiu-se uma função de energia compatível com o comportamento microscópico esperado dos spins, ou melhor, uma função que atribui menor energia a spins alinhados na mesma direção.

O modelo de Ising unidimensional no volume $\Lambda=\mathbb{Z} \cap[-N, N]$ pode ser definido como sendo o processo estocástico $\left\{X_{t}\right\}_{t \in T}$ definido no espaço de probabilidade $\left(\Omega_{\Lambda}, \mathcal{F}_{\Lambda}, \mathbb{P}_{\Lambda}\right)$, onde o conjunto de índices $T=\Lambda$, o espaço amostral $\Omega_{\Lambda}=\{-1,1\}^{\Lambda}, \mathcal{F}_{\Lambda}$ é a $\sigma$-álgebra das partes de $\Omega_{\Lambda}$ e a medida de probabilidade $\mathbb{P}_{\Lambda}$ é dada adiante em (1.1).

Para cada $t \in \Lambda$ a variável aleatória $X_{t}: \Omega_{\Lambda} \rightarrow\{-1,1\}$ é definida no ponto $\left(\sigma_{-N}, \ldots, \sigma_{N}\right) \equiv \sigma \in \Omega_{\Lambda}$ por $X_{t}(\sigma)=\sigma_{t}$. No contexto de Mecânica Estatística as variáveis aleatórias $X_{t}$ 's são chamadas de spins. Neste texto vamos adotar a notação consagrada da área e denotar a variável aleatória $X_{t}$ por $\sigma_{t}$. Uma observação sobre essa notação é que frequentemente os elementos de $\Omega_{\Lambda}$ são chamados de $\sigma \equiv\left(\sigma_{-N}, \ldots, \sigma_{N}\right)$ e assim a variável aleatória $\sigma_{t}$ calculada neste ponto é dada por $\sigma_{t}(\sigma)=\sigma_{t}$. Claramente o lado direito desta igualdade não é a variável aleatória $\sigma_{t}$, e sim seu valor no ponto $\sigma$. É claro que para $\omega \in \Omega_{\Lambda} \operatorname{com} \omega=\left(\omega_{-N}, \ldots, \omega_{N}\right)$ temos $\sigma_{t}(\omega)=\omega_{t}$ e neste caso não há nenhuma chance de confusão. Observamos porém que ficará claro 
pelo contexto se $\sigma_{t}$ denota uma variável aleatória ou o valor da $t$-ésima coordenada do vetor $\sigma=\left(\sigma_{-N}, \ldots, \sigma_{N}\right)$.

O conjunto $\Omega_{\Lambda}$ também é chamado de espaço de estados e um elemento $\sigma \equiv\left(\sigma_{-N}, \ldots, \sigma_{N}\right)$ desse conjunto é interpretado como um estado do sistema de partículas na rede $\Lambda=[-N, N] \cap \mathbb{Z}$. A probabilidade do sistema ser encontrado no estado $\sigma$ é dado por:

$$
\mathbb{P}_{\Lambda}(\sigma)=\frac{\exp \left[-\beta H_{\Lambda}(\sigma)\right]}{Z_{\Lambda}}, \quad \text { onde } Z_{\Lambda}=\sum_{\omega \in \Omega_{\Lambda}} \exp \left[-\beta H_{\Lambda}(\omega)\right] .
$$

A função $H_{\Lambda}$ é conhecida como hamiltoniano do sistema. $H_{\Lambda}(\sigma)$ é a energia do sistema no estado $\sigma$ e no modelo de Ising defini-se como:

$$
H_{\Lambda}(\sigma) \equiv H_{\Lambda}\left(\sigma_{-N}, \ldots, \sigma_{N}\right)=-\sum_{-N \leq i<j \leq N} J_{i j} \sigma_{i} \sigma_{j}-\sum_{i=-N}^{N} h_{i} \sigma_{i}
$$

onde $\left\{J_{i j}\right\}$ e $\left\{h_{i}\right\}$ são constantes reais fixadas.

O modelo de Ising $D$-dimensional a volume finito é definido de maneira análoga, a diferença é que o conjunto de índices $T$ do processo $\left\{X_{t}\right\}_{t \in T}$ é agora da forma $T=\Lambda=\mathbb{Z}^{D} \cap[-N, N]^{D}$.

A medida de probabilidade $\mathbb{P}_{\Lambda}$ definida em (1.1) segue a tradição da termodinâmica. Para pesar as influências concorrentes da aleatoriedade e da minimização de energia a probabilidade de um certo estado $\sigma$ ser observado diminui exponencialmente com sua energia $H_{\Lambda}(\sigma)$, o que dá origem a expressão

Como podemos perceber, o parâmetro $\beta$ entra em (1.1) como fator multiplicativo da energia, definindo precisamente como uma unidade de energia extra diminui a probabilidade de um determinado estado. Em outras palavras, $\beta$ define a escala da energia. $\beta$ é uma constante dependente apenas de um parâmetro físico, a temperatura do sistema. Visto que $\beta=\frac{1}{k_{B} T}$, comumente $\beta$ é denominado simplesmente inverso da temperatura, apesar de, a rigor, ser proporcional ao inverso da temperatura. A constante de proporcionalidade $k_{B}$ é conhecida por constante de Boltzmann. Essa é a constante fundamental que traduz a relação entre energia e temperatura. Ela define quanto a variação de uma unidade de temperatura influi na minimização de energia, convertendo a unidade de medida de temperatura em unidades de energia. Em (1.1), a dependência de $\mathbb{P}_{\Lambda}$ com respeito as constantes $\beta>0,\left\{h_{i}\right\},\left\{J_{i j}\right\}$ foram omitidas para não carregar a notação. Quando for necessário explicitar tal dependência, escreveremos, por exemplo, $\mathbb{P}_{\Lambda, \beta, h}$ quando $h_{i}=h \in \mathbb{R}$ para todo $i \in \Lambda$. 
Observe que $Z_{\Lambda}$ funciona como uma constante de normalização e é definida para garantir $\sum_{\sigma \in \Omega_{\Lambda}} \mathbb{P}(\sigma)=1$. Assim, a presença de $Z_{\Lambda}$ no denominador em (1.1) garante que $\mathbb{P}_{\Lambda}$ é uma medida de probabilidade. $Z_{\Lambda}$ define como a probabilidade é particionada entre os estados $\sigma \in \Omega_{\Lambda}$. Pensando nas constantes $\beta,\left\{h_{i}\right\}$, etc como parâmetros, podemos olhar para $Z_{\Lambda}$ como uma função desses parâmetros. Com essa interpretação, $Z_{\Lambda}$ é costumeiramente referida como função de partição e, como mencionado acima, é dada pela expressão

$$
Z_{\Lambda}=\sum_{\omega \in \Omega_{\Lambda}} \exp \left[-\beta H_{\Lambda}(\omega)\right]
$$

Veremos mais a diante que várias informações importantes do modelo de Ising podem ser extraídas de $Z_{\Lambda}$ estudando, por exemplo, suas derivadas com respeito a $\beta$ e $h$.

Para garantir ainda que a interação privilegie os estados $\sigma \in \Omega_{\Lambda}$ com dipolos alinhados, a energia total de um estado é construída através da soma entre interações de pares de spins, sendo a interação entre um par de spins dada por

$$
H_{i, j}=-J_{i j} \sigma_{i} \sigma_{j}
$$

onde $J_{i j}$ é uma constante não negativa. O sinal de menos que a precede acarreta menor energia se ambos os spins $\sigma_{i}$ e $\sigma_{j}$ tiverem o mesmo sinal. $\mathrm{Na}$ energia do estado $\sigma$ também consta um termo referente à interação do spin no sítio $i \in[-N, N] \cap \mathbb{Z}$ com o campo magnético externo $h_{i}$ nesse mesmo sítio. Somando tudo retomamos a energia do estado $\sigma$ no modelo de Ising finito:

$$
H_{\Lambda}(\sigma)=\sum_{i \in \Lambda}\left\{\sum_{j \in \Lambda}-\frac{1}{2} J_{i j} \sigma_{i} \sigma_{j}-h_{i} \sigma_{i}\right\}, \quad \text { com } J_{k k}=0 \forall k \in \Lambda .
$$

A multiplicação por $\frac{1}{2}$ em (1.5) dá conta dessa duplicidade, assim, o somatório também pode ser equivalentemente escrito restringindo-se $i<j \mathrm{e}$ omitindo o fator $\frac{1}{2}$, forma que utilizamos anteriormente. Os sistemas com energia descrita como na equação acima, com um menos precedendo uma constante de interação não-negativa, são chamados sistemas ferromagnéticos, pois privilegiam os estados com spins alinhados paralelamente. Em diversas situações vamos tomar $h_{i} \equiv h, \forall i \in \Lambda$ e nesses casos, quando conveniente, explicitaremos a dependência de $H_{\Lambda}$ com respeito a $\left\{h_{i}\right\}$ escrevendo $H_{\Lambda, h}$. A constante $h$ é chamada de campo externo ou campo magnético externo. As constantes $J_{i j}$ são denominadas constantes de acoplamento e vamos assumir que são simétricas no seguinte sentido: $J_{i j}=J_{j i}$. 
A seção anterior nos mostrou que, ao menos em sua origem histórica, o tópico de maior interesse na análise dos sistemas aqui descritos era o comportamento da magnetização com a variação dos parâmetros externos $h$ e $\beta$. Para chegarmos ao que será chamado magnetização, vamos precisar falar do valor esperado de uma variável aleatória com respeito à medida de probabilidade $\mathbb{P}_{\Lambda}$. Dada uma função $f: \Omega_{\Lambda} \longrightarrow \mathbb{R}$ que atribui a cada estado $\sigma$ um valor real $f(\sigma)$, denotaremos por $\langle f\rangle_{\Lambda, \beta, h}$ o valor esperado de $f$ com relação à medida $\mathbb{P}_{\Lambda, \beta, h}$, isto é:

$$
\langle f\rangle_{\Lambda, \beta, h}=\sum_{\sigma \in \Omega_{\Lambda}} f(\sigma) \mathbb{P}_{\Lambda, \beta, h}=\sum_{\sigma \in \Omega_{\Lambda}} f(\sigma) \frac{\exp \left[-\beta H_{\Lambda}(\sigma)\right]}{Z_{\Lambda}}
$$

A magnetização é o valor esperado da soma de todos os spins do sistema. Assim o magnetização no modelo de Ising ao inverso da temperatura $\beta>0$ sujeito a um campo externo $h$, notação $M_{\Lambda, \beta, h}$, é dada por

$$
M_{\Lambda, \beta, h}=\left\langle S_{\Lambda}\right\rangle_{\Lambda, \beta, h}=\left\langle\sum_{i \in \Lambda} \sigma_{i}\right\rangle_{\Lambda, \beta, h}
$$

E a magnetização específica, ou magnetização por spin, é definida como sendo $M_{\Lambda, \beta, h}$ dividido pelo número de spins do sistema, ou seja:

$$
m_{\Lambda, \beta, h}=\frac{M_{\Lambda, \beta, h}}{|\Lambda|}=\frac{M_{\Lambda, \beta, h}}{2 N+1} .
$$

Tanto $M_{\Lambda, \beta, h}$ como $m_{\Lambda, \beta, h}$ são definidas em termos de funções contínuas em $\beta$ e em $h$, logo $M_{\Lambda, \beta, h}$ e $m_{\Lambda, \beta, h}$ também são contínua em $\beta$ e $h$.

Seja $(-\sigma)$ o estado obtido a partir de $\sigma$ multiplicando todas suas coordenadas por $(-1)$, isto é, $-\sigma \equiv\left(-\sigma_{-N}, \ldots,-\sigma_{N}\right)$. Segue da Equação (1.5), com $h_{i}$ constante igual a $h$, que $H_{\Lambda, \beta,-h}(\sigma)=H_{\Lambda, \beta, h}(-\sigma)$. Notando que a aplicação $\sigma \mapsto(-\sigma)$ define uma bijeção de $\Omega_{\Lambda}$ em si mesmo, podemos verificar que $\mathbb{P}_{\Lambda, \beta,-h}(\sigma)=\mathbb{P}_{\Lambda, \beta, h}(-\sigma)$. Dessa observação, de (1.6) e de (1.7) chegamos à seguinte relação:

$$
\begin{aligned}
M_{\Lambda, \beta,-h} & =\sum_{\sigma \in \Omega_{\Lambda}} \sum_{i \in \Lambda} \sigma_{i} \mathbb{P}_{\Lambda, \beta,-h}(\sigma)=\sum_{\sigma \in \Omega_{\Lambda}} \sum_{i \in \Lambda} \sigma_{i} \mathbb{P}_{\Lambda, \beta, h}(-\sigma) \\
& =-\sum_{\sigma \in \Omega_{\Lambda}} \sum_{i \in \Lambda}\left(-\sigma_{i}\right) \mathbb{P}_{\Lambda, \beta, h}(-\sigma)=-\sum_{-\sigma \in \Omega_{\Lambda}} \sum_{i \in \Lambda} \sigma_{i} \mathbb{P}_{\Lambda, \beta, h}(\sigma) \\
& =-M_{\Lambda, \beta, h} .
\end{aligned}
$$

Como era de se esperar, a inversão do campo externo simplesmente 
inverte a magnetização: $M_{\Lambda, \beta,-h}=-M_{\Lambda, \beta, h}$. Em outras palavras, a magnetização é uma função ímpar do campo externo $h$. Vimos que, além de ímpar, é contínua, logo $M_{\Lambda, \beta, 0}=0$. Assim, quando cessa o campo externo a magnetização também vai a zero suavemente. Dessa forma, o modelo de Ising a volume finito não explica a magnetização espontânea de amostras ferromagnéticas, já que numa amostra física a baixas temperaturas é observada magnetização não-nula mesmo quando o campo externo é removido do sistema, isto é, os spins se alinham, mesmo após a extinção do campo externo.

Nos capítulos seguintes, veremos que, em alguns casos, é possível quebrar a continuidade da magnetização em $h=0$ fazendo $N \longrightarrow \infty$. Isso restaura, ao menos para o caso de sistemas infinitos $(\Lambda \longrightarrow \mathbb{Z})$, a possibilidade de termos

$$
\lim _{h \rightarrow 0^{+}} \lim _{\Lambda \uparrow \mathbb{Z}} m_{\Lambda, \beta, h}>0 .
$$

Isso motiva introdução do modelo de Ising a volume infinito, na tentativa de explicar com esse modelo matemático o fenômeno da magnetização espontânea de um ferromagneto.

\subsection{Resultados Clássicos}

O modelo de Ising é, sem dúvida, um dos modelos mais importantes em Mecânica Estatística. Muitas variações do sistema definido neste capítulo já foram estudadas em detalhe. Na seção anterior, definimos esse modelo em subconjuntos finitos $\Lambda \subset \mathbb{Z}$. No entanto, é possível dar uma definição do modelo de Ising diretamente em $\mathbb{Z}$. O modelo assim construído se comporta como limite (em certo sentido), quando $\Lambda \rightarrow \mathbb{Z}$, dos modelos a volume finito que definimos na seção anterior. Neste trabalho, contudo, não apresentaremos tal construção. O leitor interessado pode encontrar uma discussão detalhada sobre esta definição em [2, 6, 12].

Como apresentamos no final da seção anterior, o tema de maior interesse neste texto é a existência de magnetização positiva quando $h \rightarrow 0^{+}$. Perceba que nesta seção adotaremos um campo externo constante, isto é, $h_{i}=h$ para todo $i \in \Lambda$. Também vimos que, devido à magnetização ser uma função ímpar do campo externo $h$, esses sistemas com magnetização espontânea necessariamente apresentam uma descontinuidade da magnetização na origem $h=0$.

Os modelos de Ising definidos em $\mathbb{Z}$ que citamos anteriormente têm 
propriedades que podem ser extraídas da sequência de modelos finitos em $\left\{\Lambda_{N}\right\}_{N}$, com $\Lambda_{N} \rightarrow \mathbb{Z}$, que o aproxima. Diz-se que o modelo a volume infinito apresenta transição de fase se

$$
\lim _{h \rightarrow 0^{+}} \lim _{\Lambda \uparrow \mathbb{Z}} m_{\Lambda, \beta, h}>0 .
$$

Esse limite é chamado magnetização a volume infinito ou simplesmente magnetização. Para o modelo de Ising Ferromagnético (longo ou curto alcance) isso é equivalente à existência de mais de uma medida de Gibbs no sentido DLR ou à existência de mais de um Limite Termodinâmico. Para maiores detalhes sobre essas equivalências veja [2, 6].

Do mesmo modo que esses sistemas a volume infinito podem ser definidos por sequências de caixas em $\mathbb{Z}$, podem também ser definidos sistemas em outros espaços nos quais seriam dispostos os spins. A escolha mais natural é algum conjunto $\mathbb{Z}^{D}, D \in \mathbb{N}$. Sendo $\mathbb{Z}^{3}$ o conjunto mais próximo à disposição de spins eletrônicos no ambiente físico tridimensional, esse caso desperta particular interesse. Estudar a variação das características do sistema de acordo com a dimensão $D$ é um tópico essencial para entender como as interações entre os entes individuais podem gerar ordem em grande escala.

Outra questão a ser abordada ao se definir o sistema é a interação $J_{i j}$ entre os pares de spins $(i, j)$. É comum se adotar interações translacionalmente invariantes, isto é, dependentes apenas da distância entre os spins, $J_{i j}=J(|i-j|)$. Essas interações podem ser tanto de alcance finito como de alcance infinito. Diz-se uma interação ser de alcance finito $A$ se $|i-j|>A \Rightarrow J_{i j}=0$. Se não houver um limitante $A$, a interação é dita de alcance infinito.

Quando as interações são restritas aos sítios mais próximos entre si, dizemos ser uma interação de primeiros vizinhos. Nesse caso, as constantes de acoplamento são $J_{i j}=J$ se $|i-j|=1$ e $J_{i j}=0$ se $|i-j| \neq 1$. Um modelo definido com esse tipo de interação de curto alcance para a rede em $\mathbb{Z}$ foi estudado na tese de Doutorado de Ernst Ising [15]. Ising mostrou que no limite quando $N \rightarrow \infty$ não existe magnetização espontânea em nenhuma temperatura. Posteriormente, mediante o uso de uma abordagem por matriz de transferência, também deduziu-se a não existência de transição de fase para qualquer modelo em $D=1$ com interações translacionalmente invariantes e de curto alcance, para detalhes veja [1] e referências contidas. 
Já para dimensões maiores $(D \geq 2)$, uma técnica hoje conhecida como Argumento de Peierls foi pela primeira vez aplicada em [19], mostrando a existência em $D=2$ de transição de fase abaixo de um certa temperatura, mesmo para a interação de primeiros vizinhos. Veremos nos capítulos seguintes que o aumento das interações entre os spins não diminui a magnetização. Assim, a existência de transição de fase em um modelo definido em $D=2$ e com interação de primeiros vizinhos também garante a existência de transição de fase para qualquer modelo em $D=2$, desde que a interação seja não-negativa e pelo menos as interações de primeiros vizinhos sejam não-nulas.

A existência de transição de fase em $D \geq 3$ também é garantida pelo Argumento de Peierls, já que um sistema em $D=3$ pode ser visto como uma infinidade de planos paralelos com sistemas em $D=2$. Os planos paralelos independentes equivalem a fixar em zero as constantes de acoplamento entre quaisquer dois spins de planos diferentes. Mas, como os planos não interagentes têm magnetização positiva por si só, não diminuímos essa magnetização ao aumentar as constantes de acoplamento ligando spins de planos diferentes. O mesmo argumento vale para $D>3$, pois um sistema em $D+1$ pode ser visto como uma série de sistemas em $D$ acoplados entre si.

O foco desta dissertação são os modelos de Ising definidos em $D=1$, mas com interações de longo alcance. Veremos na seção 3.7 que a compreensão de tais modelos com constantes de acoplamento da forma $J_{i j}=$ $1 /|i-j|^{\alpha}$ implica na compreensão de uma vasta gama de modelos de Ising com interações translacionalmente invariantes e de longo alcance. Assim, veremos aqui praticamente todos os casos unidimensionais com interações de dois corpos ferromagnéticas, invariantes por translação cujo o decaimento segue uma lei de potência com expoente $\alpha \neq 2$.

Os caso em que $\alpha \leq 1$ são de pouco interesse, pois a soma das energias de interação de um spin com os demais diverge. Nessa situação todos os spins se alinham e temos simplesmente

$$
\lim _{h \rightarrow 0^{+}} \lim _{\Lambda \rightarrow \mathbb{Z}} m_{\Lambda, \beta, h}=+1
$$

para qualquer valor do inverso da temperatura $\beta>0$. Já o caso $\alpha>2$ é de pouco interesse pelo motivo inverso. Nesse caso,

$$
\lim _{h \rightarrow 0^{+}} \lim _{\Lambda \rightarrow \mathbb{Z}} m_{\Lambda, \beta, h}=0
$$


para qualquer $\beta>0$. Não há, assim, transição de fase se $\alpha>2$. Abordaremos esses sistemas com mais detalhes na Seção 3.7 .

Os outros modelos de Ising unidimensionais com interação translacionalmente invariante do tipo lei de potência, $J_{i j}=1 /|i-j|^{\alpha}$, podem ser divididos em outras duas classes. A primeira classe é formada pelos sistemas com $1<\alpha<2$. Esse é o tema do Capítulo 4 desta dissertação, no qual é apresentada a demonstração da existência de transição de fase a partir de um certo $\beta$, seguindo passo-a-passo a argumentação de Dyson em [5]. A segunda classe é formada pelo modelo $\operatorname{com} \alpha=2$. Neste caso temos $J_{i j}=|i-j|^{-2}$ e o modelo de Ising com tais constantes de acoplamento tem magnetização espontânea para temperaturas suficientemente baixas. A prova da existência de magnetização espontânea foi dada por Frölich e Spencer, cerca de dez anos depois da prova de Dyson para os casos $1<\alpha<2$. A exposição completa do argumento no caso limite requer técnicas distintas às que são abordadas nesta dissertação e o leitor interessado pode ver a referência [10], para maiores detalhes.

Juntando todos esses casos, temos uma classificação completa para o modelo de Ising unidimensional quanto à ausência ou não de transição de fase quando as constantes de acoplamento são da forma $J_{i j}=1 /|i-j|^{\alpha}$. 


\section{Capítulo 2}

\section{Propriedades Gerais}

\subsection{Introdução}

Este capítulo trata de algumas propriedades gerais de modelos de Ising com constantes de acoplamento $J_{i j}$ ferromagnéticas, mas sem qualquer outra restrição. Em oposição, os capítulos seguintes tratarão de modelos cujas constantes $J_{i j}$ têm certas propriedades adicionais, como a invariância translacional.

No início deste capítulo, na Seção 2.2, veremos três desigualdades de correlação e algumas consequências imediatas que serão utilizadas em várias passagens subsequentes. Em seguida, na Seção 2.3, definiremos os modelos com condições exteriores (ou condições de contorno). Nas Seções 2.4 e 2.5 utilizaremos as desigualdades da Seção 2.2 para demonstrar respectivamente a existência do limite da magnetização e uma condição suficiente para sua positividade.

Neste capítulo, apesar de tratarmos de sistemas com um número finito $|\Lambda|$ de sítios, quando calcularmos valores esperados e outras somas ponderadas com respeito a medida de probabilidade $\mathbb{P}_{\Lambda}$, utilizaremos os símbolos de integrais em $\Omega_{\Lambda}$ para representar o somatório ponderado sobre o espaço das configurações $\Omega_{\Lambda}$. Para ser mais preciso, se $\kappa: \mathcal{F}_{\Lambda} \rightarrow \mathbb{R}$ denota a medida da contagem em $\Omega_{\Lambda}$, então temos para qualquer função $f: \Omega_{\Lambda} \rightarrow \mathbb{R}$ que

$$
\langle f\rangle_{\Lambda} \equiv \sum_{\sigma \in \Omega_{\Lambda}} f(\sigma) \mathbb{P}_{\Lambda}(\sigma)=\int_{\Omega_{\Lambda}} f(\sigma) \mathbb{P}_{\Lambda}(\sigma) d \kappa(\sigma)
$$

A segunda igualdade segue imediatamente de $\Omega_{\Lambda}$ ser finito, a aplicação $\sigma \mapsto f(\sigma) \mathbb{P}_{\Lambda}(\sigma)$ ser uma função simples e da definição da integral de Lebes- 
gue de uma função simples.

Poderíamos ter optado por adotar simplesmente os somatórios, mas preferimos adequar a notação deste capítulo a outros textos onde são tomados modelos com infinitos estados (com sítios tomados em $\mathbb{Z}$, ou spins tomando valores em $\mathbb{R}$, por exemplo).

\subsection{Desigualdades de Correlação}

Nesta seção demonstraremos duas desigualdades de correlação necessárias nos passos seguintes. Também apresentaremos uma consequência imediata de cada uma delas. Essas consequências correspondem à monotonicidade da magnetização específica tanto com o aumento do campo externo $h_{i}$ em qualquer spin $i \in \Lambda$, bem como com o aumento do acoplamento entre dois spins $J_{i j}$. Veremos que para muitos sistemas, o aumento de $h_{i}$ ou $J_{i j}$ não diminui a magnetização.

A primeira dessas desigualdades é denominada Desigualdade de FKG em homenagem a Fortuin, Kasteleyn e Ginibre, veja [9]. Prosseguimos com algumas definições prévias e o enunciado da desigualdade.

Definição 2.2.1 (Ordem Parcial em $\Omega_{\Lambda}$ ). Dadas duas configurações $\omega, \sigma \in$ $\Omega_{\Lambda}$, diz-se que $\omega \preceq \sigma$ quando:

$$
\forall i \in \Lambda \text { temos que } \omega_{i} \leq \sigma_{i}
$$

Definição 2.2.2 (Função Não-Decrescente). Uma função $f: \Omega_{\Lambda} \rightarrow \mathbb{R}$ é dita não-decrescente se:

$$
\forall \omega, \sigma \in \Omega \quad \text { tal que } \omega \preceq \sigma \text { temos que } f(\omega) \leq f(\sigma) .
$$

Observação 2.2.1. Funções não-decrescentes, no sentido introduzido acima, serão também as vezes chamadas de funções crescentes.

Exemplos:

- A função $f(\sigma)=S_{\Lambda}(\sigma)$ é crescente, já que a inversão de qualquer spin de -1 para +1 aumenta o valor de $f(\sigma)$.

- Também é crescente a função $g(\sigma)=\sigma_{\alpha}$, que dá o spin no sítio $\alpha$. Perceba que a alteração de qualquer spin em sítio diferente de $\alpha$ não altera o valor de $g$ e a inversão do spin em $\alpha$ de -1 para +1 aumenta $g$ de -1 para +1 . 
- A função $h(\sigma)=\sigma_{a} \sigma_{b}$ não é crescente. Observe que se o estado $\sigma$ possui $\sigma_{a}=\sigma_{b}=-1$, temos $h(\sigma)=+1$. Porém se $\widetilde{\sigma}_{a}=+1$ e, para todo sítio $i \neq a$, tivermos $\widetilde{\sigma}_{i}=\sigma_{i}$, decorre que $\sigma \preceq \widetilde{\sigma}$, mas $+1=h(\sigma)>h(\widetilde{\sigma})=-1$.

- Pelo mesmo motivo do item anterior, não é crescente a função $S_{\Lambda}^{2}(\sigma)$.

Utilizaremos as definições apresentadas até aqui para enunciar a citada desigualdade no teorema a seguir.

Teorema 2.2.2. (Desigualdade de FKG) Sejam $f, g: \Omega_{\Lambda} \rightarrow \mathbb{R}$ duas funções crescentes, no sentido da Definição 2.2.2. Sejam $\mathbb{P}_{\Lambda}$ a medida de Gibbs do modelo de Ising dada por (1.1) com Hamiltoniano (1.2) $e\langle\cdot\rangle_{\Lambda}$ a esperança com respeito a medida $\mathbb{P}_{\Lambda}$. Se para todo $i, j \in \Lambda$ temos que $J_{i j} \geq 0$ e $h_{i} \in \mathbb{R}$. Então

$$
\langle f g\rangle_{\Lambda}-\langle f\rangle_{\Lambda}\langle g\rangle_{\Lambda} \geq 0 .
$$

Demonstração. A demonstração será por indução no comprimento da caixa, $|\Lambda|$. A primeira tarefa será reorganizar o lado esquerdo da desigualdade (2.2). Usando a definição do valor esperado e (2.1) temos

$$
\langle f g\rangle_{\Lambda}-\langle f\rangle_{\Lambda}\langle g\rangle_{\Lambda}=\int_{\Omega_{\Lambda}} f(\sigma) g(\sigma) \mathbb{P}_{\Lambda}(\sigma) d \kappa(\sigma)-\int_{\Omega_{\Lambda}} f(\sigma) \mathbb{P}_{\Lambda}(\sigma) d \kappa(\sigma) \cdot \int_{\Omega_{\Lambda}} g(\widetilde{\sigma}) \mathbb{P}_{\Lambda}(\widetilde{\sigma}) d \kappa(\widetilde{\sigma}) .
$$

Como as integrais acima são simplesmente somas finitas e temos que $\int_{\Omega_{\Lambda}} \mathbb{P}_{\Lambda}(\sigma) d \kappa(\sigma)=1$, podemos reescrever o lado direito da expressão acima como a seguinte integral dupla

$$
\int_{\Omega_{\Lambda}} \int_{\Omega_{\Lambda}}\left[(f(\sigma) g(\sigma)-f(\sigma) g(\widetilde{\sigma})) \mathbb{P}_{\Lambda}(\sigma) \mathbb{P}_{\Lambda}(\widetilde{\sigma})\right] d \kappa(\sigma) d \kappa(\widetilde{\sigma}) .
$$

Já que $\sigma$ e $\widetilde{\sigma}$ variam sobre o mesmo espaço $\Omega_{\Lambda}$, podemos trocar $\sigma$ por $\widetilde{\sigma}$ e vice-versa sem alterar a integral. Sendo assim, a integral anterior equivale à seguinte.

$$
\int_{\Omega_{\Lambda}} \int_{\Omega_{\Lambda}}\left[(f(\widetilde{\sigma}) g(\widetilde{\sigma})-f(\widetilde{\sigma}) g(\sigma)) \mathbb{P}_{\Lambda}(\sigma) \mathbb{P}_{\Lambda}(\widetilde{\sigma})\right] d \kappa(\sigma) d \kappa(\widetilde{\sigma}) .
$$

Somando as duas últimas expressões e multiplicando por $1 / 2$, retomamos o que tínhamos inicialmente, pois ambas as expressões têm o mesmo valor. Portanto a integral dupla acima pode ser reescrita como

$$
\frac{1}{2} \int_{\Omega_{\Lambda}} \int_{\Omega_{\Lambda}}\left[(f(\sigma) g(\sigma)-f(\sigma) g(\widetilde{\sigma})+f(\widetilde{\sigma}) g(\widetilde{\sigma})-f(\widetilde{\sigma}) g(\sigma)) \mathbb{P}_{\Lambda}(\sigma) \mathbb{P}_{\Lambda}(\widetilde{\sigma})\right] d \kappa(\sigma) d \kappa(\widetilde{\sigma}) .
$$


Fatorando a expressão em parêntesis no integrando, ficamos com

$$
\frac{1}{2} \int_{\Omega_{\Lambda}} \int_{\Omega_{\Lambda}}\left[(f(\sigma)-f(\widetilde{\sigma}))(g(\sigma)-g(\widetilde{\sigma})) \mathbb{P}_{\Lambda}(\sigma) \mathbb{P}_{\Lambda}(\widetilde{\sigma})\right] d \kappa(\sigma) d \kappa(\widetilde{\sigma}) .
$$

Podemos agora analisar a expressão acima nos restringindo ao caso $|\Lambda|=1$, isto é, $f$ e $g$ funções do spin de apenas um (e do mesmo) sítio. Sem perda de generalidade, suponhamos que esse sítio cujo spin dependem as funções $f$ e $g$ seja o de coordenada 0 . Dados dois estados $\sigma$ e $\widetilde{\sigma}$ tais que $\sigma_{0} \leq \widetilde{\sigma}_{0}$, temos que $f(\sigma)-f(\widetilde{\sigma}) \leq 0$ e $g(\sigma)-g(\widetilde{\sigma}) \leq 0$, pois $f$ e $g$ são funções crescentes e dependem apenas do spin de coordenada 0. Logo, nesse caso, o integrando que aparece acima é não-negativo. Similarmente, se $\sigma_{0} \geq \widetilde{\sigma}_{0}$, temos $f(\sigma)-f(\widetilde{\sigma}) \geq 0$ e $f(\sigma)-g(\widetilde{\sigma}) \geq 0$. Novamente o integrando acima é não-negativo e em ambos os casos concluímos que a integral acima é sempre não-negativa.

Dessa observação, segue que a Desigualdade de FKG (2.2) é verdadeira para o caso $|\Lambda|=1$, independente da medida de probabilidade $\mathbb{P}_{\Lambda}$. Em particular, a desigualdade é verdadeira para medidas de Gibbs de modelos de Ising ferromagnético.

Vamos supor agora que o teorema é verdadeiro para um subconjunto arbitrário $\Lambda \subset \mathbb{Z}$ de cardinalidade $|\Lambda| \leq n$.

Considere agora $\Lambda \subset \mathbb{Z}$ tal que $|\Lambda|=n+1$. Pela equação (2.1) e definição de $\mathbb{P}_{\Lambda}$ temos que

$$
\langle f g\rangle_{\Lambda}=\int_{\Omega_{\Lambda}} f(\sigma) g(\sigma) \frac{\exp \left(-\beta H_{\Lambda}(\sigma)\right)}{Z_{\Lambda}} d \kappa(\sigma) .
$$

Para utilizar a hipótese de indução, a ideia é fixar um dos spins, em algum sítio $\alpha \in \Lambda$. Feita uma escolha de $\alpha \in \Lambda$ defina $\Lambda^{\prime}=\Lambda \backslash\{\alpha\}$. Note que podemos reescrever o hamiltoniano $H_{\Lambda}$ isolando as interações com o spin 
escolhido, da seguinte forma:

$$
\begin{aligned}
H_{\Lambda}(\sigma) & =-\sum_{i \in \Lambda}\left\{\sum_{j \in \Lambda} \frac{1}{2} J_{i j} \sigma_{i} \sigma_{j}+h_{i} \sigma_{i}\right\} \\
& =-\sum_{i \in \Lambda^{\prime}}\left\{\sum_{j \in \Lambda^{\prime}} \frac{1}{2} J_{i j} \sigma_{i} \sigma_{j}+h_{i} \sigma_{i}\right\}-\sum_{i \in \Lambda^{\prime}} J_{i \alpha} \sigma_{\alpha} \sigma_{i}-h_{\alpha} \sigma_{\alpha} \\
& =-\sum_{i \in \Lambda^{\prime}}\left\{\sum_{j \in \Lambda^{\prime}} \frac{1}{2} J_{i j} \sigma_{i} \sigma_{j}+\left(h_{i}+J_{i \alpha} \sigma_{\alpha}\right) \sigma_{i}\right\}-h_{\alpha} \sigma_{\alpha} \\
& =-\sum_{i \in \Lambda^{\prime}}\left\{\sum_{j \in \Lambda^{\prime}} \frac{1}{2} J_{i j} \sigma_{i} \sigma_{j}+h_{i}^{\prime}\left(\sigma_{\alpha}\right) \sigma_{i}\right\}-h_{\alpha} \sigma_{\alpha},
\end{aligned}
$$

onde $h_{i}^{\prime}\left(\sigma_{\alpha}\right) \equiv h_{i}+J_{i \alpha} \sigma_{\alpha}$. É perceptível na equação acima que, se fixarmos $\sigma_{\alpha}$, podemos definir um novo modelo de Ising no volume $\Lambda^{\prime}$ (que tem cardinalidade $\left|\Lambda^{\prime}\right|=n$ ) cujo Hamiltoniano é dado pelo lado direito da expressão acima $\operatorname{com} h_{i}^{\prime}\left(\sigma_{\alpha}\right)=h_{i}+J_{i \alpha} \sigma_{\alpha}$.

A expressão acima na verdade nos fornece uma maneira de definir dois modelos de Ising em $\Lambda^{\prime}$, um com $\sigma_{\alpha}=+1$ e outro com $\sigma_{\alpha}=-1$. Fixado o valor de $\sigma_{\alpha}$ a medida de Gibbs desse novo sistema é definida pelo Hamiltoniano abaixo,

$$
H_{\Lambda^{\prime}, \sigma_{\alpha}}=-\sum_{i \in \Lambda^{\prime}}\left\{\sum_{j \in \Lambda^{\prime}} \frac{1}{2} J_{i j} \sigma_{i} \sigma_{j}+\left(h_{i}+J_{i \alpha} \sigma_{\alpha}\right) \sigma_{i}\right\}-h_{\alpha} \sigma_{\alpha} .
$$

Pela hipótese de indução para qualquer uma das duas escolha de $\sigma_{\alpha}$ temos

$$
\langle f g\rangle_{\Lambda^{\prime}}-\langle f\rangle_{\Lambda^{\prime}}\langle g\rangle_{\Lambda^{\prime}} \geq 0
$$

Agora voltamos nossa atenção para (2.3), no intuito decompor esta integral em duas partes, uma dependendo apenas do spin $\alpha$, a outra em $\Lambda^{\prime}$, onde será possível aplicar a hipótese de indução. Primeiro observamos que

$$
\begin{aligned}
\langle f g\rangle_{\Lambda} & =\int_{\Omega_{\Lambda}} f(\sigma) g(\sigma) \frac{\exp \left(-\beta H_{\Lambda}(\sigma)\right)}{Z_{\Lambda}} d \kappa(\sigma) \\
& =\int_{\{-1,+1\}}\left[\int_{\Omega_{\Lambda^{\prime}}} f\left(\sigma^{\prime}, \sigma_{\alpha}\right) g\left(\sigma^{\prime}, \sigma_{\alpha}\right) \frac{\exp \left(-\beta H_{\Lambda^{\prime}, \sigma_{\alpha}}\left(\sigma^{\prime}, \sigma_{\alpha}\right)\right)}{Z_{\Lambda}} d \kappa\left(\sigma^{\prime}\right)\right] d \kappa\left(\sigma_{\alpha}\right),
\end{aligned}
$$

onde na segunda igualdade abusamos da notação já que a rigor $\kappa$ é a medida de contagem em $\Omega_{\Lambda}$ e acima usamos $\kappa$ para denotar tanto a medida 
da contagem em $\Omega_{\Lambda^{\prime}}$ quanto a medidada de contagem em $\Omega_{\{\alpha\}}=\{-1,1\}$. Observando que $Z_{\Lambda^{\prime}, \sigma_{\alpha}} \neq 0$, podemos multiplicar a dividir o integrando por esse valor para reescrever o lado direito da igualdade acima como

$$
\int_{\{-1,+1\}}\left[\int_{\Omega_{\Lambda^{\prime}}} f\left(\sigma^{\prime}, \sigma_{\alpha}\right) g\left(\sigma^{\prime}, \sigma_{\alpha}\right) \frac{\exp \left(-\beta H_{\Lambda^{\prime}, \sigma_{\alpha}}\left(\sigma^{\prime}, \sigma_{\alpha}\right)\right)}{Z_{\Lambda^{\prime}, \sigma_{\alpha}}} d \kappa\left(\sigma^{\prime}\right)\right] \cdot \frac{Z_{\Lambda^{\prime}, \sigma_{\alpha}}}{Z_{\Lambda}} d \kappa\left(\sigma_{\alpha}\right) .
$$

Note que a expressão em colchetes é por definição $\langle f g\rangle_{\Lambda^{\prime}, \sigma_{\alpha}}$ e portanto a integral acima pode ser reescrita como

$$
\int_{\{-1,+1\}}\langle f g\rangle_{\Lambda^{\prime}, \sigma_{\alpha}} \cdot \frac{Z_{\Lambda^{\prime}, \sigma_{\alpha}}}{Z_{\Lambda}} d \kappa\left(\sigma_{\alpha}\right) .
$$

Aplicando a hipótese de indução obtemos a seguinte desigualdade

$$
\int_{\{-1,+1\}}\langle f g\rangle_{\Lambda^{\prime}, \sigma_{\alpha}} \cdot \frac{Z_{\Lambda^{\prime}, \sigma_{\alpha}}}{Z_{\Lambda}} d \kappa\left(\sigma_{\alpha}\right) \leq \int_{\{-1,+1\}}\langle f\rangle_{\Lambda^{\prime}, \sigma_{\alpha}}\langle g\rangle_{\Lambda^{\prime}, \sigma_{\alpha}} \cdot \frac{Z_{\Lambda^{\prime}, \sigma_{\alpha}}}{Z_{\Lambda}} d \kappa\left(\sigma_{\alpha}\right) .
$$

Se $\gamma\left(\sigma_{\alpha}\right)=\langle f\rangle_{\Lambda^{\prime}, \sigma_{\alpha}}$ e $\eta\left(\sigma_{\alpha}\right)=\langle g\rangle_{\Lambda^{\prime}, \sigma_{\alpha}}$ fossem funções não-decrescente, então poderíamos usar a desigualdade provada para o caso $|\Lambda|=1$ (que era independente da medida de probabilidade) para concluir que

$$
\begin{aligned}
\langle f g\rangle_{\Lambda} & \leq \int_{\{-1,+1\}}\langle f\rangle_{\Lambda^{\prime}, \sigma_{\alpha}}\langle g\rangle_{\Lambda^{\prime}, \sigma_{\alpha}} \cdot \frac{Z_{\Lambda^{\prime}, \sigma_{\alpha}}}{Z_{\Lambda}} d \kappa\left(\sigma_{\alpha}\right) \\
& \leq \int_{\{-1,+1\}}\langle f\rangle_{\Lambda^{\prime}, \sigma_{\alpha}} \cdot \frac{Z_{\Lambda^{\prime}, \sigma_{\alpha}}}{Z_{\Lambda}} \rho\left(d \sigma_{\alpha}\right) \cdot \int_{\{-1,+1\}}\langle g\rangle_{\Lambda^{\prime}, \sigma_{\alpha}} \cdot \frac{Z_{\Lambda^{\prime}, \sigma_{\alpha}}}{Z_{\Lambda}} d \kappa\left(\sigma_{\alpha}\right) \\
& =\langle f\rangle_{\Lambda}\langle g\rangle_{\Lambda}
\end{aligned}
$$

onde usamos que

$$
\int_{\{-1,+1\}} \frac{Z_{\Lambda^{\prime}, \sigma_{\alpha}}}{Z_{\Lambda}} d \kappa\left(\sigma_{\alpha}\right)=1 \text {, ou equivalentemente } \frac{Z_{\Lambda^{\prime},+1}+Z_{\Lambda^{\prime},-1}}{Z_{\Lambda}}=1 .
$$

Resta-nos, pois, provar que a função $\gamma\left(\sigma_{\alpha}\right)$ é crescente, pois argumento análogo também prova que $\eta\left(\sigma_{\alpha}\right)$ é crescente. Isso equivale a mostrar que $\gamma(+1) \geq \gamma(-1)$, pois $\gamma$ é função de apenas 1 spin.

Denote por $\mathbb{P}_{\Lambda^{\prime}, \sigma_{\alpha}}$ a medida de Gibbs em $\Omega_{\Lambda^{\prime}}$ correspondente ao hamiltoniano $H_{\Lambda^{\prime}, \sigma_{\alpha}}$. Próximo passo é mostrar as duas desigualdades abaixo, concluindo por fim que $\gamma$ é crescente e com isso, a demonstração da desi- 
gualdade de FKG.

$$
\begin{aligned}
\gamma(+1) & =\langle f\rangle_{\Lambda^{\prime},+1} \\
& =\int_{\Omega_{\Lambda^{\prime}}} f\left(\sigma^{\prime},+1\right) \mathbb{P}_{\Lambda^{\prime},+1}\left(\sigma^{\prime}\right) d \kappa\left(\sigma^{\prime}\right) \\
& \stackrel{(*)}{\geq} \int_{\Omega_{\Lambda^{\prime}}} f\left(\sigma^{\prime},-1\right) \mathbb{P}_{\Lambda^{\prime},+1}\left(\sigma^{\prime}\right) d \kappa\left(\sigma^{\prime}\right) \\
& \stackrel{(* *)}{\geq} \int_{\Omega_{\Lambda^{\prime}}} f\left(\sigma^{\prime},-1\right) \mathbb{P}_{\Lambda^{\prime},-1}\left(\sigma^{\prime}\right) d \kappa\left(\sigma^{\prime}\right) \\
& =\langle f\rangle_{\Lambda^{\prime},-1} \\
& =\gamma(-1) .
\end{aligned}
$$

A primeira das desigualdades, marcada com $(*)$, é trivialmente verdadeira, pois $f$ é uma função não decrescente, logo $f\left(\sigma^{\prime},+1\right) \geq f\left(\sigma^{\prime},-1\right)$. Para obter a segunda desigualdade, marcada com $(* *)$, será preciso uma análise um pouco mais elaborada.

Para cada $\sigma^{\prime} \in \Omega_{\Lambda^{\prime}}$ fixado, a aplicação $\sigma_{\alpha} \mapsto \mathbb{P}_{\Lambda^{\prime}, \sigma_{\alpha}}\left(\sigma^{\prime}\right)$ tem como domínio o conjunto $\{-1,+1\}$. De maneira natural podemos estender o domínio desta aplicação para o intervalo fechado $[-1,+1]$. Bastando para isto estender o Hamiltoniano da seguinte forma

$$
H_{\Lambda^{\prime}, t}=-\sum_{i \in \Lambda^{\prime}}\left\{\sum_{j \in \Lambda^{\prime}} \frac{1}{2} J_{i j} \sigma_{i} \sigma_{j}+\left(h_{i}+J_{i \alpha} t\right) \sigma_{i}\right\}-h_{\alpha} t
$$

e considerar a medida de Gibbs dada como em (1.1) de forma que a probabilidade de uma configuração $\sigma^{\prime}$ seja dada agora por

$$
\mathbb{P}_{\Lambda, t}\left(\sigma^{\prime}\right)=\frac{\exp \left[-\beta H_{\Lambda^{\prime}, t}\left(\sigma^{\prime}\right)\right]}{Z_{\Lambda, t}}, \quad \text { onde } Z_{\Lambda, t}=\sum_{\omega \in \Omega_{\Lambda^{\prime}}} \exp \left[-\beta H_{\Lambda, t}(\omega)\right]
$$

Esta observação nos motiva a considerar a seguinte função

$$
F(t)=\int_{\Omega_{\Lambda^{\prime}}} f\left(\sigma^{\prime},-1\right) \mathbb{P}_{\Lambda^{\prime}, t}\left(\sigma^{\prime}\right) d \kappa\left(\sigma^{\prime}\right)
$$

Evidentemente, $F(-1)=\gamma(-1)$ e $F(+1)=\gamma(+1)$. Vamos mostrar que a derivada de $F$ é positiva e disso seguirá a desigualdade $(* *)$.

Lembrando que a integral que define $F$ é na verdade uma soma finita 
temos que

$$
\begin{aligned}
\frac{d}{d t} F(t) & =\frac{d}{d t} \int_{\Omega_{\Lambda^{\prime}}}\left[f\left(\sigma^{\prime},-1\right) \mathbb{P}_{\Lambda^{\prime}, t}\left(\sigma^{\prime}\right)\right] d \kappa\left(\sigma^{\prime}\right) \\
& =\int_{\Omega_{\Lambda^{\prime}}}\left[f\left(\sigma^{\prime},-1\right) \frac{d}{d t} \mathbb{P}_{\Lambda^{\prime}, t}\left(\sigma^{\prime}\right)\right] d \kappa\left(\sigma^{\prime}\right) .
\end{aligned}
$$

Resta calcular $\frac{d}{d t} \mathbb{P}_{\Lambda^{\prime}, t}\left(\sigma^{\prime}\right)$ para cada $\sigma^{\prime} \in \Omega_{\Lambda^{\prime}}$ fixado. Usando definição de $\mathbb{P}_{\Lambda^{\prime}, t}\left(\sigma^{\prime}\right)$ temos:

$$
\begin{aligned}
\frac{d}{d t} \mathbb{P}_{\Lambda^{\prime}, t}\left(\sigma^{\prime}\right) & =\frac{d}{d t} \frac{\exp \left(-\beta H_{\Lambda^{\prime}, t}\left(\sigma^{\prime}\right)\right)}{Z_{\Lambda^{\prime}, t}} \\
& =\frac{Z_{\Lambda^{\prime}, t} \frac{d}{d t} \exp \left(-\beta H_{\Lambda^{\prime}, t}\left(\sigma^{\prime}\right)\right)-\exp \left(-\beta H_{\Lambda^{\prime}, t}\left(\sigma^{\prime}\right)\right) \frac{d}{d t} Z_{\Lambda^{\prime}, t}}{\left(Z_{\Lambda^{\prime}, t}\right)^{2}} \\
& =-\beta \frac{\exp \left(-\beta H_{\Lambda^{\prime}, t}\left(\sigma^{\prime}\right)\right) \frac{d}{d t} H_{\Lambda^{\prime}, t}\left(\sigma^{\prime}\right)-\exp \left(-\beta H_{\Lambda^{\prime}, t}\left(\sigma^{\prime}\right)\right)\left\langle\frac{d}{d t} H_{\Lambda^{\prime}, t}\right\rangle_{\Lambda^{\prime}, t}}{Z_{\Lambda^{\prime}, t}} \\
& =-\beta \frac{\exp \left(-\beta H_{\Lambda^{\prime}, t}\left(\sigma^{\prime}\right)\right)}{Z_{\Lambda^{\prime}, t}}\left[\frac{d}{d t} H_{\Lambda^{\prime}, t}\left(\sigma^{\prime}\right)-\left\langle\frac{d}{d t} H_{\Lambda^{\prime}, t}\right\rangle_{\Lambda^{\prime}, t}\right] \\
& =-\beta \mathbb{P}_{\Lambda^{\prime}, t}\left(\sigma^{\prime}\right)\left[\frac{d}{d t} H_{\Lambda^{\prime}, t}\left(\sigma^{\prime}\right)-\left\langle\frac{d}{d t} H_{\Lambda^{\prime}, t}\right\rangle_{\Lambda^{\prime}, t}\right]
\end{aligned}
$$

Usando esta igualdade em (2.7) ficamos com

$$
\begin{aligned}
\frac{d}{d t} F(t) & =\frac{d}{d t} \int_{\Omega_{\Lambda^{\prime}}} f\left(\sigma^{\prime},-1\right) \mathbb{P}_{\Lambda^{\prime}, t}\left(\sigma^{\prime}\right) d \kappa\left(\sigma^{\prime}\right) \\
& =-\beta \int_{\Omega_{\Lambda^{\prime}}}\left(f\left(\sigma^{\prime},-1\right)\left[\frac{d}{d t} H_{\Lambda^{\prime}, t}\left(\sigma^{\prime}\right)-\left\langle\frac{d}{d t} H_{\Lambda^{\prime}, t}\right\rangle_{\Lambda^{\prime}, t}\right] \mathbb{P}_{\Lambda^{\prime}, t}\left(\sigma^{\prime}\right)\right) d \kappa\left(\sigma^{\prime}\right) \\
& =\beta\left[\left\langle\left(-\frac{d}{d t} H_{\Lambda^{\prime}, t}\right) f(\cdot,-1)\right\rangle_{\Lambda^{\prime}, t}-\left\langle-\frac{d}{d t} H_{\Lambda^{\prime}, t}\right\rangle_{\Lambda^{\prime}, t}\langle f(\cdot,-1)\rangle_{\Lambda^{\prime}, t}\right] .
\end{aligned}
$$

Observe que a expressão entre colchetes na última linha é exatamente um dos lados da Desigualdade de FKG aplicada às funções $-\frac{d}{d t} H_{\Lambda^{\prime}, t}$ e $f(\cdot,-1)$. Como $f$ é crescente a função $\sigma^{\prime} \mapsto f\left(\sigma^{\prime},-1\right)$ também é crescente. Por (2.5) temos que

$$
-\frac{d}{d t} H_{\Lambda^{\prime}, t}\left(\sigma^{\prime}\right)=\sum_{i \in \Lambda^{\prime}} \frac{J_{i \alpha}}{2} \sigma_{i}+h_{\alpha} .
$$


Daí é fácil ver que a função $-\frac{d}{d t} H_{\Lambda^{\prime}, t}$ é crescente. Finalmente podemos voltar a (2.8) e usar a hipótese de indução para deduzir que $\frac{d}{d t} F(t) \geq 0$. Isso conclui a demonstração da desigualdade de FKG.

A desigualdade acima demonstrada pode ser usada para esclarecer a relação entre o campo externo e a magnetização. É de se esperar que a magnetização e também a magnetização específica não decresça com qualquer aumento no campo externo. Assim, para um aumento no campo, esperamos numa situação extrema, que magnetização ou a magnetização específica fique constante e, nos outros casos, que elas aumentem. Para mostrar que esse fato é verdadeiro vamos usar a Desigualdade de FKG (2.2). Como os cálculos são absolutamente semelhantes vamos verificar esse fato para o caso da magnetização específica. A ideia é calcular as derivadas parciais da magnetização específica com respeito a cada coordenada do campo externo $\left\{h_{i}\right\}_{i \in \Lambda}$ e mostrar que elas são não-negativas. Para facilitar a notação, no segue vamos escrever simplesmente $m_{\Lambda, \beta}$ para denotar $m_{\Lambda, \beta,\left\{h_{i}\right\}_{i \in \Lambda}}$. Assim, fixado $i \in[-N, N] \cap \mathbb{Z}$ temos que

$$
\frac{\partial}{\partial h_{i}} m_{\Lambda, \beta}=\frac{\partial}{\partial h_{i}} \int_{\Omega_{\Lambda}} \frac{\sum_{j \in \Lambda} \sigma_{j}}{|\Lambda|} \mathbb{P}_{\Lambda}(\sigma) d \kappa(\sigma)=|\Lambda|^{-1} \int_{\Omega_{\Lambda}} \sum_{j \in \Lambda} \sigma_{j} \frac{\partial}{\partial h_{i}} \mathbb{P}_{\Lambda}(\sigma) d \kappa(\sigma) .
$$

Antes de prosseguir, vamos calcular $\partial \mathbb{P}_{\Lambda}(\sigma) / \partial h_{i}$.

$$
\begin{aligned}
\frac{\partial \mathbb{P}_{\Lambda}(\sigma)}{\partial h_{i}} & =\frac{\partial}{\partial h_{i}}\left[\frac{\exp \left(-\beta H_{\Lambda}(\sigma)\right)}{Z_{\Lambda}}\right]=\frac{Z_{\Lambda} \frac{\partial}{\partial h_{i}} \exp \left(-\beta H_{\Lambda}(\sigma)\right)-\exp \left(-\beta H_{\Lambda}(\sigma)\right) \frac{\partial}{\partial h_{i}} Z_{\Lambda}}{\left(Z_{\Lambda}\right)^{2}} \\
& =\frac{\beta \exp \left(-\beta H_{\Lambda}(\sigma)\right) \frac{\partial H_{\Lambda}(\sigma)}{\partial h_{i}}+\beta \exp \left(-\beta H_{\Lambda}(\sigma)\right)\left\langle\sigma_{i}\right\rangle_{\Lambda}}{Z_{\Lambda}} \\
& =\frac{\beta \exp \left(-\beta H_{\Lambda}(\sigma)\right)}{Z_{\Lambda}}\left[\frac{\partial H_{\Lambda}(\sigma)}{\partial h_{i}}+\left\langle\sigma_{i}\right\rangle_{\Lambda}\right] \\
& =\beta \mathbb{P}_{\Lambda}(\sigma)\left[\sigma_{i}-\left\langle\sigma_{i}\right\rangle_{\Lambda}\right] .
\end{aligned}
$$


Substituindo esta expressão em (2.9) ficamos com

$$
\begin{aligned}
\frac{\partial}{\partial h_{i}} m_{\Lambda, \beta} & =|\Lambda|^{-1} \int_{\Omega_{\Lambda}} \sum_{j \in \Lambda} \sigma_{j} \frac{\partial}{\partial h_{i}} \mathbb{P}_{\Lambda}(\sigma) d \kappa(\sigma) \\
& =|\Lambda|^{-1} \beta \int_{\Omega_{\Lambda}} \sum_{j \in \Lambda} \sigma_{j} \mathbb{P}_{\Lambda}(\sigma)\left[\sigma_{i}-\left\langle\sigma_{i}\right\rangle_{\Lambda}\right] d \kappa(\sigma) \\
& =|\Lambda|^{-1} \beta \sum_{j \in \Lambda} \int_{\Omega_{\Lambda}} \sigma_{j}\left[\sigma_{i}-\left\langle\sigma_{i}\right\rangle_{\Lambda}\right] \mathbb{P}_{\Lambda}(\sigma) d \kappa(\sigma) \\
& =|\Lambda|^{-1} \beta \sum_{j \in \Lambda}\left[\int_{\Omega_{\Lambda}} \sigma_{j} \sigma_{i} \mathbb{P}_{\Lambda}(\sigma) d \kappa(\sigma)-\int_{\Omega_{\Lambda}} \sigma_{j}\left\langle\sigma_{i}\right\rangle_{\Lambda} \mathbb{P}_{\Lambda}(\sigma) d \kappa(\sigma)\right] \\
& =|\Lambda|^{-1} \beta \sum_{j \in \Lambda}\left[\left\langle\sigma_{j} \sigma_{i}\right\rangle_{\Lambda}-\left\langle\sigma_{j}\right\rangle_{\Lambda}\left\langle\sigma_{i}\right\rangle_{\Lambda}\right]
\end{aligned}
$$

Aplicando na última igualdade acima, em cada parcela do somatório, a Desigualdade de FKG com $f(\sigma)=\sigma_{i}$ e $g(\sigma)=\sigma_{j}$ temos finalmente que $\partial m_{\Lambda, \beta} / \partial h_{i} \geq 0$. Enunciamos logo abaixo esse fato como um corolário para posterior referência.

Corolário 2.2.3. No modelo de Ising ferromagnético a volume finito $\Lambda$, temos

$$
\frac{\partial}{\partial h_{i}} m_{\Lambda, \beta,\left\{h_{i}\right\}_{i \in \Lambda}}=\frac{1}{|\Lambda|} \beta \sum_{j \in \Lambda}\left[\left\langle\sigma_{j} \sigma_{i}\right\rangle_{\Lambda, \beta,\left\{h_{i}\right\}_{i \in \Lambda}}-\left\langle\sigma_{j}\right\rangle_{\Lambda, \beta,\left\{h_{i}\right\}_{i} \in \Lambda}\left\langle\sigma_{i}\right\rangle_{\Lambda, \beta,\left\{h_{i}\right\}_{i \in \Lambda}}\right] \geq 0 .
$$

Em particular, a magnetização específica não decresce com o aumento do campo externo em qualquer dos sítios de $\Lambda$.

Outra desigualdade de fundamental importância neste texto é a Desigualdade GKS-2. Essa desigualdade foi provada primeiramente por Griffiths para a correlação de pares de spins em [13]. Sua forma mais geral foi obtida por Kelly e Sherman em [16]. Devido às contribuições desses autores, a desigualdade é assim denominada. A exposição adiante toma como base a apresentação de [4] a respeito do tema.

Antes de enunciar a desigualdade com precisão, precisamos introduzir algumas notações.

Definição 2.2.3. Dado um subconjunto finito e não vazio $A \subseteq \Lambda$, a função $\sigma_{A}: \Omega_{\Lambda} \rightarrow\{-1,+1\}$ é definida por

$$
\sigma_{A}(\sigma)=\prod_{i \in A} \sigma_{i}
$$


Agora estamos prontos para enunciar precisamente a Desigualdade GKS-2.

Teorema 2.2.4 (Desigualdade de GKS-2). Seja $\Lambda \subset \mathbb{Z}$ finito e considere o modelo de Ising no volume $\Lambda$ com Hamiltoniano

$$
H_{\Lambda}(\omega)=-\sum_{i \in \Lambda}\left\{\sum_{j \in \Lambda} \frac{1}{2} J_{i j} \omega_{i} \omega_{j}+h_{i} \omega_{i}\right\}
$$

Se $J_{i, j} \geq 0$ e $h_{i} \geq 0$ para todo $i, j \in \Lambda$, então para quaisquer $A$ e $B$ subconjuntos não vazios de $\Lambda$ temos

$$
\left\langle\sigma_{A} \sigma_{B}\right\rangle_{\Lambda}-\left\langle\sigma_{A}\right\rangle_{\Lambda}\left\langle\sigma_{B}\right\rangle_{\Lambda} \geq 0
$$

Demonstração. Para esta demonstração, precisaremos criar um novo sistema definido em $\Omega_{\Lambda} \times \Omega_{\Lambda}$ correspondendo a duas cópias independentes do sistema original em $\Omega_{\Lambda}$. Para que esses sistemas sejam independentes independentes, a energia de um estado $(\omega, \widetilde{\omega}) \in \Omega_{\Lambda} \times \Omega_{\Lambda}$ será definida como a soma das energias dos estados, isto é,

$$
H_{2}(\omega, \widetilde{\omega})=H_{\Lambda}(\omega)+H_{\Lambda}(\widetilde{\omega})
$$

Analogamente, denotaremos por $\omega_{A}, \widetilde{\omega}_{A}$ as duas funções $\omega_{A}, \widetilde{\omega}_{A}: \Omega_{\Lambda}^{2}=\Omega_{\Lambda} \times$ $\Omega_{\Lambda} \rightarrow\{-1,+1\}$ tais que $\omega_{A}(\omega, \widetilde{\omega})=\sigma_{A}(\omega)$ e $\widetilde{\omega}_{A}(\omega, \widetilde{\omega})=\sigma_{A}(\widetilde{\omega})$. Também denotaremos por $\langle\cdot\rangle_{2}$ a esperança com relação à distribuição de probabilidades definida pelo hamiltoniano $H_{2}$. Podemos então reescrever o lado esquerdo de (2.12) em termos de esperanças em $\Omega_{\Lambda}^{2}$.

$$
\left\langle\sigma_{A} \sigma_{B}\right\rangle_{\Lambda}-\left\langle\sigma_{A}\right\rangle_{\Lambda}\left\langle\sigma_{B}\right\rangle_{\Lambda}=\left\langle\omega_{A} \omega_{B}\right\rangle_{2}-\left\langle\omega_{A}\right\rangle_{2}\left\langle\widetilde{\omega}_{B}\right\rangle_{2}=\left\langle\omega_{A} \omega_{B}-\omega_{A} \widetilde{\omega}_{B}\right\rangle_{2}
$$

A primeira igualdade vem do fato que, pela definição de $\omega_{A},\left\langle\omega_{A}\right\rangle_{2}=$ $\left\langle\sigma_{A}\right\rangle_{\Lambda}$. A segunda igualdade decorre da independência de $\omega_{A}$ e $\omega_{B}$, assim $\left\langle\omega_{A} \widetilde{\omega}_{B}\right\rangle_{2}=\left\langle\omega_{A}\right\rangle_{2}\left\langle\widetilde{\omega}_{B}\right\rangle_{2}$.

Definindo para cada $i \in \Lambda$ as funções $X_{i}, Y_{i}: \Omega_{\Lambda} \times \Omega_{\Lambda} \rightarrow\{-1,0,1\}$ por

$$
X_{i}(\omega, \widetilde{\omega})=\frac{\omega_{i}-\widetilde{\omega}_{i}}{\sqrt{2}} \quad \text { e } \quad Y_{i}(\omega, \widetilde{\omega})=\frac{\omega_{i}+\widetilde{\omega}_{i}}{\sqrt{2}}
$$

retomando a definição de $\sigma_{A}$ em (2.11), e usando a identidade (2.13) é possível reescrever a expressão acima em termos dessas funções, como 
segue

$$
\begin{aligned}
\left\langle\sigma_{A} \sigma_{B}\right\rangle_{\Lambda}-\left\langle\sigma_{A}\right\rangle_{\Lambda}\left\langle\sigma_{B}\right\rangle_{\Lambda} & =\left\langle\omega_{A}\left[\omega_{B}-\widetilde{\omega}_{B}\right]\right\rangle_{2} \\
& =\left\langle\prod_{i \in A} \frac{X_{i}+Y_{i}}{\sqrt{2}}\left[\prod_{i \in B} \frac{X_{i}+Y_{i}}{\sqrt{2}}-\prod_{i \in B} \frac{X_{i}-Y_{i}}{\sqrt{2}}\right]\right\rangle_{2}
\end{aligned}
$$

Quando expandimos os produtórios entre colchetes, todo termo com coeficiente negativo da expansão do produtório à direita terá um equivalente positivo no produtório à esquerda. Esses termos com coeficientes negativos serão todos cancelados, restando apenas termos positivos de produtórios de $X_{i}$ e $Y_{j}$, $i$ e $j$ variando sobre alguns subconjuntos de B.

A expansão do outro produtório, aquele fora do colchete, também trará termos em $X_{i}$ e $Y_{j}$ com coeficientes positivos (iguais a 1). Assim, ao expandir toda a expressão acima, teremos uma soma de termos da forma

$$
\left\langle\prod_{i \in C} X_{i} \prod_{j \in D} Y_{j}\right\rangle_{2}
$$

onde $C$ e $D$ são subconjuntos de $\Lambda$.

Assim, basta provar que um termo com o formato acima é necessariamente não negativo para concluir a demonstração da desigualdade (2.12). Para facilitar a notação de maneira análoga

$$
X_{A}=\prod_{i \in A} X_{i} \quad \text { e } \quad Y_{B}=\prod_{i \in B} Y_{i}
$$

Ficamos por demonstrar que $\left\langle X_{A} Y_{B}\right\rangle_{2} \geq 0$. Para tal, invocamos sua definição

$$
\left\langle X_{A} Y_{B}\right\rangle_{2}=\sum_{\omega, \widetilde{\omega} \in \Omega_{\Lambda}} X_{A}(\omega, \widetilde{\omega}) Y_{B}(\omega, \widetilde{\omega}) \frac{\exp \left[-\beta H_{2}(\omega, \widetilde{\omega})\right]}{Z_{2}}
$$

Não precisamos nos preocupar com a função de partição $Z_{2}$, já que ela é positiva. Se os demais termos forem positivos, toda a expressão é positiva. Lembrando da definição da energia $H_{2}$, podemos escrevê-la em termos de somas em $X_{i}$ e $Y_{i}$, como abaixo: 


$$
\begin{aligned}
H_{2}(\omega, \widetilde{\omega}) & =H_{\Lambda}(\omega)+H_{\Lambda}(\widetilde{\omega})=-\sum_{i \in \Lambda}\left\{\sum_{j \in \Lambda} \frac{1}{2} J_{i j} \omega_{i} \omega_{j}+h_{i} \omega_{i}+\sum_{j \in \Lambda} \frac{1}{2} J_{i j} \widetilde{\omega}_{i} \widetilde{\omega}_{j}+h_{i} \widetilde{\omega}_{i}\right\} \\
& =-\sum_{i \in \Lambda}\left\{\sum_{j \in \Lambda} \frac{1}{2} J_{i j}\left[\omega_{i} \omega_{j}+\widetilde{\omega}_{i} \widetilde{\omega}_{j}\right]+h_{i}\left[\omega_{i}+\widetilde{\omega}_{i}\right]\right\} \\
& =-\sum_{i \in \Lambda}\left\{\sum_{j \in \Lambda} J_{i j}\left[X_{i}(\omega, \widetilde{\omega}) X_{j}(\omega, \widetilde{\omega})+Y_{i}(\omega, \widetilde{\omega}) Y_{j}(\omega, \widetilde{\omega})\right]+h_{i} \sqrt{2} Y_{i}(\omega, \widetilde{\omega})\right\}
\end{aligned}
$$

Reescrevendo a identidade acima como uma igualdade de funções definidas em $\Omega_{\Lambda} \times \Omega_{\Lambda}$ obtemos uma expressão mais simples que é dada por

$$
H_{2}=-\sum_{i \in \Lambda}\left\{\sum_{j \in \Lambda} J_{i j}\left[X_{i} X_{j}+Y_{i} Y_{j}\right]+h_{i} \sqrt{2} Y_{i}\right\}
$$

O somatório acima pode ser substituído na expressão para $\left\langle X_{A} Y_{B}\right\rangle_{2}$ onde aparece $\exp \left[-\beta H_{2}(\omega, \widetilde{\omega})\right]$, cancelando ambos os sinais de menos. Expandindo essa exponencial em série de Taylor, $\left\langle X_{A} Y_{B}\right\rangle_{2}$ se decompõe em um somatório de termos da forma $X_{A} Y_{B}\left(H_{2}\right)^{n}$. Como o somatório inicial é tomado em $\omega, \widetilde{\omega} \in \Omega_{\Lambda}$, podemos alterar a ordem desse somatório, deixando em evidência os termos dependentes apenas de $\omega_{i}, \widetilde{\omega}_{i}$. O que resta é o produto de vários somatórios com a forma

$$
\sum_{\omega_{i}, \widetilde{\omega}_{i} \in\{-1,+1\}} X_{i}^{m}(\omega, \widetilde{\omega}) Y_{i}^{n}(\widetilde{\omega})
$$

sendo $m$ e $n$ dois números inteiros não-negativos. Assim, basta provar que para quaisquer inteiros $m, n \geq 0$ temos

$$
\sum_{\omega_{i}, \widetilde{\omega}_{i} \in\{-1,+1\}} X_{i}^{m}(\omega, \widetilde{\omega}) Y_{i}^{n}(\omega, \widetilde{\omega})=\sum_{\omega_{i}, \widetilde{\omega}_{i} \in\{-1,+1\}}\left(\frac{\omega_{i}-\widetilde{\omega}_{i}}{\sqrt{2}}\right)^{m}\left(\frac{\omega_{i}+\widetilde{\omega}_{i}}{\sqrt{2}}\right)^{n} \geq 0 .
$$

Se $m$ e $n$ forem pares, cada termo do somatório é não-negativo, logo a desigualdade é verdadeira nesse caso.

Já se $m$ for ímpar, os termos com $\omega_{i}=\widetilde{\omega}_{i}$ são nulos, restando apenas

$$
\left(\frac{+2}{\sqrt{2}}\right)^{m}\left(\frac{0}{\sqrt{2}}\right)^{n}+\left(\frac{-2}{\sqrt{2}}\right)^{m}\left(\frac{0}{\sqrt{2}}\right)^{n}=\left(\frac{2}{\sqrt{2}}\right)^{m}\left(\frac{0}{\sqrt{2}}\right)^{n}-\left(\frac{2}{\sqrt{2}}\right)^{m}\left(\frac{0}{\sqrt{2}}\right)^{n}=0 .
$$

Aqui temos um somatório nulo mesmo que $n=0$, logo a desigualdade 
é verdadeira também para $m$ ímpar. O caso $n$ ímpar é totalmente análogo ao $m$ ímpar. Sendo assim, a desigualdade original é verdadeira para todos os valores de $m$ e $n$, estando provada a desigualdade de GKS-2.

Em analogia ao apresentado após a demonstração da desigualdade de FKG, aqui apresentaremos uma consequência da desigualdade GKS-2 que terá uso no último capítulo. O corolário é assim enunciado:

Corolário 2.2.5. Num modelo de Ising ferromagnético a volume finito com campo externo $h_{i} \geq 0$ para todo sítio $i \in \Lambda$, a magnetização é uma função não decrescente das constantes de acoplamento $J_{i j}$.

Para demonstrar o corolário, calcularemos explicitamente $\frac{\partial}{\partial J_{i j}} m_{\Lambda}$, mostrando que como função de $J_{i j}$ esta derivada é não-negativa. Se o leitor acompanhou a demonstração do Corolário 2.2.3, esta demostração é totalmente análoga, com a única diferença de se utilizar no último passo a desigualdade de GKS-2 em lugar da de FKG.

Demonstração. A derivada de $m_{\Lambda}$ com respeito a $J_{i j}$ é dada por

$$
\frac{\partial}{\partial J_{i j}} m_{\Lambda}=\frac{\partial}{\partial J_{i j}} \int_{\Omega_{\Lambda}} \frac{\sum_{k \in \Lambda} \sigma_{k}}{|\Lambda|} \mathbb{P}_{\Lambda}(\sigma) d \kappa(\sigma)=|\Lambda|^{-1} \sum_{k \in \Lambda} \int_{\Omega_{\Lambda}} \sigma_{k} \frac{\partial}{\partial J_{i j}} \mathbb{P}_{\Lambda}(\sigma) d \kappa(\sigma) .
$$

Desta forma podemos reduzimos o problema a mostrar que, para cada $k \in \Lambda$, a integral $\int_{\Omega_{\Lambda}} \sigma_{k} \frac{\partial}{\partial J_{i j}} \mathbb{P}_{\Lambda}(\sigma) d \kappa(\sigma)$. é não-negativa. Como fizemos anteriormente, para cada $\sigma \in \Omega_{\Lambda}$ fixado, vamos calcular primeiro a derivada de $\mathbb{P}_{\Lambda}(\sigma)$ com respeito a $J_{i j}$.

$$
\begin{aligned}
\frac{\partial \mathbb{P}_{\Lambda}(\sigma)}{\partial J_{i j}}= & \frac{\partial}{\partial J_{i j}}\left[\frac{\exp \left(-\beta H_{\Lambda}(\sigma)\right)}{Z_{\Lambda}}\right]=\frac{Z_{\Lambda} \frac{\partial}{\partial J_{i j}} \exp \left(-\beta H_{\Lambda}(\sigma)\right)-\exp \left(-\beta H_{\Lambda}(\sigma)\right) \frac{\partial}{\partial J_{i j}} Z_{\Lambda}}{\left(Z_{\Lambda}\right)^{2}} \\
= & \frac{-\beta Z_{\Lambda} \exp \left(-\beta H_{\Lambda}(\sigma)\right) \frac{\partial}{\partial J_{i j}} H_{\Lambda}(\sigma)-\exp \left(-\beta H_{\Lambda}(\sigma)\right) \frac{\partial}{\partial J_{i j}} Z_{\Lambda}}{\left(Z_{\Lambda}\right)^{2}} \\
= & \frac{\beta Z_{\Lambda} \exp \left(-\beta H_{\Lambda}(\sigma)\right) \sigma_{i} \sigma_{j}-\beta \exp \left(-\beta H_{\Lambda}(\sigma)\right)\left\langle\sigma_{i} \sigma_{j}\right\rangle_{\Lambda}}{\left(Z_{\Lambda}\right)^{2}} \\
= & \frac{\beta \exp \left(-\beta H_{\Lambda}(\sigma)\right)}{Z_{\Lambda}}\left[\sigma_{i} \sigma_{j}-\left\langle\sigma_{i} \sigma_{j}\right\rangle_{\Lambda}\right] \\
= & \beta \mathbb{P}_{\Lambda}(\sigma)\left[\sigma_{i} \sigma_{j}-\left\langle\sigma_{i} \sigma_{j}\right\rangle_{\Lambda}\right] .
\end{aligned}
$$


Substituindo esta expressão na integral anterior ficamos com

$$
\beta \int_{\Omega_{\Lambda}} \sigma_{k}\left[\sigma_{i} \sigma_{j}-\left\langle\sigma_{i} \sigma_{j}\right\rangle_{\Lambda}\right] \mathbb{P}_{\Lambda}(\sigma) d \kappa(\sigma)=\beta\left(\left\langle\sigma_{i} \sigma_{j} \sigma_{k}\right\rangle_{\Lambda}-\left\langle\sigma_{i} \sigma_{j}\right\rangle_{\Lambda}\left\langle\sigma_{k}\right\rangle_{\Lambda}\right)
$$

Podemos usar a desigualdade de GKS-2 com $A=\{i, j\}$ e $B=\{k\}$ para concluir que a integral acima é não-negativa. E como observado anteriormente isto implica que $\partial m_{\Lambda} / \partial J_{i j} \geq 0$ e assim, está terminada a demonstração da desigualdade desejada.

A próxima desigualdade de correlação que vamos estabelecer e a Desigualdade GHS, provada pela primeira vez em 1970 por Griffiths, Hurst e Sherman em [14]. A prova apresentada aqui porém é baseada nos trabalhos [7] de Ellis e Monroe e [17] de Lebowitz. A técnica utilizada nestes trabalhos para provar a desigualdade é muito parecida com aquela que usamos para provar GKS-2, com a principal diferença sendo que ao invés de duplicarmos variáveis, desta vez vamos quadruplicá-las.

Fixado $\Lambda \subset \mathbb{Z}$ finito, vamos introduzir um sistema de spins quadruplicado com espaço de configuração $\Omega_{\Lambda}^{4} \equiv \Omega_{\Lambda} \times \Omega_{\Lambda} \times \Omega_{\Lambda} \times \Omega_{\Lambda}$. Vamos representar um ponto deste espaço por um quadrupla ordenada $(\omega, \sigma, \delta, \eta)$, onde cada uma das "coordenadas" $\omega, \sigma, \delta, \eta \in \Omega_{\Lambda}$. Definimos o Hamiltoniano $H_{\Lambda}^{(4)}: \Omega_{\Lambda}^{4} \rightarrow \mathbb{R}$ por

$$
H_{\Lambda}^{(4)}(\omega, \sigma, \delta, \eta)=H_{\Lambda}(\omega)+H_{\Lambda}(\sigma)+H_{\Lambda}(\delta)+H_{\Lambda}(\eta)
$$

onde $H_{\Lambda}$ é o Hamiltoniano do modelo de Ising dado por

$$
H_{\Lambda}(\sigma)=-\sum_{i \in \Lambda}\left\{\sum_{j \in \Lambda} \frac{1}{2} J_{i j} \sigma_{i} \sigma_{j}+h_{i} \sigma_{i}\right\} .
$$

Analogamente a notação introduzida na prova de GKS-2 vamos denotar por $\langle\cdot\rangle_{4}$ a esperança com relação à distribuição de probabilidades definida pelo Hamiltoniano $H_{\Lambda}^{(4)}$.

Lema 2.2.6. Para cada $i \in \Lambda$ defina as seguintes funções

$$
\begin{array}{ll}
A_{i}(\omega, \sigma, \delta, \eta)=\frac{\omega_{i}+\sigma_{i}+\delta_{i}+\eta_{i}}{2} & B_{i}(\omega, \sigma, \delta, \eta)=\frac{\omega_{i}+\sigma_{i}-\delta_{i}-\eta_{i}}{2} \\
C_{i}(\omega, \sigma, \delta, \eta)=\frac{\omega_{i}-\sigma_{i}+\delta_{i}-\eta_{i}}{2} & D_{i}(\omega, \sigma, \delta, \eta)=\frac{-\omega_{i}+\sigma_{i}+\delta_{i}-\eta_{i}}{2}
\end{array}
$$

Se no Hamiltoniano (2.14) cada $h_{i} \geq 0$, então para quaisquer subconjuntos 
não-vazios $\Delta, \Gamma, \Theta, \Upsilon \subset \Lambda$ temos que

$$
\left\langle A_{\Delta} B_{\Gamma} C_{\Theta} D_{\Upsilon}\right\rangle_{4} \geq 0
$$

onde a v.a. $A_{\Delta}=\prod_{i \in \Delta} A_{i}, \ldots, D_{\Upsilon}=\prod_{i \in \Upsilon} D_{i}$.

Demonstração. Para cada $i \in \Lambda$ fixado, podemos representar (2.15) matricialmente como segue

$$
\left(\begin{array}{c}
A_{i}(\omega, \sigma, \delta, \eta) \\
B_{i}(\omega, \sigma, \delta, \eta) \\
C_{i}(\omega, \sigma, \delta, \eta) \\
D_{i}(\omega, \sigma, \delta, \eta)
\end{array}\right)=\frac{1}{2}\left(\begin{array}{cccc}
1 & 1 & 1 & 1 \\
1 & 1 & -1 & -1 \\
1 & -1 & 1 & -1 \\
-1 & 1 & 1 & -1
\end{array}\right)\left(\begin{array}{c}
\omega_{i} \\
\sigma_{i} \\
\delta_{i} \\
\eta_{i}
\end{array}\right)
$$

É imediato verificar que as linhas da matriz acima são mutuamente ortogonais e portanto a matriz acima induz uma aplicação linear ortogonal em $\mathbb{R}^{4}$, como tais aplicações preservam produto interno podemos verificar que para quaisquer $i, j \in \Lambda$ que

$$
\omega_{i} \omega_{j}+\sigma_{i} \sigma_{j}+\delta_{i} \delta_{j}+\eta_{i} \eta_{j}=\frac{1}{2}\left(A_{i} A_{j}+B_{i} B_{j}+C_{i} C_{j}+D_{i} D_{j}\right),
$$

onde na igualdade acima deve ser entendido que as funções $A_{i}, \ldots, D_{i}$ estão calculadas no ponto $(\omega, \sigma, \delta, \eta)$. Portanto como igualdade de funções podemos escrever

$$
H_{\Lambda}^{(4)}=-\frac{1}{2} \sum_{i \in \Lambda}\left\{\sum_{j \in \Lambda} J_{i j}\left[A_{i} A_{j}+B_{i} B_{j}+C_{i} C_{j}+D_{i} D_{j}\right]+h_{i} A_{i}\right\} .
$$

A partir deste ponto seguimos as mesmas ideias empregadas na prova de GKS-2: expandimos a exponencial que aparece em $\left\langle A_{\Delta} B_{\Gamma} C_{\Theta} D_{\Upsilon}\right\rangle_{4}$ e usamos o teorema de Fubini. Feito isto é fácil ver que a não negatividade deste valor esperado segue se mostrarmos que a seguinte desigualdade é valida para quaisquer número inteiros positivos $k, l, m, n$.

$$
\int_{\Omega_{\Lambda}^{4}} A_{i}^{k}(x) B_{i}^{l}(x) C_{i}^{m}(x) D_{i}^{n}(x) d \kappa(x) \geq 0,
$$

onde $\kappa$ denota a medida de contagem em $\Omega_{\Lambda}^{4}$. Para provar que esta desigualdade é verdadeira observamos primeiro que por simetria a integral acima é zero a menos que $k, l, m, n$ tenham a mesma paridade. Quando a paridade é par o integrando é obviamente não negativo e a desigualdade 
certamente é válida. Quando a paridade é ímpar usamos uma propriedade especial dos spins de Ising que é $\omega_{i}^{2}=\sigma_{i}^{2}=\delta_{i}^{2}=\eta_{i}^{2}=1$, da seguinte maneira: fatoramos

$$
A_{i}^{k} B_{i}^{l} C_{i}^{m} D_{i}^{n}=A_{i}^{k-1} B_{i}^{l-1} C_{i}^{m-1} D_{i}^{n-1} \cdot A_{i} B_{i} C_{i} D_{i}
$$

e em seguida, notamos que para qualquer ponto $(\omega, \sigma, \delta, \eta)$ vale a seguinte igualdade

$$
\left(A_{i} \cdot B_{i} \cdot C_{i} \cdot D_{i}\right)(\omega, \sigma, \delta, \eta)=\frac{1}{4}\left(\omega_{i} \sigma_{i}-\delta_{i} \eta_{i}\right)^{2} .
$$

Desta forma temos que mesmo no caso de paridade ímpar a função no integrando $A_{i}^{k} B_{i}^{l} C_{i}^{m} D_{i}^{n}$ é pontualmente não-negativa e o lema esta provado.

Teorema 2.2.7 (Desigualdade GHS). Considere o modelo de Ising no volume $\Lambda$ com Hamiltoniano dado por

$$
H_{\Lambda}(\sigma)=-\sum_{i \in \Lambda}\left\{\sum_{j \in \Lambda} \frac{1}{2} J_{i j} \sigma_{i} \sigma_{j}+h_{i} \sigma_{i}\right\} .
$$

Seja $\langle\cdot\rangle_{\Lambda}$ o valor esperado com respeito a medida de Gibbs a volume $\Lambda$ determinada por $H_{\Lambda}$. Se $J_{i j}, h_{i} \geq 0$ para todo $i, j \in \Lambda$, então para quaisquer sítios $i, j, k \in \Lambda$ temos a seguinte desigualdade

$$
\left\langle\sigma_{i} \sigma_{j} \sigma_{k}\right\rangle_{\Lambda}-\left\langle\sigma_{i}\right\rangle_{\Lambda}\left\langle\sigma_{j} \sigma_{k}\right\rangle_{\Lambda}-\left\langle\sigma_{j}\right\rangle_{\Lambda}\left\langle\sigma_{i} \sigma_{k}\right\rangle_{\Lambda}-\left\langle\sigma_{k}\right\rangle_{\Lambda}\left\langle\sigma_{i} \sigma_{j}\right\rangle_{\Lambda}+2\left\langle\sigma_{i}\right\rangle_{\Lambda}\left\langle\sigma_{j}\right\rangle_{\Lambda}\left\langle\sigma_{k}\right\rangle_{\Lambda} \leq 0 .
$$

Demonstração. Defina as seguinte funções em $\Omega_{\Lambda}^{(4)}$

$$
\begin{aligned}
R_{i}(\omega, \sigma, \delta, \eta) & =\frac{\omega_{i}+\sigma_{i}}{\sqrt{2}} & S_{i}(\omega, \sigma, \delta, \eta) & =\frac{\omega_{i}-\sigma_{i}}{\sqrt{2}} \\
T_{i}(\omega, \sigma, \delta, \eta) & =\frac{\delta_{i}+\eta_{i}}{\sqrt{2}} & U_{i}(\omega, \sigma, \delta, \eta) & =\frac{\delta_{i}-\eta_{i}}{\sqrt{2}}
\end{aligned}
$$

Como observado por Lebowitz [17]

$$
\begin{gathered}
\left\langle\sigma_{i} \sigma_{j} \sigma_{k}\right\rangle_{\Lambda}-\left\langle\sigma_{i}\right\rangle_{\Lambda}\left\langle\sigma_{j} \sigma_{k}\right\rangle_{\Lambda}-\left\langle\sigma_{j}\right\rangle_{\Lambda}\left\langle\sigma_{i} \sigma_{k}\right\rangle_{\Lambda}-\left\langle\sigma_{k}\right\rangle_{\Lambda}\left\langle\sigma_{i} \sigma_{j}\right\rangle_{\Lambda}+2\left\langle\sigma_{i}\right\rangle_{\Lambda}\left\langle\sigma_{j}\right\rangle_{\Lambda}\left\langle\sigma_{k}\right\rangle_{\Lambda} \\
= \\
-\sqrt{2}\left\langle R_{k} U_{i} U_{j}-R_{k} S_{i} S_{j}\right\rangle_{\Lambda}^{(4)}
\end{gathered}
$$

onde $\langle\cdot\rangle_{\Lambda}^{(4)}$ é o valor esperado com respeito a medida de Gibbs determinada pelo Hamiltoniano quadruplicado (2.14). Para concluir a prova basta ob- 
servar que a última esperança da igualdade acima pode ser escrita em função das variáveis aleatórias $A_{i}, B_{i}, C_{i}, D_{i}$, definidas em (2.15), como segue

$$
\begin{gathered}
-\sqrt{2}\left\langle\frac{A_{k}+B_{k}}{\sqrt{2}}\left[\left(\frac{C_{i}+D_{i}}{\sqrt{2}}\right)\left(\frac{C_{j}+D_{j}}{\sqrt{2}}\right)-\left(\frac{C_{i}-D_{i}}{\sqrt{2}}\right)\left(\frac{C_{j}-D_{j}}{\sqrt{2}}\right)\right]\right\rangle_{\Lambda}^{(4)} \\
= \\
-\left\langle\left(A_{k}+B_{k}\right)\left(C_{i} D_{j}+C_{j} D_{i}\right)\right\rangle_{\Lambda}^{(4)} .
\end{gathered}
$$

Já que este último valor esperado é não negativo, pelo Lema 2.2.6, a desigualdade GHS está demonstrada.

A principal aplicação da desigualdade GHS é mostrar que a magnetização específica a volume finito é uma função côncava, quando vista como função apenas do parâmetro $h$ (campo externo). Este cálculo é feito com detalhes ao longo da prova do Teorema 3.4.3.

Além desta aplicação no modelos de Ising e Curie-Weiss é importante mencionar que a desigualdade GHS pode ser também aplicada para determinar propriedades de concavidade de certas equações diferenciais a derivadas parciais e certos processos de difusão. Condições necessárias e suficientes para a validade desta desigualdade são apresentadas no importante trabalho [8] de Ellis e Newman de 1978, bem como algumas aplicações desta desigualdade em EDP's e processos de difusão.

\subsection{Condições Exteriores}

Na Seção 1.3 apresentamos o modelo a volume finito e a magnetização nesse modelo. O modelo ali definido é denominado com mais precisão por modelo de Ising a volume finito com condições de fronteira(ou exteriores) livres. O volume $\Lambda$ era considerado ali como um subconjunto de $\mathbb{Z}$. Poderíamos, então, fixar os spins exteriores a $\Lambda$ e definir a interação entre esses spins fixados e os spins de $\Lambda$. Esse outro modelo, com condições exteriores definidas, é uma espécie de generalização do anterior. Como no modelo inicialmente exposto não havia interação dos spins de $\Lambda$ com entes exteriores, diz-se que ele tem "condições exteriores livres". Assim, redefiniremos o modelo de forma a acrescentar as citadas condições exteriores.

Precisaremos acrescentar no hamiltoniano os termos de interação com os spins exteriores. Os sítios exteriores a $\Lambda$ são os elementos de $\Lambda^{c} \equiv \mathbb{Z} \backslash \Lambda$. 
Logo, o estado fixado dos spins exteriores será um elemento do conjunto $\Omega_{\Lambda^{c}}=\{-1,+1\}^{\Lambda^{c}}$. Esse estado exterior será denominado $\widetilde{\sigma}$. Podemos agora escrever (formalmente pois, não lidamos ainda com as questões de convergência) o novo hamiltoniano de um modelo de Ising finito com condições exteriores $\widetilde{\sigma}$ como segue

$$
H_{\Lambda}^{\widetilde{\sigma}}(\sigma)=\sum_{i \in \Lambda}\left\{\sum_{j \in \Lambda}-\frac{1}{2} J_{i j} \sigma_{i} \sigma_{j}-h_{i} \sigma_{i}-\sum_{j \in \mathbb{Z} \backslash \Lambda} J_{i j} \widetilde{\sigma}_{j} \sigma_{i}\right\}
$$

O $\widetilde{\sigma}$ superescrito em $H_{\Lambda}^{\widetilde{\sigma}}$ será usado para nos lembrar que é um hamiltoniano de um modelo com condição exterior $\widetilde{\sigma}$. É natural que os coeficientes $J_{i j}$ tenham sido estendidos para $i, j \in \mathbb{Z}$ com $J_{i i}=0, \forall i \in \Lambda$. Podemos perceber que a interação com os spins externos a $\Lambda$ age de forma muito similar a um campo externo, pois um sistema com condições externas livres e campo externo

$$
\widetilde{h}_{i}=h_{i}+\sum_{j \in \mathbb{Z} \backslash \Lambda} J_{i j} \widetilde{\sigma}_{j}
$$

tem exatamente o mesmo hamiltoniano. Logo, tanto faz definir um sistema com condição exterior $\widetilde{\sigma}$ e campo externo $h_{i}$ ou um sistema com condição exterior livre e campo externo $\widetilde{h}_{i}$.

Quanto aos estados exteriores, aqueles de maior interesse têm todos seus spins $\sigma_{i}$ alinhados, ou seja, todos os spins valendo +1 ou -1 . Esses estados exteriores serão denotados estado + , quando para todo $i \in \Lambda^{c}$, $\sigma_{i}=+1$ e estado - no caso simétrico.

Na seção seguinte nos restringiremos a mostrar a existência dos limites da magnetização quando $\Lambda \rightarrow \mathbb{Z}$ para essas duas condições exteriores especiais $+\mathrm{e}-$. Como as duas condições são totalmente simétricas, trabalharemos apenas com + .

\subsection{Magnetização a Volume Finito}

Usando o corolário da desigualdade de FKG (Corolário 2.2.3) podemos demonstrar a convergência da magnetização quando $\Lambda \rightarrow \mathbb{Z}$ para os casos com interação dependente apenas da distância entre os spins, $J_{i j}=J(\mid i-$ $j \mid$ ), campo externo constante $h_{i}=h$ e condições exteriores + ou -. Como a demonstração para o caso - é totalmente análoga, exporemos aqui apenas o caso + . 
O artifício a ser utilizado é comum a várias outras questões sobre modelos de Ising. Trata-se de fazer o campo externo em alguns sítios escolhidos tender a $+\infty$. Intuitivamente, isso obriga os spins naqueles sítios a se "alinharem" com o campo externo, sendo positivos quase certamente. O sistema alterado, então, se comporta como um sistema com spins positivos fixos nos sítios onde o campo foi aumentado. Assim, é possível utilizar o Corolário 2.2.3 para mostrar a não-diminuição do valor esperado de uma função crescente qualquer quando fixamos um dos spins da rede no sentido +1 , já que fixar um spin $i$ seria equivalente a fazer $h_{i} \rightarrow \infty$. Vamos agora tornar essa ideia intuitiva algo mais precisa.

Deste ponto em diante, vamos assumir que $J_{i j}$ satisfaz

$$
\sup _{i \in \mathbb{Z}} \sum_{j \in \mathbb{Z}}\left|J_{i j}\right|<\infty .
$$

Essa hipótese é conveniente para que possamos argumentar que todas as somas envolvendo $J_{i j}$ que aparecem no restante desta seção sejam absolutamente convergentes.

Observando como a alteração do campo externo $h_{\alpha}$ influencia na energia de um estado $\sigma$ podemos compreender melhor o que acontece quando $h_{\alpha} \rightarrow \infty$. A energia do estado $\sigma$ com condições de contorno + é dada por

$$
H_{\Lambda}^{+}(\sigma)=\sum_{i \in \Lambda}\left\{\sum_{j \in \Lambda}-\frac{1}{2} J_{i j} \sigma_{i} \sigma_{j}-h_{i} \sigma_{i}-\sum_{j \in \mathbb{Z} \backslash \Lambda} J_{i j} \sigma_{i}\right\}
$$

Elegemos-se, então, um sítio $\alpha$ fixado. Quando fazemos $h_{\alpha} \rightarrow \infty$, o termo $h_{\alpha} \sigma_{\alpha}$ do somatório acima faz a energia do estado $\sigma$ tender a $-\infty$ se $\sigma_{\alpha}=+1$. Por outro lado, se $\sigma$ é tal que $\sigma_{\alpha}=-1$ então a energia do estado $\sigma$ quando $h_{\alpha} \rightarrow+\infty$ tende a $+\infty$. Já que a probabilidade do estado $\sigma$ é dada por

$$
\mathbb{P}_{\Lambda}^{+}(\sigma)=\frac{e^{-H_{\Lambda}^{+}(\sigma)}}{Z_{\Lambda}^{+}}=\frac{e^{-H_{\Lambda}^{+}(\sigma)}}{\sum_{\omega \in \Omega_{\Lambda}} e^{-H_{\Lambda}^{+}(\omega)}}
$$

Evidentemente, se $\sigma_{\alpha}=-1$, o numerador da probabilidade do estado $\sigma$ tende a zero e como $\lim _{h_{\alpha} \rightarrow+\infty} Z_{\Lambda}^{+}=+\infty$ temos que $\mathbb{P}_{\Lambda}^{+}(\sigma) \rightarrow 0$. Por outro lado, se $\sigma_{\alpha}=+1$, no numerador aparece um fator multiplicativo $\exp \left(h_{\alpha}\right)$ que fará $\exp \left(-H_{\Lambda}^{+}(\sigma)\right) \rightarrow+\infty$. Contudo, o somatório no denominador tem todos os termos com $\omega_{\alpha}=-1$ tendendo a zero e todos os termos com $\omega_{\alpha}=+1$ com o mesmo fator multiplicativo $\exp \left(h_{\alpha}\right)$ que pode ser cancelado com o equivalente do numerador. 
Desse modo, se denotamos por $\Lambda^{\prime}=\Lambda \backslash\{\alpha\}$ a caixa sem o sítio $\alpha, \sigma^{\prime}$ a restrição de $\sigma$ à caixa $\Lambda^{\prime}, H_{\Lambda^{\prime}}^{+}$e $P_{\Lambda^{\prime}}^{+}$respectivamente a energia e a probabilidade para um sistema definido em $\Lambda^{\prime}$ com condições exteriores + e spin $\alpha$ fixo em +1 , chegamos à seguinte conclusão

$$
\mathbb{P}_{\Lambda}^{+}(\sigma)=\frac{e^{-H_{\Lambda}^{+}(\sigma)}}{Z_{\Lambda}^{+}} \underset{h_{\alpha} \rightarrow+\infty}{\longrightarrow} \begin{cases}\frac{\exp \left[-H_{\Lambda^{\prime}}^{+}\left(\sigma^{\prime}\right)\right]}{Z_{\Lambda^{\prime}}}=\mathbb{P}_{\Lambda^{\prime}}^{+}\left(\sigma^{\prime}\right), & \text { se } \sigma_{\alpha}=+1 \\ 0, & \text { se } \sigma_{\alpha}=-1\end{cases}
$$

Pode-se concluir com a expressão acima que quando $h_{\alpha} \rightarrow \infty$ o sistema definido em $\Lambda$ com hamiltoniano $H_{\Lambda}^{+}$é totalmente análogo a um sistema definido em $\Lambda^{\prime}$ e com Hamiltoniano $H_{\Lambda^{\prime}}^{+}$onde $\sigma_{\alpha}=+1$ é fixado. De fato, pelo Teorema da Convergência Dominada para toda função integrável $f$ : $\Omega_{\Lambda} \rightarrow \mathbb{R}$ com respeito a $\mathbb{P}_{\Lambda}$ temos que

$$
\begin{aligned}
\lim _{h_{\alpha} \rightarrow+\infty}\langle f\rangle_{\Lambda}^{+} & =\lim _{h_{\alpha} \rightarrow+\infty} \int_{\Omega_{\Lambda}} f(\sigma) P_{\Lambda}(\sigma) d \kappa(\sigma) \\
& =\int_{\Omega_{\Lambda}} 1_{\left\{\sigma_{\alpha}=+1\right\}} f(\sigma) P_{\Lambda^{\prime}}\left(\sigma^{\prime}\right) d \kappa(\sigma) \\
& =\langle f(\cdot,+1)\rangle_{\Lambda^{\prime}}^{+} .
\end{aligned}
$$

Em particular se $f: \Omega_{\Lambda} \rightarrow \mathbb{R}$ não depende da variável $\sigma_{\alpha}$ então podemos escrever (com um pequeno abuso de notação)

$$
\lim _{h_{\alpha} \rightarrow+\infty}\langle f\rangle_{\Lambda}^{+}=\langle f\rangle_{\Lambda^{\prime}}^{+}
$$

Com esse resultado, continuamos com a análise relativa ao limite da magnetização com $\Lambda \rightarrow \mathbb{Z}$

A sequência cuja convergência gostaríamos e analisar é a sequência das magnetizações específicas de sistemas finitos com condições exteriores + e caixas de tamanhos crescentes. O natural seria utilizar a sequência de caixas da forma $\mathbb{Z} \cap[-N,+N]$, contudo, para facilitar a exposição, como a magnetização específica não se altera ao transladarmos por um número finito de sítios o sistema como um todo, utilizaremos a sequência de caixas $\Lambda_{N}=\mathbb{Z} \cap[1,+N]$. Para não carregar a notação vamos denotar a magnetização específica do o sistema definido em $\Lambda_{N}$ e com condições exteriores positiva, isto é, $+\left(\operatorname{com} \beta, h\right.$ e $J_{i j} \equiv J(|i-j|)$ fixos) por

$$
m_{N}=\frac{1}{N}\left\langle S_{N}\right\rangle_{\Lambda_{N}}^{+}=\left\langle\frac{1}{N} \sum_{i \in \Lambda_{N}} \sigma_{i}\right\rangle_{\Lambda_{N}}^{+} .
$$


O objetivo desta seção é mostrar a convergência da sequência de números reais $m_{N}$. Para tanto precisaremos do Lema de Fekete, que garante a convergência da média de sequências subaditivas, como posto abaixo.

Definição 2.4.1 (Sequência subaditiva). Uma sequência de números reais $a_{N}, N \in \mathbb{N}^{*}$ é dita subaditiva se, para todo $M, N \in \mathbb{N}^{*}, a_{M+N} \leq a_{M}+a_{N}$.

Lema 2.4.1 (Lema de Fekete). Dada uma sequência subaditiva $\left\{a_{N}\right\}_{N}, a$ sequência $\left\{a_{N} / N\right\}_{N}$ converge e

$$
\lim _{N \rightarrow \infty} \frac{a_{N}}{N}=\inf \frac{a_{N}}{N}
$$

Uma vez provada a subaditividade da sequência $\left\{\left\langle S_{N}\right\rangle_{\Lambda_{N}}\right\}_{N}$, seria possível aplicar o Lema de Fekete, chegando a conclusão da convergência da magnetização no limite infinito $m=\lim _{N \rightarrow \infty} m_{N}$. Para provar a citada subaditividade, tomemos o modelo definido na caixa $\Lambda_{M+N}$ com condições exteriores + . Dividiremos esse sistema em duas caixas menores, a primeira, a ser identificada por $\Delta_{M}$, conterá os spins de índices 1 até $M$. A outra, contendo os $N$ demais spins, de $M+1$ até $M+N$, será $\Delta_{N}$. Equivalentemente, a soma $\left\langle S_{M+N}\right\rangle_{\Lambda_{M+N}}$ será dividida em duas partes, já que

$$
\left\langle S_{M+N}\right\rangle_{\Lambda_{M+N}}^{+}=\left\langle\sum_{i \in \Lambda_{M+N}} \sigma_{i}\right\rangle_{\Lambda_{M+N}}^{+}=\left\langle\sum_{i \in \Delta_{M}} \sigma_{i}\right\rangle_{\Lambda_{M+N}}^{+}+\left\langle\sum_{i \in \Delta_{N}} \sigma_{i}\right\rangle_{\Lambda_{M+N}}^{+} .
$$

Em seguida, se alteramos o sistema de modo a introduzir campo infinito nos sítios de $\Delta_{N}$, ou seja, manter constantes todos os demais parâmetros, mas alterar $\left\{h_{i}\right\}$ fazendo $h_{i} \rightarrow+\infty$ para os sítios $i \in \Delta_{N}$, temos um sistema resultante com spins em $\Delta_{N}$ fixos em +1 . A restrição a $\Delta_{M}$ do sistema resultante tem spins com interação $J(i-j)$ dentro de $\Delta_{M}$, e que também interagem com spins fixos em +1 fora de $\Delta_{M}$. Portanto, o sistema resultante é exatamente igual ao sistema definido em $\Lambda_{M}=\Delta_{M}$ e com condições exteriores + . Como apenas aumentou-se o campo externo $h_{i}$ em alguns sítios, o Corolário 2.2.3 da Desigualdade de FKG nos garante que não há diminuição da função crescente $\left\langle\sum_{i \in \Delta_{M}} \sigma_{i}\right\rangle_{\Lambda_{M+N}}^{+}$. Em outras palavras,

$$
\left\langle\sum_{i \in \Delta_{M}} \sigma_{i}\right\rangle_{\Lambda_{M+N}}^{+} \leq\left\langle\sum_{i \in \Lambda_{M}} \sigma_{i}\right\rangle_{\Lambda_{M}}^{+}=\left\langle S_{M}\right\rangle_{\Lambda_{M}}^{+}
$$

Vamos analisar agora a segunda parcela no termo à extrema direita de (2.17). A diferença da análise deste termo para a do caso anterior é que 
$\Delta_{N}=\mathbb{Z} \cap[M+1, M+N]$, que a rigor é diferente $\Lambda_{N}=\mathbb{Z} \cap[1, N]$. Isso não gera problema para comparação, pois estamos supondo que nossa interação $J_{i j} \equiv J(|i-j|)$, isto é depende apenas da distância entre entre pares de spins e o campo externo $h$ é constante. Sendo assim, é possível transladar todo o sistema de $\Delta_{N}=\mathbb{Z} \cap[M+1, M+N]$ para $\Lambda_{N}=\mathbb{Z} \cap[1, N]$ sem alterar a soma dos spins na caixa sob análise. E, repetindo o argumento do caso anterior obtemos a seguinte desigualdade

$$
\left\langle\sum_{i \in \Delta_{N}} \sigma_{i}\right\rangle_{\Lambda_{M+N}}^{+} \leq\left\langle\sum_{i \in \Lambda_{N}} \sigma_{i}\right\rangle_{\Lambda_{N}}^{+}=\left\langle S_{N}\right\rangle_{\Lambda_{N}}^{+} .
$$

Juntando a última expressão com as duas desigualdades anteriores, podemos concluir que

$$
\left\langle S_{M+N}\right\rangle_{\Lambda_{M+N}}^{+} \leq\left\langle S_{M}\right\rangle_{\Lambda_{M}}^{+}+\left\langle S_{N}\right\rangle_{\Lambda_{N}}^{+}
$$

Assim a sequência de números reais $\left\{\left\langle S_{N}\right\rangle_{\Lambda_{N}}^{+}\right\}_{N}$ é, pois, subaditiva. Pelo Lema de Fekete, com $a_{N}=\left\langle S_{N}\right\rangle_{\Lambda_{N}}^{+}$temos que existe o limite da magnetização específica

$$
m=\lim _{N \rightarrow \infty} m_{N}=\lim _{N \rightarrow \infty} \frac{1}{N}\left\langle S_{N}\right\rangle_{\Lambda_{N}}^{+}=\inf _{N \in \mathbb{N}^{*}} \frac{1}{N}\left\langle S_{N}\right\rangle_{\Lambda_{N}}^{+} .
$$

Esse resultado será enunciado mais a frente como um lema para posterior referência.

Utilizando o mesmo raciocínio, é possível também provar a existência do limite da magnetização específica para o caso de condições de contorno livre, notação $m_{N, h}^{f}$, onde $m_{N, h}^{f}=\left\langle S_{N} / N\right\rangle_{\Lambda_{N}, h}^{f}$ com o superescrito $f$ denotando a condição de contorno livre ou "free". Vamos provar a existência do limite neste caso com campo externo $h>0$. Para isto será usado o corolário da Desigualdade GKS-2 (Corolário 2.2.5). Com ele vamos mostrar que a sequência $\left\{\left\langle S_{N}\right\rangle_{\Lambda_{N}, 0}\right\}_{N}$ é superaditiva, isto é,

$$
\left\langle S_{M+N}\right\rangle_{\Lambda_{M+N}, h}^{f} \geq\left\langle S_{M}\right\rangle_{\Lambda_{M}, h}^{f}+\left\langle S_{N}\right\rangle_{\Lambda_{N}, h}^{f}
$$

Para tal, novamente separaremos a caixa $\Lambda_{M+N}$ em suas duas partes $\Delta_{M}$ e $\Delta_{N}$. Em seguida, constrói-se um novo sistema com $J_{i j}=0$ sempre que $i \in \Delta_{M}$ e $j \in \Delta_{N}$. Desta forma anulamos a interação dos spins entre dos sítios $\Delta_{M}$ e $\Delta_{N}$ e portanto o que obtemos são dois sistemas independentes. Assim, após se "desligadas" estas interações, as duas partes restantes 
são totalmente equivalentes (a menos de uma translação) a dois sistemas independentes definidos em $\Lambda_{M}$ e $\Lambda_{N}$.

Como houve apenas a redução de algumas interações $J_{i j}$, o corolário da desigualdade de GKS-2 nos garante que não há aumento na magnetização em nenhum dos subconjuntos $\Delta_{M}$ e $\Delta_{N}$. Conclui-se, como desejado, que $\left\langle S_{M+N}\right\rangle_{\Lambda_{M+N}, h}^{f} \geq\left\langle S_{M}\right\rangle_{\Lambda_{M}, h}^{f}+\left\langle S_{N}\right\rangle_{\Lambda_{N}, h}^{f}$. Usando agora o Lema de Fekete para sequências superaditivas podemos garantir a convergência da sequência $\left\{\left\langle S_{N}\right\rangle_{\Lambda_{N}, h}^{f} / N\right\}_{N}$.

Lema 2.4.2. Para um modelo de Ising finito definido em uma caixa simétrica $\Lambda_{N}=\mathbb{Z} \cap[-N,+N]$, com constantes de acoplamento invariantes por translação $J_{i j}=J(|i-j|)$, campo magnético constante $h_{i}=h$ e condições exteriores livres, positiva(+) e negativa(-), fixado qualquer valor do inverso da temperatura $\beta$ a sequência das magnetizações específicas $m_{N}=\left\langle S_{N} / N\right\rangle_{\Lambda_{N}}$ converge para $m=\lim _{N \rightarrow \infty} m_{N}$.

\subsection{Algumas Propriedades de $S_{N} / N$}

Seguindo o raciocínio da seção anterior, tomamos uma sequência de modelos de Ising a volume finito com condições exteriores livres definidos em $\Lambda_{N}=\mathbb{Z} \cap[0, N]$. Principal diferença dos modelos considerados nesta e na seção anterior, é que aqui vamos assumir que $h_{i}=h>0$, ou seja, campo externo constante e positivo. Já mostramos na seção anterior a existência da magnetização $m$ no limite $N \rightarrow \infty$. Nesta seção vamos estudar o comportamento de $\mathbb{P}_{\Lambda_{N}, h}$ no limite termodinâmico $(N \rightarrow \infty)$.

Com os resultados até aqui apresentados, é possível verificar que

$$
m_{\beta, 0}=\lim _{N \rightarrow \infty} m_{\Lambda_{N}, \beta, 0}=\lim _{N \rightarrow \infty} 0=0,
$$

pois vimos na Seção 1.3 que se $h=0$ então $m_{\Lambda_{N}, \beta, 0}=0$,

Como a desigualdade de FKG nos assegura que o aumento no campo magnético não diminui a magnetização, temos que se $h \geq 0$ então $m_{\Lambda_{N}, \beta, h} \geq$ 0 para todo $N$ e portanto o limite também é não-negativo, isto é, $m_{\beta, h} \geq$ 0 . Lembrando que $m_{\beta, h}=\lim _{N \rightarrow \infty}\left\langle S_{N} / N\right\rangle_{\Lambda_{N}, h}$, podemos afirmar que o valor esperado da sequência de variáveis aleatórias $S_{N} / N$ converge um valor não negativo.

Grosseiramente falando, a principal meta desta seção é mostrar que além de ter média convergindo para um valor não-negativo, no "Limite 
Termodinâmico" a variável aleatória $S_{N} / N$ nunca se alinha contra o campo externo $h$. De maneira mais precisa, vamos mostrar que se $h>0$ então

$$
\lim _{N \rightarrow \infty} \mathbb{P}_{\Lambda_{N}, \beta, h}\left(\frac{S_{N}}{N}<-a\right)=0
$$

para qualquer valor positivo $a \in(0,1]$.

Para facilitar a notação da prova do fato mencionado acima, vamos omitir a dependência da medida de Gibbs com respeito ao parâmetro $\beta$ e escrever simplesmente, para todo evento $A$,

$$
\mathbb{P}_{\Lambda_{N}, \beta, h}(A) \equiv \mathbb{P}_{\Lambda_{N}, h}(A)
$$

Para demonstrar o resultado enunciado acima vamos começar obtendo cotas superiores para

$$
\mathbb{P}_{\Lambda_{N}, h}\left(\frac{S_{N}}{N} \leq-a\right)=\mathbb{P}_{\Lambda_{N}, h}\left(S_{N} \leq-a N\right) .
$$

Dado um estado $\sigma$ pertencente ao evento $E_{N, a}=\left\{\sigma \in \Omega_{N} \mid S_{N} \leq-a N\right\}$, existe um estado simétrico $-\sigma$, elemento do evento $-E_{N, a}=\left\{\sigma \in \Omega_{N} \mid S_{N} \geq\right.$ $+a N\}$. Podemos calcular a diferença entre as energias dos estados $\sigma \mathrm{e}-\sigma$ como mostrado abaixo:

$$
\begin{aligned}
H_{\Lambda}(\sigma)-H_{\Lambda}(-\sigma)= & -\sum_{i, j \in \Lambda}-\frac{1}{2} J_{i j} \sigma_{i} \sigma_{j}-h \sum_{i \in \Lambda} \sigma_{i} \\
& +\sum_{i, j \in \Lambda} \frac{1}{2} J_{i j}\left(-\sigma_{i}\right)\left(-\sigma_{j}\right)+h \sum_{i \in \Lambda}\left(-\sigma_{i}\right) \\
= & -2 h \sum_{i \in \Lambda} \sigma_{i} \\
= & -2 h S_{N}(\sigma) .
\end{aligned}
$$

Observe que ao passar de $\sigma$ para $-\sigma$ todos os spins são invertidos, a energia de interação entre qualquer par $(i, j)$, representada pelo termo $-\frac{1}{2} J_{i j} \sigma_{i} \sigma_{j}$, fica inalterada, pois $\sigma_{i} \sigma_{j}=\left(-\sigma_{i}\right)\left(-\sigma_{j}\right)$. Por outro lado, a energia de interação com o campo externo $h$, que é $h S_{N}(\sigma)$ para o estado $\sigma$, passa a ser $-h S_{N}(\sigma)$ para o estado $-\sigma$.

Portanto para todo $\sigma \in E_{N, a}$, temos que

$$
H_{\Lambda}(\sigma)-H_{\Lambda}(-\sigma) \geq+2 h a N .
$$


Assim, o estado $\sigma$, por ter a maioria dos spins alinhados contra o campo externo, tem energia pelo menos $2 h a N>0$ maior que o estado $-\sigma$. Como os estados com menor energia são mais prováveis, a comparação das probabilidades desses dois estados simétricos nos dá

$$
\frac{\mathbb{P}_{\Lambda_{N}, h}(\sigma)}{\mathbb{P}_{\Lambda_{N}, h}(-\sigma)}=\frac{\exp \left[-\beta H_{\Lambda_{N}, h}(\sigma)\right]}{\exp \left[-\beta H_{\Lambda_{N}, h}(-\sigma)\right]}=\exp \left(2 \beta h S_{N}(\sigma)\right) \leq \exp (-2 \beta h a N) .
$$

Mas a desigualdade acima vale para todo estado $\sigma \in E_{N, a}$, logo vale também quando comparamos os eventos $E_{N, a}$ e $-E_{N, a}$. Já que a probabilidade de qualquer evento está limitada por 1 , temos que $\mathbb{P}_{\Lambda_{N}, \beta, h}\left(-E_{N, a}\right) \leq 1$, logo podemos concluir que

$$
\mathbb{P}_{\Lambda_{N}, h}\left(E_{N, a}\right) \leq \exp (-2 \beta h a N) \cdot \mathbb{P}_{\Lambda_{N}, h}\left(-E_{N, a}\right) \leq \exp (-2 \beta h a N) .
$$

Tomando o limsup em ambos os lado da desigualdade acima, ficamos com

$$
\limsup _{N \rightarrow \infty} \mathbb{P}_{\Lambda_{N}, h}\left(\frac{S_{N}}{N} \leq-a\right) \leq \lim _{N \rightarrow \infty} \exp (-2 \beta h a N)=0 .
$$

Já que a medida de qualquer conjunto é não negativa e o limite superior é zero, podemos afirmar que o seguinte limite existe

$$
\lim _{N \rightarrow \infty} \mathbb{P}_{\Lambda_{N}, h}\left(\frac{S_{N}}{N} \leq-a\right)=0 .
$$

Esse resultado nos será útil no último capítulo, pois lá mostraremos que, para um certo sistema que atende às hipóteses tanto desta seção como da anterior (2.4),

$$
\liminf _{N \rightarrow \infty}\left\langle\left(\frac{S_{N}}{N}\right)^{2}\right\rangle_{\Lambda_{N}, h}=m^{(2)}>0 .
$$

Uma vez obtida a desigualdade acima, é possível aplicar o resultado provado nesta seção para mostrar que

$$
\liminf _{N \rightarrow \infty}\left\langle\left(\frac{S_{N}}{N}\right)^{2}\right\rangle_{\Lambda_{N}, h}=m^{(2)} \Longrightarrow m(\beta, h)=\lim _{N \rightarrow \infty}\left\langle\frac{S_{N}}{N}\right\rangle_{\Lambda_{N}, h} \geq m^{(2)} .
$$

Para mostrar a implicação acima, vamos usar a definição do valor esperado $\left\langle\left(S_{N} / N\right)^{2}\right\rangle_{\Lambda_{N}, h}$ e uma decomposição apropriada do espaço $\Omega_{\Lambda}$ em três subconjuntos, que serão definidos de acordo com o valor de $S_{N} / N$ como 
segue

$$
\begin{aligned}
\left\langle\left(\frac{S_{N}}{N}\right)^{2}\right\rangle_{\Lambda_{N}, h}= & \int_{\Omega_{\Lambda_{N}}}\left(\frac{S_{N}(\sigma)}{N}\right)^{2} \mathbb{P}_{\Lambda_{N}, \beta, h}(\sigma) d \kappa(\sigma) \\
= & \int_{\frac{S_{N}}{N}<-a}\left(\frac{S_{N}(\sigma)}{N}\right)^{2} \mathbb{P}_{\Lambda_{N}, \beta, h}(\sigma) d \kappa(\sigma)+\int_{\left|\frac{S_{N} \mid \leq a}{N}\right|}\left(\frac{S_{N}(\sigma)}{N}\right)^{2} \mathbb{P}_{\Lambda_{N}, \beta, h}(\sigma) d \kappa(\sigma) \\
& +\int_{S_{N} / N>+a}\left(\frac{S_{N}(\sigma)}{N}\right)^{2} \mathbb{P}_{\Lambda_{N}, \beta, h}(\sigma) d \kappa(\sigma) .
\end{aligned}
$$

Observe que o limite inferior da integral sobre $\left\{S_{N} / N<-a\right\}$ é zero. De fato, basta observar que $\left|S_{N} / N\right| \leq 1$ e usar a igualdade (2.21)

$$
\begin{aligned}
\liminf _{N \rightarrow \infty} \int_{\frac{S_{N}}{N}<-a}\left(\frac{S_{N}(\sigma)}{N}\right)^{2} \mathbb{P}_{\Lambda_{N}, \beta, h}(\sigma) d \kappa(\sigma) & \leq \liminf _{N \rightarrow \infty} \int_{\frac{S_{N}<-a}{N}} \mathbb{P}_{\Lambda_{N}, \beta, h}(\sigma) d \kappa(\sigma) \\
& =\liminf _{N \rightarrow \infty} \mathbb{P}_{\Lambda_{N}, \beta, h}\left(\frac{S_{N}}{N}<-a\right) \\
& =0 .
\end{aligned}
$$

Para a integral sobre $\left\{\left|S_{N} / N\right| \leq a\right\}$ usamos que a medida $\mathbb{P}_{\Lambda_{N}, h}$ é limitada por 1 e que o integrando é limitado por $a^{2}$, assim,

$$
\int_{\left|\frac{S_{N}}{N}\right| \leq a}\left(\frac{S_{N}(\sigma)}{N}\right)^{2} \mathbb{P}_{\Lambda_{N}, \beta, h}(\sigma) d \kappa(\sigma) \leq a^{2}
$$

Como estamos assumindo

$$
m^{(2)}=\liminf _{N \rightarrow \infty}\left\langle\left(\frac{S_{N}}{N}\right)^{2}\right\rangle_{\Lambda_{N}, h},
$$

temos que o limite inferior sobre toda a expressão de $\left\langle\left(S_{N} / N\right)^{2}\right\rangle_{\Lambda_{N}, h}$ é cotado superiormente por

$$
\begin{aligned}
m^{(2)}=\liminf _{N \rightarrow \infty}\left\langle\left(\frac{S_{N}}{N}\right)^{2}\right\rangle_{\Lambda_{N}, h}= & \liminf _{N \rightarrow \infty} \int_{\left|\frac{S_{N}}{N}\right| \leq a}\left(\frac{S_{N}(\sigma)}{N}\right)^{2} \mathbb{P}_{\Lambda_{N}, \beta, h}(\sigma) d \sigma \\
& +\liminf _{N \rightarrow \infty} \int_{\frac{S_{N}}{N}>+a}\left(\frac{S_{N}(\sigma)}{N}\right)^{2} \mathbb{P}_{\Lambda_{N}, \beta, h}(\sigma) d \sigma \\
\leq & a^{2}+\liminf _{N \rightarrow \infty} \int_{\frac{S_{N}}{N}>+a} \frac{S_{N}(\sigma)}{N} \mathbb{P}_{\Lambda_{N}, \beta, h}(\sigma) d \sigma
\end{aligned}
$$


onde na última desigualdade usamos $0 \leq S_{N} / N \leq 1$, nos permitindo concluir, neste caso, que $S_{N}^{2} / N^{2} \leq S_{N} / N$.

Da desigualdade acima segue imediatamente

$$
\liminf _{N \rightarrow \infty} \int_{\frac{S_{N}}{N}>+a} \frac{S_{N}(\sigma)}{N} \mathbb{P}_{\Lambda_{N}, \beta, h}(\sigma) d \sigma \geq m^{(2)}-a^{2} .
$$

Aplicando exatamente o mesmo raciocínio a $\liminf _{N \rightarrow \infty}\left\langle S_{N} / N\right\rangle_{\Lambda_{N}, h}$ e a desigualdade acima, concluímos que

$$
\begin{aligned}
\lim _{N \rightarrow \infty}\left\langle\frac{S_{N}}{N}\right\rangle_{\Lambda_{N}, h} & =\liminf _{N \rightarrow \infty}\left\langle\frac{S_{N}}{N}\right\rangle_{\Lambda_{N}, h}=\liminf _{N \rightarrow \infty} \int_{\Omega_{\Lambda}} \frac{S_{N}(\sigma)}{N} \mathbb{P}_{\Lambda_{N}, \beta, h}(\sigma) d \sigma \\
& +\liminf _{N \rightarrow \infty} \int_{\left|\frac{S_{N}}{N}\right| \leq a} \frac{S_{N}(\sigma)}{N} \mathbb{P}_{\Lambda_{N}, \beta, h}(\sigma) d \sigma \\
& \geq-a+\liminf _{N \rightarrow \infty} \int_{\frac{S_{N}}{N}>+a} \frac{S_{N}(\sigma)}{N} \mathbb{P}_{\Lambda_{N}, \beta, h}(\sigma) d \sigma \\
& \geq-a+m^{(2)}-a^{2} .
\end{aligned}
$$

Contudo, como o resultado é válido para todo $a \in(0,1]$, segue

$$
m(\beta, h)=\lim _{N \rightarrow \infty}\left\langle\frac{S_{N}}{N}\right\rangle_{\Lambda_{N}, h} \geq m^{(2)}
$$

Com uma pequena adaptação do argumento dado acima, temos na verdade provado o seguinte lema.

Lema 2.5.1. Dado um modelo de Ising finito definido em uma caixa simétrica $\Lambda_{N}=\mathbb{Z} \cap[-N,+N]$, com constantes de acoplamento $J_{i j} \in \mathbb{R}$ (não necessariamente ferromagnéticas), campo magnético $h_{i}$ tal que $\liminf _{n \rightarrow \infty} h_{i} \geq$ $h>0$ e condições exteriores livres, fixo qualquer valor do inverso da temperatura $\beta$, se existir o limite das magnetizações finitas

$$
m\left(\beta,\left\{h_{i}\right\}_{i \in \mathbb{Z}}\right)=\lim _{N \rightarrow \infty}\left\langle\frac{S_{N}}{\left|\Lambda_{N}\right|}\right\rangle_{\Lambda_{N},\left\{h_{i}\right\}_{i \in \Lambda_{N}}}
$$

$\boldsymbol{e} \liminf \operatorname{in}_{N \rightarrow \infty}\left\langle\left(S_{N} /\left|\Lambda_{N}\right|\right)^{2}\right\rangle_{\Lambda_{N},\left\{h_{i}\right\}_{i \in \Lambda_{N}}} \geq m^{(2)}\left(\beta,\left\{h_{i}\right\}_{i \in \mathbb{Z}}\right)>0$, então $m\left(\beta,\left\{h_{i}\right\}_{i \in \mathbb{Z}}\right) \geq$ $m^{(2)}\left(\beta,\left\{h_{i}\right\}_{i \in \mathbb{Z}}\right)>0$. 


\section{Capítulo 3}

\section{Modelos com Interações Translacionalmente Invariante}

\subsection{Introdução}

Nos capítulos anteriores definimos o modelo de Ising com interações ferromagnéticas $J_{i j}$. Em alguns dos resultados expostos a única propriedade importante das interações $J_{i j}$ era sua positividade. O tema deste capítulo são os sistema com constantes de acoplamento $J_{i j}$ dependente apenas da distância entre os sítio $i$ e $j$, ou seja, $J_{i j} \equiv J(|i-j|)$.

Na Seção 3.2 apresentamos o modelo de Curie-Weiss (CW), modelo no qual cada spin interage apenas com a magnetização total. Por estar entre os mais simples modelos de Ising que apresentam transição de fase, é possível realizar uma série de cálculos explicitos envolvendo esse modelo.

A Seção 3.3 traz os cálculos detalhados de uma função denomida pressão nesse modelo. Em seguida, na Seção 3.4 é apresentada a relação entre a derivada da pressão e a magnetização. Continuando o raciocínio, a Seção 3.5 utiliza os resultados anteriores para calcular explicitamente a magnetização no modelo de CW, mostrando que há uma região de altas temperaturas em que a magnetização é nula. Finalizando a análise do modelo de CW, a Seção 3.6 compara um Modelo de Ising arbitrário (somável) com um modelo de CW, mostrando que este último apresenta magnetização que não supera a do primeiro. Essa linha de raciocínio demonstra que qualquer Modelo de Ising somável apresenta uma temperatura crítica finita acima da qual a magnetização é nula.

Em 3.7, tratamos dos modelos com interação do tipo lei de potência, isto é, $J_{i j}=|i-j|^{-\alpha}$. Nessa seção citamos vários resultados já conhecidos 
em 1969 sobre esses modelos. Veremos que no limite quando $N \rightarrow \infty$, para o caso não somável $\alpha \leq 1$, quase todos os spins estarão alinhados com probabilidade 1 e haverá magnetização não nula para todo $\beta$. Já para o caso $\alpha>2$, a situação é inversa para todo $\beta>0$ a magnetização limite será zero. Os casos mais interessantes, que apresentam transição de fase, serão tratados no próximo capítulo.

A última seção do capítulo, 3.8 mostra como ao se classificar os modelos do tipo lei de potência ganhamos também maior entendimento sobre outros modelos com interações translacionalmente invariantes.

\subsection{Modelo de Curie-Weiss}

O modelo de Curie-Weiss a volume finito é um modelo de Ising como apresentado na Seção 1.3 em que as constantes de acoplamento $J(|i-j|)$ são dadas por $J_{i j}=\mathscr{J} /|\Lambda|$, sendo $\mathscr{J}$ uma constante real positiva. Há uma importante diferença na sequência de modelos que tomamos ao calcular funções no Limite Termodinâmico. No modelo de Ising dado um par de spins $(i, j)$ as constantes de acoplamento $J_{i j}$ são fixas, independentes do tamanho da caixa $|\Lambda|$. Já no modelo de Curie-Weiss essas constantes de acoplamento variam com o volume $|\Lambda|$, sendo constante apenas o parâmetro $\mathscr{J}$, correspondente à soma das interações de um spin com os demais.

Neste texto, vamos nos concentrar exclusivamente no caso ferromagnético, isto é, $\mathscr{J} \geq 0$. O caso $\mathscr{J}=0$ é trivial, pois a medida de Gibbs é na verdade a medida produto de medidas de Bernoulli. A energia de cada estado $\sigma$ nesse modelo será denotada por $H_{\Lambda, h}^{C W}(\sigma)$ e dada por:

$$
H_{\Lambda, h}^{C W}(\sigma)=-\sum_{i, j \in \Lambda} \frac{\mathscr{J}}{2|\Lambda|} \sigma_{i} \sigma_{j}-\sum_{i \in \Lambda} h \sigma_{i}
$$

Se $h=0$, por exemplo, então temos que

$$
H_{\Lambda, 0}^{C W}(\sigma)=-\sum_{i, j \in \Lambda} \frac{\mathscr{J}}{2|\Lambda|} \sigma_{i} \sigma_{j}=-\sum_{i \in \Lambda} \sigma_{i} \frac{\mathscr{J}}{2}\left[\sum_{j \in \Lambda} \frac{\sigma_{j}}{|\Lambda|}\right]=-\sum_{i \in \Lambda} \frac{\mathscr{J}}{2} \sigma_{i}\left[\frac{S_{\Lambda}}{|\Lambda|}\right] .
$$

No modelo de Curie-Weiss é simples entender a relação entre o valor esperado de um spin qualquer $\left\langle\sigma_{i}\right\rangle_{\Lambda}$ e a magnetização específica $m_{\Lambda, h}=$ $\left\langle S_{\Lambda} /|\Lambda|\right\rangle$. Para isso basta observar que devido à simetria do modelo, dados dois sítios $i$ e $j$ quaisquer, temos $\left\langle\sigma_{i}\right\rangle_{\Lambda, h}=\left\langle\sigma_{j}\right\rangle_{\Lambda, h}$. Logo, segue da linearidade 
do valor esperado, que

$$
m_{\Lambda, h}=\left\langle\frac{S_{\Lambda}}{|\Lambda|}\right\rangle_{\Lambda, h}=\left\langle\frac{\sum_{i} \sigma_{i}}{|\Lambda|}\right\rangle_{\Lambda, h}=\frac{1}{|\Lambda|} \sum_{i}\left\langle\sigma_{i}\right\rangle_{\Lambda, h}=\frac{1}{|\Lambda|}|\Lambda|\left\langle\sigma_{i}\right\rangle_{\Lambda, h}=\left\langle\sigma_{i}\right\rangle_{\Lambda, h}
$$

ou seja, o valor esperado de um spin qualquer é exatamente a magnetização específica.

\subsection{A Pressão do Modelo de Curie-Weiss}

Nesta seção vamos definir e calcular a pressão do modelo de Curie-Weiss. A função de partição $Z_{\Lambda}$ desse modelo pode ser calculada utilizando o hamiltoniano (3.1). Para cada natural $N$, temos $\Lambda=[-N, N] \cap \mathbb{Z} \mathbf{e}$

$$
\begin{aligned}
Z_{\Lambda} & =\sum_{\sigma \in \Omega_{\Lambda}} \exp \left(\sum_{i, j \in \Lambda} \frac{\beta \mathscr{J}}{2|\Lambda|} \sigma_{i} \sigma_{j}+\beta h \sum_{i \in \Lambda} \sigma_{i}\right) \\
& =\sum_{\sigma \in \Omega_{\Lambda}} \exp \left(\frac{\beta \mathscr{J}|\Lambda|}{2}\left(\sum_{i \in \Lambda} \frac{\sigma_{i}}{|\Lambda|}\right)^{2}+\beta h \sum_{i \in \Lambda} \sigma_{i}\right) .
\end{aligned}
$$

Claramente o termo quadrático na expressão acima é o termo que cria dificuldade na obtenção de uma fórmula mais explícita para $Z_{\Lambda}$ e consequentemente para a pressão, cuja definição será apresentada mais a frente. Para superar essa dificuldade, a ideia é reescrever o termo quadrático como uma integral Gaussiana. O primeiro passo para isso é considerar a seguinte identidade:

$$
e^{a^{2}}=\frac{1}{\sqrt{2 \pi}} \int_{\mathbb{R}} e^{\frac{-t^{2}}{2}+\sqrt{2} a t} d t
$$

que é válida para todo $a \in \mathbb{R}$. Usando essa identidade na expressão da função de partição com

$$
a=\sqrt{\frac{\beta \mathscr{J}|\Lambda|}{2}} \sum_{i \in \Lambda} \frac{\sigma_{i}}{|\Lambda|}
$$


temos que

$$
\begin{aligned}
Z_{\Lambda} & =\sum_{\sigma \in \Omega_{\Lambda}} \exp \left(\frac{\beta \mathscr{J}|\Lambda|}{2}\left(\sum_{i \in \Lambda} \frac{\sigma_{i}}{|\Lambda|}\right)^{2}\right) \exp \left(\beta h \sum_{i \in \Lambda} \sigma_{i}\right) \\
& =\sum_{\sigma \in \Omega_{\Lambda}} \exp \left(\left(\sqrt{\frac{\beta \mathscr{J}|\Lambda|}{2}} \sum_{i \in \Lambda} \frac{\sigma_{i}}{|\Lambda|}\right)^{2}\right) \exp \left(\beta h \sum_{i \in \Lambda} \sigma_{i}\right) \\
& =\frac{1}{\sqrt{2 \pi}} \sum_{\sigma \in \Omega_{\Lambda}} \int_{\mathbb{R}} \exp \left(\frac{-t^{2}}{2}+t \sqrt{\frac{\beta \mathscr{J}}{|\Lambda|}} \sum_{i \in \Lambda} \sigma_{i}\right) d t \cdot \exp \left(\beta h \sum_{i \in \Lambda} \sigma_{i}\right) \\
& =\frac{1}{\sqrt{2 \pi}} \sum_{\sigma \in \Omega_{\Lambda}} \int_{\mathbb{R}} \prod_{i \in \Lambda}\left[\exp \left(t \sqrt{\frac{\beta \mathscr{J}}{|\Lambda|}} \sigma_{i}+\beta h \sigma_{i}\right)\right] \exp \left(\frac{-t^{2}}{2}\right) d t .
\end{aligned}
$$

Para simplificar o termo que aparece mais à direita da igualdade acima vamos precisar da seguinte observação: se $G: \mathbb{R}^{2} \rightarrow \mathbb{R}$ é uma função Borel mensurável não-negativa, então

$$
\begin{aligned}
\sum_{\sigma \in \Omega_{\Lambda}} \int_{\mathbb{R}} \prod_{i \in \Lambda} G\left(t, \sigma_{i}\right) d t & =\sum_{\sigma_{-N} \in\{-1,1\}} \ldots \sum_{\sigma_{N} \in\{-1,1\}} \int_{\mathbb{R}} \prod_{i \in \Lambda} G\left(t, \sigma_{i}\right) d t \\
& =\int_{\mathbb{R}}\left[\sum_{\sigma_{-N} \in\{-1,1\}} \ldots \sum_{\sigma_{N} \in\{-1,1\}} G\left(t, \sigma_{-N}\right) \ldots G\left(t, \sigma_{N}\right)\right] d t \\
& =\int_{\mathbb{R}}\left[\sum_{\sigma_{-N} \in\{-1,1\}} G\left(t, \sigma_{N}\right) \quad \ldots \sum_{\sigma_{N} \in\{-1,1\}} G\left(t, \sigma_{N}\right)\right] d t \\
& =\int_{\mathbb{R}}\left[\prod_{i \in \Lambda} \sum_{\sigma_{i} \in\{-1,1\}} G\left(t, \sigma_{i}\right)\right] d t .
\end{aligned}
$$

Em vista dessa observação e de (3.3) obtemos

$$
Z_{\Lambda}=\frac{1}{\sqrt{2 \pi}} \int_{\mathbb{R}} \prod_{i \in \Lambda}\left[\sum_{\sigma_{i} \in\{-1,1\}} \exp \left(t \sqrt{\frac{\beta \mathscr{J}}{|\Lambda|}} \sigma_{i}+\beta h \sigma_{i}\right)\right] \exp \left(\frac{-t^{2}}{2}\right) d t .
$$

A expressão dentro do produtório acima pode ser escrita como

$$
\sum_{\sigma_{i} \in\{-1,1\}} \exp \left(t \sqrt{\frac{\beta \mathscr{J}}{|\Lambda|}} \sigma_{i}+\beta h \sigma_{i}\right)=2 \cosh \left(t \sqrt{\frac{\beta \mathscr{J}}{|\Lambda|}}+\beta h\right) .
$$


Substituindo essa expressão na última igualdade obtida para a função de partição ficamos com

$$
\begin{aligned}
Z_{\Lambda} & =\frac{1}{\sqrt{2 \pi}} \int_{\mathbb{R}} \prod_{i \in \Lambda}\left[2 \cosh \left(t \sqrt{\frac{\beta \mathscr{J}}{|\Lambda|}}+\beta h\right)\right] \exp \left(\frac{-t^{2}}{2}\right) d t \\
& =\frac{2^{|\Lambda|}}{\sqrt{2 \pi}} \int_{\mathbb{R}}\left[\cosh \left(t \sqrt{\frac{\beta \mathscr{J}}{|\Lambda|}}+\beta h\right)\right]^{|\Lambda|} \exp \left(\frac{-t^{2}}{2}\right) d t .
\end{aligned}
$$

Com essa expressão alternativa da função de partição, é interessante neste ponto definir a pressão.

Definição 3.3.1. Considere a sequência de volumes $\Lambda_{N}=[-N, N] \cap \mathbb{Z}$. A pressão do modelo de Curie-Weiss é definida por

$$
p(\beta, h)=\lim _{N \rightarrow \infty} \frac{1}{\left|\Lambda_{N}\right|} \log Z_{\Lambda_{N}}(\beta, h) .
$$

Observação 3.3.1. A prova de que $p(\beta, h)$ está bem definida para todo $\beta>0$ e $h \in \mathbb{R}$ (existência do limite acima) será dada na sequência, usando a representação integral que obtivemos em (3.4) para a função de partição $Z_{\Lambda}$. Além de mostrar a existência de tal limite, vamos também obter uma fórmula explicita para a pressão, veja Teorema 3.3 .3

Para provar a existência do limite acima vamos precisar do seguinte lema auxiliar.

Lema 3.3.2. Seja $(\Omega, \mathcal{F}, \mu)$ um espaço de medida. Suponha que para todo $1 \leq p \leq \infty$ temos $f \in L^{p}(\Omega, \mathcal{F}, \mu)$. Então $\|f\|_{p} \rightarrow\|f\|_{\infty}$, quando $p \rightarrow \infty$.

Demonstração. Seja $K_{\delta}=\left\{\omega \in \Omega:\left(\|f\|_{\infty}-\delta\right) \leq|f(\omega)|\right\}$. Então temos que $1_{K_{\delta}}\left(\|f\|_{\infty}-\delta\right)^{p} \leq 1_{K_{\delta}}|f|^{p}$. Integrando ambos os lados dessa desigualdade com respeito a $\mu$ temos que

$$
\mu\left(K_{\delta}\right)\left(\|f\|_{\infty}-\delta\right)^{p} \leq \int_{\Omega} 1_{K_{\delta}}|f|^{p} d \mu \leq\|f\|_{p}^{p} .
$$

Tomando a raíz $p$-ésima nessa desigualdade e em seguida o lim inf quando $p \rightarrow \infty$ ficamos com

$$
\left(\|f\|_{\infty}-\delta\right)=\liminf _{p \rightarrow \infty} \mu\left(K_{\delta}\right)^{\frac{1}{p}}\left(\|f\|_{\infty}-\delta\right) \leq \liminf _{p \rightarrow \infty}\|f\|_{p} .
$$


Já que $\delta>0$ é arbitrário segue que

$$
\|f\|_{\infty} \leq \liminf _{p \rightarrow \infty}\|f\|_{p} .
$$

Como $|f(\omega)| \leq\|f\|_{\infty} \mu$-quase certamente temos que para todo $1 \leq q<p$ que

$$
\|f\|_{p}^{p}=\int_{\Omega}|f(\omega)|^{p-q}|f(\omega)|^{q} d \mu(\omega) \leq\|f\|_{\infty}^{p-q} \int_{\Omega}|f(\omega)|^{q} d \mu(\omega)=\|f\|_{\infty}^{p-q}\|f\|_{q}^{q} .
$$

Tomando a raíz $p$-ésima em ambos lados da desigualdade acima e em seguida o limsup quando $p \rightarrow \infty$ ficamos com

$$
\limsup _{p \rightarrow \infty}\|f\|_{p} \leq \limsup _{p \rightarrow \infty}\left(\|f\|_{\infty}^{\frac{p-q}{p}}\|f\|_{q}^{\frac{q}{p}}\right)=\limsup _{p \rightarrow \infty}\left(\|f\|_{\infty}^{\frac{p-q}{p}}\right) \limsup _{p \rightarrow \infty}\left(\|f\|_{q}^{\frac{q}{p}}\right)=\|f\|_{\infty} .
$$

A desigualdade acima juntamente com (3.5) nos fornece

$$
\limsup _{p \rightarrow \infty}\|f\|_{p} \leq\|f\|_{\infty} \leq \liminf _{p \rightarrow \infty}\|f\|_{p}
$$

e, portanto,

$$
\|f\|_{\infty}=\lim _{p \rightarrow \infty}\|f\|_{p}
$$

Teorema 3.3.3. Sejam $N \in \mathbb{N}$ e $\Lambda_{N}=[-N, N] \cap \mathbb{Z}$. Se $\mathscr{J}, \beta>0$ e $h \in \mathbb{R}$ então a pressão do modelo de Curie-Weiss é dada por

$$
p(\beta, h) \equiv \lim _{N \rightarrow \infty} \frac{\log Z_{\Lambda_{N}}}{\left|\Lambda_{N}\right|}=\log (2)+\sup _{x \in \mathbb{R}}\left\{\log (\cosh (x))-\frac{(x-\beta h)^{2}}{2 \beta \mathscr{J}}\right\} .
$$

Demonstração. Para facilitar a notação vamos omitir o subíndice $N$ no volume $\Lambda_{N}$, escrevendo apenas $\Lambda$ e também escreveremos $Z_{\Lambda}$ ao invés de $\log Z_{\Lambda_{N}}(\beta, h)$. Pelas propriedades básicas do logaritmo e por (3.4) temos que $(1 /|\Lambda|) \log Z_{\Lambda}=\log Z_{\Lambda}^{\frac{1}{\Lambda \mid}}$ e também igual a

$$
\begin{array}{r}
\log \left(\frac{2}{(2 \pi)^{\frac{1}{2|\Lambda|}}}\left[\int_{\mathbb{R}}\left[\cosh \left(t \sqrt{\frac{\beta \mathscr{J}}{|\Lambda|}}+\beta h\right)\right]^{|\Lambda|} \exp \left(\frac{-t^{2}}{2}\right) d t\right]^{\frac{1}{|\Lambda|}}\right) \\
=\log \left(\frac{2}{(2 \pi)^{\frac{1}{2|\Lambda|}}}\right)+\log \left(\left[\int_{\mathbb{R}}\left[\cosh \left(t \sqrt{\frac{\beta \mathscr{J}}{|\Lambda|}}+\beta h\right)\right]^{|\Lambda|} \exp \left(\frac{-t^{2}}{2}\right) d t\right]^{\frac{1}{|\Lambda|}}\right)
\end{array}
$$

É fácil ver que a primeira parcela no lado direito da igualdade acima con- 
verge para $\log (2)$ quando $|\Lambda| \rightarrow \infty$. Precisamos mostrar que a segunda parcela também converge. Fazendo na integral acima a seguinte mudança de variáveis

$$
u=t \sqrt{\frac{\beta \mathscr{J}}{|\Lambda|}}+\beta h \Longrightarrow t=(u-\beta h) \sqrt{\frac{|\Lambda|}{\beta \mathscr{J}}} \quad \mathrm{e} \quad d t=\sqrt{\frac{|\Lambda|}{\beta \mathscr{J}}} d u .
$$

o segundo logaritmo acima pode ser reescrito como

$$
\log \left(\left(\frac{|\Lambda|}{\beta \mathscr{J}}\right)^{\frac{1}{2|\Lambda|}}\left[\int_{\mathbb{R}}[\cosh (u)]^{|\Lambda|} \exp \left(\frac{-(u-\beta h)^{2}|\Lambda|}{2 \beta \mathscr{J}}\right) d u\right]^{\frac{1}{|\Lambda|}}\right) .
$$

Usando propriedades básicas do logaritmo e fazendo apenas uma simplificação algébrica no integrando, a expressão se torna

$$
\log \left(\left(\frac{|\Lambda|}{\beta \mathscr{J}}\right)^{\frac{1}{2|\Lambda|}}\right)+\log \left(\left[\int_{\mathbb{R}}\left[\cosh (u) \cdot \exp \left(\frac{-(u-\beta h)^{2}}{2 \beta \mathscr{J}}\right)\right]^{|\Lambda|} d u\right]^{\frac{1}{|\Lambda|}}\right) .
$$

Já que para todo $p \geq 1$ a função $F$ dada

$$
F(u) \equiv \cosh (u) \cdot \exp \left(\frac{-(u-\beta h)^{2}}{2 \beta \mathscr{J}}\right)
$$

pertence a $L^{p}(\mathbb{R}, \mathscr{B}(\mathbb{R}), d x$ ) podemos aplicar o Lema (3.3.2) para garantir que o argumento do segundo logaritmo acima converge para $\|F\|_{\infty}>0$, quando $|\Lambda| \rightarrow \infty$. Já que

$$
\frac{1}{|\Lambda|} \log Z_{\Lambda}=\log \left(\frac{2}{(2 \pi)^{\frac{1}{2|\Lambda|}}}\right)+\log \left(\left(\frac{|\Lambda|}{\beta \mathscr{J}}\right)^{\frac{1}{2|\Lambda|}}\right)+\log \left(\|F\|_{|\Lambda|}\right)
$$

o lado direito é soma de três sequências convergentes e, portanto, converge. Além disso, o limite é dado por

$$
\begin{aligned}
p(\beta, h) \equiv \lim _{|\Lambda| \rightarrow \infty} \frac{1}{|\Lambda|} \log Z_{\Lambda} & =\log (2)+\log \left(\|F\|_{\infty}\right) \\
& =\log (2)+\log \left(\sup _{x \in \mathbb{R}}\left\{\cosh (u) \cdot \exp \left(\frac{-(u-\beta h)^{2}}{2 \beta \mathscr{J}}\right)\right\}\right) \\
& =\log (2)+\sup _{x \in \mathbb{R}}\left\{\log (\cosh (x))-\frac{(x-\beta h)^{2}}{2 \beta \mathscr{J}}\right\} .
\end{aligned}
$$


Estamos interessados na pressão do modelo de Curie-Weiss porque podemos mostrar que a magnetização específica desse modelo está relacionada com uma derivada parcial da pressão. De maneira mais precisa, vamos mostrar na seção seguinte que a função $f(\beta, h)=-\beta^{-1} p(\beta, h)$, (chamada de Energia Livre) para todo $h \neq 0$ e $\beta>0$, tem derivada parcial com relação a $h$, que a sequência $m_{\Lambda, h} \rightarrow m(\beta, h)$ de fato converge para todo $\beta>0$ e $h \in \mathbb{R}$, e, além disso,

$$
-\frac{\partial f}{\partial h}(\beta, h)=m(\beta, h) \equiv \lim _{\Lambda \rightarrow \mathbb{Z}} m_{\Lambda, h} .
$$

Como temos uma fórmula explícita para $p$ e, consequentemente, para $f$, poderemos mostrar, usando a equação acima, que não há magnetização espontânea para $\beta \in\left(0, \mathscr{J}^{-1}\right]$. Em outras palavras, vamos mostrar que se $\beta \in\left(0, \mathscr{J}^{-1}\right]$ então

$$
\lim _{h \rightarrow 0^{+}} m(\beta, h) \equiv \lim _{h \rightarrow 0^{+}} \lim _{\Lambda \rightarrow \mathbb{Z}} m_{\Lambda, h}=\lim _{h \rightarrow 0^{+}}-\frac{\partial f}{\partial h}(\beta, h)=0 .
$$

Por outro lado, se $\beta>\mathscr{J}^{-1}$ então mostraremos que há magnetização espontânea, isto é,

$$
\lim _{h \rightarrow 0^{+}} m(\beta, h)=\lim _{h \rightarrow 0^{+}}-\frac{\partial f}{\partial h}(\beta, h)>0 .
$$

Portanto, no modelo de Curie-Weiss sabemos exatamente qual é o inverso da temperatura crítica de transição de fase, $\beta_{c}=\mathscr{J}^{-1}$ !

No capítulo anterior mostramos que $m_{\Lambda, h}=-m_{\Lambda,-h}$. Usando essa identidade em (3.6) temos que

$$
-\frac{\partial f}{\partial h}(\beta, h)=\frac{\partial f}{\partial h}(\beta,-h) .
$$

Dessa forma, no modelo de Curie-Weiss se $\beta>\mathscr{J}^{-1}$ (onde o modelo tem magnetização espontânea) então

$$
\lim _{h \rightarrow 0^{+}}-\frac{\partial f}{\partial h}(\beta, h)=\lim _{h \rightarrow 0^{+}} m(\beta, h)>0 \quad \text { e } \quad \lim _{h \rightarrow 0^{-}}-\frac{\partial f}{\partial h}(\beta, h)=-\lim _{h \rightarrow 0^{-}} m(\beta,-h)<0 .
$$

Logo a Energia Livre (ou pressão) tem derivada parcial com respeito a $h$ descontínua nos pontos em que $h=0$ e $\beta>\mathscr{J}^{-1}$. Dito de outra forma, para uma temperatura fixa no regime onde ocorre magnetização espontênea (baixas temperaturas) a energia livre vista como função do campo externo $h$ é descontínua na origem $h=0$. 


\subsection{Curie-Weiss e a Derivada da Pressão}

Nesta seção vamos definir a pressão e a energia livre a volume finito do modelo de Curie-Weiss e estudar suas relações com a magnetização específica a volume $\Lambda$.

A Pressão e a energia livre a volume $\Lambda=[-N, N] \cap \mathbb{Z}$ são definidas para $\beta>0$ e $h \in \mathbb{R}$ pelas seguintes expressões

$$
p_{\Lambda}(\beta, h) \equiv \frac{\log Z_{\Lambda_{N}}(\beta, h)}{\left|\Lambda_{N}\right|} \quad \text { e } \quad f_{\Lambda}(\beta, h)=-\frac{1}{\beta} p_{\Lambda}(\beta, h) .
$$

Teorema 3.4.1 (Convexidade de Pressão). Sejam $N \in \mathbb{N}$ e $\Lambda=[-N, N] \cap \mathbb{Z}$. Fixado $\beta>0$, temos que a função $h \mapsto p_{\Lambda}(\beta, h)$ é uma função convexa de $\mathbb{R}$ em $\mathbb{R}$.

Demonstração. Para mostrar que, para qualquer $\beta$ fixado, $h \mapsto p_{\Lambda}(\beta, h)$ é uma função convexa é suficiente mostrar para todo $0 \leq \alpha \leq 1$ e $h_{1}, h_{2} \in \mathbb{R}$ vale a seguinte desigualdade

$$
Z_{\Lambda}\left(\beta, \alpha h_{1}+(1-\alpha) h_{2}\right) \leq Z_{\Lambda}\left(\beta, h_{1}\right)^{\alpha} \cdot Z_{\Lambda}\left(\beta, h_{2}\right)^{1-\alpha} .
$$

De fato, tomando o logaritmo em ambos os lados da dessa desigualdade e em seguida dividindo por $|\Lambda|$ obtemos

$$
\begin{aligned}
p_{\Lambda}\left(\beta, \alpha h_{1}+(1-\alpha) h_{2}\right) & \equiv \frac{1}{|\Lambda|} \log Z_{\Lambda}\left(\beta, \alpha h_{1}+(1-\alpha) h_{2}\right) \\
& \leq \frac{\alpha}{|\Lambda|} \log Z_{\Lambda}\left(\beta, h_{1}\right)+\frac{1-\alpha}{|\Lambda|} \log Z_{\Lambda}\left(\beta, h_{2}\right) \\
& =\alpha p_{\Lambda}\left(\beta, h_{1}\right)+(1-\alpha) p_{\Lambda}\left(\beta, h_{2}\right) .
\end{aligned}
$$

Assim, tudo que temos que fazer é mostrar que a desigualdade (3.7) é válida sob as hipóteses do teorema. Pela definição da função de partição o lado esquerdo de (3.7) é dado por

$$
\sum_{\sigma \in \Omega_{\Lambda}} \exp \left(\sum_{i, j \in \Lambda} \frac{\beta \mathscr{J}}{2|\Lambda|} \sigma_{i} \sigma_{j}+\beta\left[\alpha h_{1}+(1-\alpha) h_{2}\right] \sum_{i \in \Lambda} \sigma_{i}\right)
$$

que por sua vez é igual a

$$
\sum_{\sigma \in \Omega_{\Lambda}} \exp \left(\sum_{i, j \in \Lambda} \frac{\beta \mathscr{J}}{2|\Lambda|} \sigma_{i} \sigma_{j}\right) \cdot \exp \left(\alpha \beta h_{1} \sum_{i \in \Lambda} \sigma_{i}\right) \exp \left((1-\alpha) \beta h_{2} \sum_{i \in \Lambda} \sigma_{i}\right) .
$$


Usando as propriedades básicas da função exponencial temos que a expressão acima é igual a

$$
\sum_{\sigma \in \Omega_{\Lambda}} \exp \left(\sum_{i, j \in \Lambda} \frac{\beta \mathscr{J}}{2|\Lambda|} \sigma_{i} \sigma_{j}\right) \cdot\left[\exp \left(\beta h_{1} \sum_{i \in \Lambda} \sigma_{i}\right)\right]^{\alpha}\left[\exp \left(\beta h_{2} \sum_{i \in \Lambda} \sigma_{i}\right)\right]^{1-\alpha}
$$

Considerando a medida $\mu$ definida no conjunto das partes de $\Omega_{\Lambda}$ por

$$
\mu(E)=\int_{\Omega_{\Lambda}} 1_{E}(\sigma) \exp \left(\sum_{i, j \in \Lambda} \frac{\beta \mathscr{J}}{2|\Lambda|} \sigma_{i} \sigma_{j}\right) d \kappa(\sigma),
$$

onde $\kappa$ é a medida da contagem em $\Omega_{\Lambda}$, podemos reescrever a expressão (3.10), que é igual a $Z_{\Lambda}\left(\beta, \alpha h_{1}+(1-\alpha) h_{2}\right)$, como segue

$$
Z_{\Lambda}\left(\beta, \alpha h_{1}+(1-\alpha) h_{2}\right)=\int_{\Omega_{\Lambda}}\left[\exp \left(\beta h_{1} \sum_{i \in \Lambda} \sigma_{i}\right)\right]^{\alpha}\left[\exp \left(\beta h_{2} \sum_{i \in \Lambda} \sigma_{i}\right)\right]^{1-\alpha} d \mu(\sigma) .
$$

Aplicando a desigualdade de Hölder no lado direito da igualdade acima $\operatorname{com} p=1 / \alpha$ e $q=1 /(1-\alpha)$ obtemos

$$
\begin{aligned}
Z_{\Lambda}(\beta, \alpha & \left.h_{1}+(1-\alpha) h_{2}\right)=\int_{\Omega_{\Lambda}}\left[\exp \left(\beta h_{1} \sum_{i \in \Lambda} \sigma_{i}\right)\right]^{\alpha}\left[\exp \left(\beta h_{2} \sum_{i \in \Lambda} \sigma_{i}\right)\right]^{1-\alpha} d \mu(\sigma) \\
\leq & \left(\int_{\Omega_{\Lambda}}\left[\exp \left(\beta h_{1} \sum_{i \in \Lambda} \sigma_{i}\right)\right] d \mu(\sigma)\right)^{\alpha}\left(\int_{\Omega_{\Lambda}}\left[\exp \left(\beta h_{2} \sum_{i \in \Lambda} \sigma_{i}\right)\right] d \mu(\sigma)\right)^{1-\alpha} \\
& \leq Z_{\Lambda}\left(\beta, h_{1}\right)^{\alpha} Z_{\Lambda}\left(\beta, h_{2}\right)^{1-\alpha} .
\end{aligned}
$$

Provando (3.7) e, consequentemente, a convexidade de $h \mapsto p_{\Lambda}(\beta, h)$.

Corolário 3.4.2. Sejam $N \in \mathbb{N}$ e $\Lambda=[-N, N] \cap \mathbb{Z}$. Fixado $\beta>0$ temos que a função $h \mapsto f_{\Lambda}(\beta, h)$ é uma função concava de $\mathbb{R}$ em $\mathbb{R}$.

Demonstração. Já que $f_{\Lambda}(\beta, h)=-\beta^{-1} p_{\Lambda}(\beta, h)$ e $\beta>0$ é uma constante o resultado segue diretamente da convexidade de $h \mapsto p_{\Lambda}(\beta, h)$.

Teorema 3.4.3 (Concavidade da Magnetização). Sejam $N \in \mathbb{N} e \Lambda=$ $[-N, N] \cap \mathbb{Z}$. Fixados $\mathscr{J}$ e $\beta>0$, temos que a função $h \mapsto m_{\Lambda}(\beta, h)$ é uma função côncava em $[0,+\infty)$.

Demonstração. Como para todo $\Lambda$ e $\beta>0$ fixados $h \mapsto m_{\Lambda}(\beta, h)$ é uma função de classe $C^{\infty}$ é suficiente provar que $\frac{d^{2}}{d h^{2}} m_{\Lambda}(\beta, h) \leq 0$. Do Corolário 2.2 .3 
temos

$$
\frac{\partial}{\partial h_{i}} m_{\Lambda, \beta,\left\{h_{i}\right\}_{i \in \Lambda}}=\frac{1}{|\Lambda|} \beta \sum_{j \in \Lambda}\left[\left\langle\sigma_{j} \sigma_{i}\right\rangle_{\Lambda, \beta,\left\{h_{i}\right\}_{i \in \Lambda}}-\left\langle\sigma_{j}\right\rangle_{\Lambda, \beta,\left\{h_{i}\right\}_{i \in \Lambda}}\left\langle\sigma_{i}\right\rangle_{\Lambda, \beta,\left\{h_{i}\right\}_{i \in \Lambda}}\right]
$$

Considere a aplicação $\gamma: \mathbb{R} \rightarrow \mathbb{R}^{2 N+1}$ dada por $\gamma(h)=(h, \ldots, h)$. Então temos para todo $h \in \mathbb{R}$ que a magnetização específica do modelo de Curie-Weiss com campo constante $m_{\Lambda, \beta, h}$ é dada por $m_{\Lambda, \beta,\left\{h_{i}\right\}_{i \in \Lambda}}$, com $h_{i}=h, \forall i \in \Lambda$. A regra cadeia nos dá

$$
\begin{aligned}
\frac{d}{d h} m_{\Lambda, \beta, h} & =\left.\nabla m_{\Lambda, \beta,\left\{h_{i}\right\}_{i \in \Lambda}}\right|_{\left\{h_{i}\right\}_{i \in \Lambda}=\gamma(h)} \cdot \gamma^{\prime}(h) \\
& =\sum_{i \in \Lambda} \frac{\partial}{\partial h_{i}} m_{\Lambda, \beta,\left\{h_{i}\right\}_{i \in \Lambda}} \\
& =\frac{1}{|\Lambda|} \beta \sum_{i, j \in \Lambda}\left[\left\langle\sigma_{j} \sigma_{i}\right\rangle_{\Lambda, \beta, h}-\left\langle\sigma_{j}\right\rangle_{\Lambda, \beta, h}\left\langle\sigma_{i}\right\rangle_{\Lambda, \beta, h}\right]
\end{aligned}
$$

Note que, nessa última igualdade, o valor esperado é calculado com campo externo constante igual a $h$. Um cálculo análogo ao feito na prova do Corolário 2.2.3 mostra que

$$
\frac{d}{d h}\left\langle\sigma_{j} \sigma_{i}\right\rangle_{\Lambda, \beta, h}=\beta \sum_{k \in \Lambda}\left[\left\langle\sigma_{i} \sigma_{j} \sigma_{k}\right\rangle_{\Lambda, \beta, h}-\left\langle\sigma_{i} \sigma_{j}\right\rangle_{\Lambda, \beta, h}\left\langle\sigma_{k}\right\rangle_{\Lambda, \beta, h}\right]
$$

Uma vez que sabemos calcular

$$
\frac{d}{d h}\left\langle\sigma_{i}\right\rangle_{\Lambda, \beta, h}=\beta \sum_{k \in \Lambda}\left[\left\langle\sigma_{i} \sigma_{k}\right\rangle_{\Lambda, \beta, h}-\left\langle\sigma_{i}\right\rangle_{\Lambda, \beta, h}\left\langle\sigma_{k}\right\rangle_{\Lambda, \beta, h}\right]
$$

temos, pela regra do produto para derivadas, que

$$
\begin{aligned}
& \frac{d^{2}}{d h^{2}} m_{\Lambda, \beta, h}= \frac{\beta}{|\Lambda|} \sum_{i, j \in \Lambda}\left[\frac{d}{d h}\left\langle\sigma_{j} \sigma_{i}\right\rangle_{\Lambda, \beta, h}-\frac{d}{d h}\left\langle\sigma_{j}\right\rangle_{\Lambda, \beta, h}\left\langle\sigma_{i}\right\rangle_{\Lambda, \beta, h}-\left\langle\sigma_{j}\right\rangle_{\Lambda, \beta, h} \frac{d}{d h}\left\langle\sigma_{i}\right\rangle_{\Lambda, \beta, h}\right] \\
&=\frac{\beta^{2}}{|\Lambda|} \sum_{i, j, k \in \Lambda}\left[\left\langle\sigma_{i} \sigma_{j} \sigma_{k}\right\rangle_{\Lambda, \beta, h}-\left\langle\sigma_{i}\right\rangle_{\Lambda, \beta, h}\left\langle\sigma_{j} \sigma_{k}\right\rangle_{\Lambda, \beta, h}-\left\langle\sigma_{j}\right\rangle_{\Lambda, \beta, h}\left\langle\sigma_{i} \sigma_{k}\right\rangle_{\Lambda, \beta, h}\right. \\
&\left.\quad-\left\langle\sigma_{k}\right\rangle_{\Lambda, \beta, h}\left\langle\sigma_{i} \sigma_{j}\right\rangle_{\Lambda, \beta, h}+2\left\langle\sigma_{i}\right\rangle_{\Lambda, \beta, h}\left\langle\sigma_{j}\right\rangle_{\Lambda, \beta, h}\left\langle\sigma_{k}\right\rangle_{\Lambda, \beta, h}\right] \\
& \leq 0,
\end{aligned}
$$

onde, na última desigualdade, usamos o Teorema 2.2.7 (Desigualdade GHS) para cada termo entre os colchetes acima. 
Antes de passarmos para o enunciado e prova do teorema mais importante desta seção, vamos lembrar o enunciado de um teorema clássico de Análise Convexa que será usado várias vezes adiante.

Teorema 3.4.4. Para cada $n \in \mathbb{N}$ seja $f_{n}: \mathbb{R} \rightarrow \mathbb{R}$ uma função convexa. Seja $D \subset \mathbb{R}$ um subconjunto denso. Suponha que $f_{n}(x) \rightarrow f(x)$, quando $n \rightarrow \infty$, para todo $x \in D$. Então as seguintes conclusões são válidas.

i) O limite $f(x)=\lim _{n \rightarrow \infty} f_{n}(x)$ existe para todo $x \in \mathbb{R}$ e define uma função convexa em toda reta.

ii) A sequência de funções $\left\{f_{n}\right\}$ converge uniformemente nas partes compactas da reta.

Demonstração. Veja [20] página 90.

Teorema 3.4.5. Sejam $N \in \mathbb{N}, \beta>0, h \in \mathbb{R}$ e $m_{\Lambda_{N}}(\beta, h)$ a magnetização específica do modelo de Curie-Weiss a volume $\Lambda_{N}$ com $\mathscr{J} \geq 0$. Fixado $\beta>0$, existe uma sequência de números naturais $\left\{N_{i}(\beta)\right\} \equiv\left\{N_{i}\right\}$ e $m(\beta, h) \in \mathbb{R}$, tais que para todo $h \in \mathbb{R}$ temos

$$
m(\beta, h)=\lim _{i \rightarrow \infty} m_{\Lambda_{N_{i}}}(\beta, h) .
$$

A aplicação $h \mapsto m(\beta, h)$ é côncava em $[0,+\infty)$ e contínua no aberto $(0,+\infty)$. Além do mais,

$$
-\frac{d}{d h} f(\beta, h)=m(\beta, h), \quad \forall h \in(0,+\infty) .
$$

Demonstração. Assumindo a existência do limite $\lim _{i \rightarrow \infty} m_{\Lambda_{N_{i}}}(\beta, h)$ temos que a função $h \mapsto m(\beta, h)$ é côncava pois, é limite pontual de funções côncavas. A continuidade no intervalo aberto $(0,+\infty)$ segue do fato que qualquer função real côncava definida em um aberto é uma função contínua. Portanto resta provar a existência do limite $\lim _{i \rightarrow \infty} m_{\Lambda_{N_{i}}}(\beta, h)$ sob as hipóteses do corolário.

Fixados $\beta>0$ e $\Lambda$ temos que

$$
\begin{aligned}
-\frac{d}{d h} f_{\Lambda}(\beta, h)=\frac{d}{d h}\left(\frac{1}{\beta|\Lambda|} \log Z_{\Lambda, h}\right) & =\frac{1}{\beta|\Lambda|} \frac{1}{Z_{\Lambda, h}} \frac{d}{d h} Z_{\Lambda, h} \\
& =\frac{1}{|\Lambda|}\left\langle\sum_{i \in \Lambda} \sigma_{i}\right\rangle_{\Lambda, h}=m_{\Lambda}(\beta, h) .
\end{aligned}
$$

Da igualdade acima e do Teorema Fundamental do Cálculo temos que

$$
f_{\Lambda}(\beta, 0)-f_{\Lambda}(\beta, h)=\beta \int_{0}^{h} m_{\Lambda}(\beta, x) d x
$$


Segue do Teorema 3.3 .3 (existência da pressão) que o limite do lado esquerdo da igualdade acima existe para todo $\beta>0$ e $h \in[0,+\infty)$. Vamos denotar por $f(\beta, h)$ tal limite. Lembrando que $\Lambda \equiv[-N, N] \cap \mathbb{Z}$ podemos escrever

$$
f(\beta, 0)-f(\beta, h)=\lim _{N \rightarrow \infty}\left[f_{\Lambda}(\beta, 0)-f_{\Lambda}(\beta, h)\right]=\lim _{N \rightarrow \infty} \int_{0}^{h} m_{\Lambda}(\beta, x) d x .
$$

É preciso um certo cuidado para lidar com o limite que aparece acima. Seja $\left\{q_{1}, \ldots, q_{n}, \ldots\right\}$ uma enumeração arbitrária de $\mathbb{Q}$. Como $\left|m_{\Lambda}\left(\beta, q_{1}\right)\right| \leq 1$ segue do Teorema de Bolzano-Weierstrass que existe uma sequência de números naturais $\left\{N_{i}\left(q_{1}\right)\right\}_{i \in \mathbb{N}}$ tal que a sequência $m_{\Lambda_{N_{i}\left(q_{1}\right)}}\left(\beta, q_{1}\right)$ de números reais converge para algum número real $m\left(\beta, q_{1}\right) \in[-1,1]$. Para facilitar a notação vamos escrever apenas $m_{N_{i}\left(q_{1}\right)}\left(\beta, q_{1}\right)$ para denotar $m_{\Lambda_{N_{i}\left(q_{1}\right)}}\left(\beta, q_{1}\right)$. Logo podemos escrever

$$
\lim _{i \rightarrow \infty} m_{N_{i}\left(q_{1}\right)}\left(\beta, q_{1}\right)=m\left(\beta, q_{1}\right) .
$$

Pelo mesmo argumento apresentado acima, para o racional $q_{2}$ podemos encontrar uma sequência $\left\{N_{i}\left(q_{2}\right)\right\}_{i \in \mathbb{N}}$ contida em $\left\{N_{i}\left(q_{1}\right)\right\}_{i \in \mathbb{N}}$ e um número real $m\left(\beta, q_{2}\right) \in[-1,1]$ tais que

$$
\lim _{i \rightarrow \infty} m_{N_{i}\left(q_{2}\right)}\left(\beta, q_{2}\right)=m\left(\beta, q_{2}\right) .
$$

Procedendo uma indução formal, podemos argumentar que existem sequências de números naturais $\left\{N_{i}\left(q_{j}\right)\right\}_{i \in \mathbb{N}}$ satisfazendo

$$
\left\{N_{i}\left(q_{1}\right)\right\}_{i \in \mathbb{N}} \supset\left\{N_{i}\left(q_{2}\right)\right\}_{i \in \mathbb{N}} \supset \ldots \supset\left\{N_{i}\left(q_{j}\right)\right\}_{i \in \mathbb{N}} \supset \ldots
$$

e números reais $m\left(\beta, q_{j}\right)$, para todo $j \in \mathbb{N}$, tais que

$$
\lim _{i \rightarrow \infty} m_{N_{i}\left(q_{2}\right)}\left(\beta, q_{2}\right)=m\left(\beta, q_{2}\right) .
$$

Pelas propriedades elementares de limites de sequências e pela construção acima podemos afirmar que, para qualquer $j \in \mathbb{N}$ fixado,

$$
\lim _{i \rightarrow \infty} m_{N_{i}\left(q_{i}\right)}\left(\beta, q_{j}\right)=m\left(\beta, q_{j}\right) .
$$

Isso mostra que a sequência de funções

$$
h \mapsto m_{\Lambda_{N_{i}\left(q_{i}\right)}}(\beta, h)
$$


converge em $\mathbb{Q}$. Como cada uma dessas funções é côncava e $\mathbb{Q}$ é denso em $\mathbb{R}$, podemos garantir pelo Teorema 3.4 .4 que a convergência ocorre para todo $h \in \mathbb{R}$, isto é, para todo $h \in \mathbb{R}$ temos

$$
m_{\Lambda_{N_{i}\left(q_{i}\right)}}(\beta, h) \rightarrow m(\beta, h), \quad \text { quando } i \rightarrow \infty .
$$

Como existe o limite em (3.11) segue que

$$
\lim _{N \rightarrow \infty} \int_{0}^{h} m_{\Lambda_{N}}(\beta, x) d x=\lim _{i \rightarrow \infty} \int_{0}^{h} m_{\Lambda_{N_{i}\left(q_{i}\right)}}(\beta, x) d x .
$$

Usando que $\left|m_{\Lambda_{N_{i}\left(q_{i}\right)}}(\beta, x)\right| \leq 1$ para todo $x \in \mathbb{R}$ e o Teorema da Convergência Dominada de Lebesgue, chegamos a

$$
\lim _{N \rightarrow \infty} \int_{0}^{h} m_{\Lambda_{N}}(\beta, x) d x=\lim _{i \rightarrow \infty} \int_{0}^{h} m_{\Lambda_{N_{i}\left(q_{i}\right)}}(\beta, x) d x=\int_{0}^{h} m(\beta, x) d x .
$$

Usando a igualdade acima em (3.11) ficamos com

$$
f(\beta, 0)-f(\beta, h)=\int_{0}^{h} m(\beta, x) d x .
$$

Como temos garantia que $x \rightarrow m(\beta, x)$ é contínua no intervalo $(0,+\infty)$, conclui-se da igualdade acima e do T.F.C. a diferenciabilidade de $f(\beta, h)$ em $(0,+\infty)$ e, além disso,

$$
-\frac{d}{d h} f(\beta, h)=m(\beta, h), \quad \forall h \in(0,+\infty) .
$$

No teorema acima mostramos, para todo $\beta>0$ e $h \in \mathbb{R}$, que a sequência $\left\{m_{\Lambda_{N}}(\beta, h)\right\}$ possui uma subsequência que converge para $m(\beta, h)$ e também mostramos que $m(\beta, h)$ é dada pelo negativo da derivada da energia livre. O método empregado na prova funciona para qualquer modelo para o qual podemos garantir tanto a concavidade de $x \mapsto m_{\Lambda}(\beta, x)$ como a existência da energia livre. Sua principal desvantagem é nos fornecer uma definição para $m(\beta, h)$ dependente de uma subsequência a qual não temos a priori como determinar. Apesar de não sabermos qual é a subsequência, isso não causará nenhum inconveniente no restante deste texto. Mas, de qualquer forma, é importante mencionar que no caso ferromagnético, isto é, $\mathscr{J} \geq$ 0 , é possível mostrar que a sequência $\left\{m_{\Lambda_{N}}(\beta, h)\right\}$ de fato converge para $m(\beta, h)$, assim como no modelo de Ising. 
Teorema 3.4.6. Sejam $N \in \mathbb{N}, \beta>0, h \in \mathbb{R}$ e $m_{\Lambda_{N}}(\beta, h)$ a magnetização específica do modelo de Curie-Weiss a volume $\Lambda_{N}$ com $\mathscr{J} \geq 0$. Então

$$
m(\beta, h)=\lim _{N \rightarrow \infty} m_{\Lambda_{N}}(\beta, h),
$$

onde $m(\beta, h)$ é a função obtida no teorema anterior.

Demonstração. Veja [6] Teorema IV.4.1, página 100.

\subsection{Magnetização Espontânea no Modelo de Curie-Weiss}

Vamos recapitular alguns resultados obtidos nas seções anteriores que serão usados para mostrar que a magnetização específica $m(\beta, h)$ do modelo de Curie-Weiss com constante de acoplamento $\mathscr{J} \geq 0$ satisfaz:

i) se $\beta$ é tal que $\beta \mathscr{J} \leq 1$ então $\lim _{h \rightarrow 0^{+}} m(\beta, h)=0$.

ii) se $\beta$ é tal que $\beta \mathscr{J}>1$ então $\lim _{h \rightarrow 0^{+}} m(\beta, h)>0$.

Assim, o inverso da temperatura crítica no modelo de Curie-Weiss é dado por $\beta_{c}=\mathscr{J}^{-1}$.

Pelo Teorema 3.4 .5 temos, para todo $\beta>0$ fixado, que

$$
-\frac{d}{d h} f(\beta, h)=m(\beta, h), \quad \forall h \in(0,+\infty) .
$$

Lembrando que $-f(\beta, h)=\beta^{-1} p(\beta, h)$, então podemos obter $\lim _{h \rightarrow 0^{+}} m(\beta, h)$ calculando a derivada da pressão. Mas, como temos uma fórmula explicita para a pressão $p(\beta, h)$, nosso trabalho se reduz a calcular sua derivada e, em seguida, avaliar o limite. A expressão obtida para pressão do modelo de Curie-Weiss no Teorema 3.3 .3 é a seguinte

$$
p(\beta, h)=\log (2)+\sup _{x \in \mathbb{R}}\left\{\log (\cosh (x))-\frac{(x-\beta h)^{2}}{2 \beta \mathscr{J}}\right\} .
$$

A presença do supremo na expressão acima deixa claro que para calcular a derivada de $p(\beta, h)$ com respeito a $h$ é necessário uma análise bastante cuidadosa. Vamos considerar a função auxiliar $F: \mathbb{R} \times \mathbb{R} \rightarrow \mathbb{R}$ dada por

$$
F(x, h)=\log (\cosh (x))-\frac{(x-\beta h)^{2}}{2 \beta \mathscr{J}} .
$$


Observamos que para qualquer $h \in \mathbb{R}$ fixado, temos

$$
\lim _{x \rightarrow \pm \infty} F(x, h)=-\infty .
$$

Logo, a função $x \mapsto F(x, h)$ possui pelo menos um ponto de máximo global em $\mathbb{R}$. Vamos escolher um desses pontos de máximo global e denotá-lo por $x^{*}(h)$. Como a aplicação $x \mapsto F(x, h)$ é diferenciável, a derivada dessa função calculada no ponto de máximo $x^{*}(h)$ é necessariamente nula e, portanto,

$$
0=\frac{\partial}{\partial x} F\left(x^{*}(h), h\right)=\tanh \left(x^{*}(h)\right)-\frac{\left(x^{*}(h)-\beta h\right)}{\beta \mathscr{J}} .
$$

Tomando $h=0$ na igualdade acima, ficamos com

$$
\tanh \left(x^{*}(0)\right)=\frac{x^{*}(0)}{\beta \mathscr{J}} .
$$

A partir deste ponto precisamos dividir nossa análise em três casos.

Caso-I $(\beta \mathscr{J}<1)$ Neste caso a única solução $x^{*}(0)$ para (3.13) é $x^{*}(0)=0$. De fato, a função $\tanh (x)-x /(\beta \mathscr{J})$ se anula em $x=0$ e tem derivada negativa em $\mathbb{R} \backslash\{0\}$. Considere a função $G: \mathbb{R} \times \mathbb{R} \rightarrow \mathbb{R}$ dada por

$$
G(x, h)=\tanh (x)-\frac{x-\beta h}{\beta \mathscr{J}} .
$$

Note que se $G(x, h)=0$ então $x$ é um ponto crítico de $x \mapsto F(x, h)$. Já que $G$ é de classe $C^{\infty} \mathrm{e}$

$$
\frac{\partial G}{\partial x}(0,0)=\operatorname{sech}^{2}(0)-\frac{1}{\beta \mathscr{J}}=1-\frac{1}{\beta \mathscr{J}} \neq 0
$$

segue do Teorema da Função Implícita que existe um retângulo bidimensional $R=(-\varepsilon, \varepsilon) \times(-\varepsilon, \varepsilon)$ tal que para cada $h \in(-\varepsilon, \varepsilon)$ existe um único $x_{*}(h) \in(-\varepsilon, \varepsilon)$ tal que $G\left(x_{*}(h), h\right)=0$. A aplicação $h \mapsto x_{*}(h)$ também é de classe $C^{\infty}$. Observe que para todo $|h|<\varepsilon$ então o ponto de máximo $\left(x^{*}(h), h\right)$ de $F$ satisfaz $G\left(x^{*}(h), h\right)=0$. Mas, pela unicidade fornecida pelo teorema da função implícita temos que $x^{*}(h)=x_{*}(h)$. Segue de uma observação feita acima que $h \mapsto x^{*}(h)$ define uma função de classe $C^{\infty}$. 
Se $|h|<\varepsilon$ (determinado acima) temos que

$$
\begin{aligned}
p(\beta, h) & =\log (2)+\sup _{x \in \mathbb{R}}\left\{\log (\cosh (x))-\frac{(x-\beta h)^{2}}{2 \beta \mathscr{J}}\right\} \\
& =\log (2)+\log \left(\cosh \left(x^{*}(h)\right)\right)-\frac{\left(x^{*}(h)-\beta h\right)^{2}}{2 \beta \mathscr{J}} \\
& =\log (2)+F\left(x^{*}(h), h\right) .
\end{aligned}
$$

Como todas as funções que aparecem acima são diferenciáveis com respeito a $h$, colocando $\gamma(h)=\left(x^{*}(h), h\right)$ temos pela regra da cadeia que

$$
\begin{aligned}
\frac{d}{d h} p(\beta, h) & =\frac{d}{d h} F(\gamma(h)) \\
& =\nabla F(\gamma(h)) \cdot \gamma^{\prime}(h) \\
& =\frac{\partial F}{\partial x}\left(x^{*}(h), h\right) \frac{d}{d h} x^{*}(h)+\frac{\partial F}{\partial h}\left(x^{*}(h), h\right) \\
& =\frac{\partial F}{\partial h}\left(x^{*}(h), h\right),
\end{aligned}
$$

onde na última igualdade foi usado (3.12). Usando a expressão de $F$ chegamos à seguinte igualdade

$$
\frac{d}{d h} p(\beta, h)=\frac{\partial F}{\partial h}\left(x^{*}(h), h\right)=\frac{x^{*}(h)-\beta h}{\mathscr{J}} .
$$

Do Teorema 3.4 .5 e da definição de $f(\beta, h)$ temos a seguinte igualdade $m(\beta, h)=\beta^{-1}(d / d h) p(\beta, h)$. Usando a igualdade anterior concluímos que

$$
m(\beta, h)=\frac{x^{*}(h)-\beta h}{\beta \mathscr{J}} .
$$

Mas como $x^{*}(0)=0$ e $x^{*}(h)$ é contínua próximo de zero segue

$$
\lim _{h \rightarrow 0^{+}} m(\beta, h)=0 .
$$

Caso-II $(\beta \mathscr{J}=1)$ Neste caso a única solução $x^{*}(0)$ para (3.13) é novamente $x^{*}(0)=0$. A prova desse fato é identica à do caso anterior. Agora consideramos a função $G: \mathbb{R} \times \mathbb{R} \rightarrow \mathbb{R}$ dada por

$$
G(x, h)=\tanh (x)-x+\beta h .
$$


Diferente do caso anterior, aqui não é possível aplicar imediatamente o Teorema da Função Implícita para o ponto $(0,0)$. A ideia será usar o teorema em outro ponto. Para isso observamos que existe para todo $h>0$ uma única solução positiva $x_{*}(h)$ da equação $G(x, h)=0$. De fato $G(0, h)=\beta h>0$ e, para todo $x>0$, temos $(\partial / \partial x) G(x, h)<0$. Além disso $\lim _{x \rightarrow \infty} G(x, h)=-\infty$. Como $(\partial / \partial x) G\left(x_{*}(h), h\right) \neq 0$, da unicidade garantida pelo Teorema da Função Implícita segue que $x_{*}(h)=x^{*}(h)$ para $h>0$ e que $x^{*}(h)$ é diferenciável no intervalo $(0,+\infty)$. Nesse intervalo temos

$$
\frac{d}{d h} G\left(x^{*}(h), h\right)=0 \Longrightarrow \frac{d}{d h} x^{*}(h)=\frac{\beta}{\operatorname{sech}^{2}\left(x^{*}(h)\right)-1}<0 .
$$

Já que $G\left(x^{*}(h), h\right)=0$, temos pela definição de $G$ que $\tanh \left(x^{*}(h)\right)-x^{*}(h)+\beta h=$ 0 . Usando que $|\tanh (x)| \leq 1$ segue igualdade acima $\left|x^{*}(h)\right| \leq \beta h+1$. Como $x^{*}(h)$ é também uma função decrescente de $h$ no intervalo $(0,+\infty)$ existe $\lim _{h \rightarrow 0^{+}} x^{*}(h)$. Usando a continuidade de $G$ temos que $G\left(\lim _{h \rightarrow 0^{+}} x^{*}(h), 0\right)=0$ o que implica em tanh $\left(\lim _{h \rightarrow 0^{+}} x^{*}(h)\right)=\lim _{h \rightarrow 0^{+}} x^{*}(h)$ e, portanto, $\lim _{h \rightarrow 0^{+}} x^{*}(h)=$ 0 . Como sabemos que $x^{*}(0)=0$, temos que $x^{*}$ é contínua a direita em $h=0$.

Note que a pressão, como no caso anterior, é dada por $p(\beta, h)=\log (2)+$ $F\left(x^{*}(h), h\right)$. A partir deste ponto o restante da análise pode ser feito de maneira análoga ao do caso anterior e ao final obtemos

$$
m(\beta, h)=\frac{x^{*}(h)-\beta h}{\beta \mathscr{J}} \Longrightarrow \lim _{h \rightarrow 0^{+}} m(\beta, h)=0 .
$$

Caso-III $(\beta \mathscr{J}>1)$. Para não fazer confusão com os casos anteriores, nossa escolha de um ponto de máximo global de

$$
F(x, h)=\log (\cosh (x))-\frac{(x-\beta h)^{2}}{2 \beta \mathscr{J}},
$$

(com $h>0$ fixado) será denotada por $y^{*}(0)$. Como $F$ é diferenciável sabemos que $y^{*}(0)$ deve necessariamente satisfazer a equação (3.13)

$$
\tanh \left(y^{*}(0)\right)=\frac{y^{*}(0)}{\beta \mathscr{J}} .
$$

Como estamos supondo que $\beta \mathscr{J}>1$, diferentemente do caso anterior, a equação acima não determina unicamente o ponto $y^{*}(0)$ pois, esta equação possui a solução nula e também possui uma (única) solução positiva. De fato, se $g$ denota a função dada por $g(x)=x /(\beta \mathscr{J})-\tanh (x)$ temos que $g(0)=0$ e $g^{\prime}(0)=1 /(\beta \mathscr{J})-1<0$. Como $g$ tem derivada contínua, podemos 
garantir em algum intervalo $(0, \delta)$, $\operatorname{com} \delta>0$, que $g^{\prime}$ é negativa e consequentemente $g$ é decrescente, logo negativa. Como $|\tanh (x)|<1$, temos que $\lim _{x \rightarrow+\infty} g(x)=+\infty$. Portanto existe algum ponto $y_{0} \in(0,+\infty)$ tal que $\tanh \left(y_{0}\right)=\frac{y_{0}}{\beta \not{y}}$. Já que $g^{\prime \prime}(x)=2 \tanh (x) \operatorname{sech}^{2}(x)>0$ para $x>0$, a unicidade de $y_{0}$ segue da convexidade de $g$.

Para mostrar que $y^{*}(0) \neq 0$ é suficiente mostrar que existe alguma vizinhança pequena da origem, digamos $(0, \delta)$, tal que se $y \in(0, \delta)$, então

$$
F(0, h)<F(y, h) .
$$

Daí segue que $y^{*}(0)$, o ponto de máximo global de $x \mapsto F(x, h)$ é positivo. Para isto basta observar que

$$
F(0, h)=-\frac{\beta^{2} h^{2}}{2 \beta \mathscr{J}}<F(y, h)=\log (\cosh (y))-\frac{1}{2 \beta \mathscr{J}}(y(y-\beta h x))-\frac{\beta^{2} h^{2}}{2 \beta \mathscr{J}},
$$

se $y \in(0, \beta h)$.

Procedendo exatamente como no caso anterior obtemos do Teorema da Função Implícita a existência de uma função diferenciável $y^{*}(h)$ tal que para $h>0$ temos

$$
p(\beta, h)=\log (2)+F\left(y^{*}(h), h\right) \quad \text { e } \quad m(\beta, h)=\frac{y^{*}(h)-\beta h}{\beta \mathscr{J}} .
$$

Agora tomando o limite, quando $h \rightarrow 0$ pela direita, ficamos com

$$
\lim _{h \rightarrow 0^{+}} m(\beta, h)=\frac{y^{*}(0)}{\beta \mathscr{J}}>0 .
$$

Equação Funcional da Magnetização. Em todos os três casos analisados acima, as funções $x^{*}(h)$ e $y^{*}(h)$, foram determinadas como as maiores soluções da equação

$$
\tanh (x)=\frac{(x-\beta h)}{\beta \mathscr{J}} .
$$

Para unificar a análise vamos considerar

$$
z^{*}(h)=\left\{\begin{array}{ll}
x^{*}(h), & \text { se } \beta \mathscr{J} \leq 1 \\
y^{*}(h), & \text { se } \beta \mathscr{J}>1 .
\end{array} .\right.
$$


Da afirmação feita acima temos, para todo $h \geq 0$, que a seguinte equação é satisfeita

$$
\tanh \left(z^{*}(h)\right)=\frac{\left(z^{*}(h)-\beta h\right)}{\beta \mathscr{J}} .
$$

Usando esta equação temos que

$$
\begin{aligned}
\tanh (\beta \mathscr{J} m(\beta, h)+\beta h) & =\tanh \left(\beta \mathscr{J} \frac{\left(z^{*}(h)-\beta h\right)}{\beta \mathscr{J}}+\beta h\right) \\
& =\tanh \left(z^{*}(h)\right) \\
& =\frac{\left(z^{*}(h)-\beta h\right)}{\beta \mathscr{J}} \\
& =m(\beta, h) .
\end{aligned}
$$

Para posterior referência vamos enunciar na forma de um teorema os resultados provados nesta seção. No enunciado do teorema abaixo vamos optamos por denotar a magnetização do modelo de Curie-Weiss por $m^{C W}(\beta, h)$, para diferenciá-la da magnetização dos modelos de Ising e do modelo Hierárquico, que será definido mais a frente.

Teorema 3.5.1. Seja $m^{C W}(\beta, h)$ a magnetização específica do modelo de Curie-Weiss com constante de acoplamento $\mathscr{J} \geq 0$. Então o ponto crítico desse modelo é dado por $\beta_{c}=\mathscr{J}^{-1} e$

i) se $\beta \leq \beta_{c}$, isto é, $\beta \mathscr{J} \leq 1$ então

$$
\lim _{h \rightarrow 0^{+}} m^{C W}(\beta, h)=0
$$

ii) se $\beta_{c}<\beta$, isto é, $\beta \mathscr{J}>1$ então

$$
\lim _{h \rightarrow 0^{+}} m^{C W}(\beta, h)>0
$$

iii) para todo $\beta>0$ e $h \geq 0$ temos

$$
\tanh \left(\beta \mathscr{J} m^{C W}(\beta, h)+\beta h\right)=m^{C W}(\beta, h) .
$$

\subsection{Comparação de um Modelo de Ising com Curie-Weiss}

Sejam $N \in \mathbb{N}, \Lambda=[-N, N] \cap \mathbb{Z}, \beta>0, h \geq 0$. Denote por $m_{\Lambda}(\beta, h)$ a magnetização específica no modelo de Ising no volume $\Lambda$, com condições de 
contorno livre e constantes de acoplamento $J_{i j}=J(|i-j|)$. Ao longo desta seção vamos assumir sempre que

$$
\|J\| \equiv \sum_{i=-\infty}^{\infty} J_{i j}=J(0)+2 \sum_{r=1}^{\infty} J(r)<+\infty .
$$

O objetivo desta seção é mostrar que a magnetização específica do modelo de Curie-Weiss, $m^{C W}(\beta, h)$ com $\mathscr{J}=\|J\|$ é tal que para todo $\Lambda$ vale a seguinte desigualdade

$$
\frac{1}{|\Lambda|}\left\langle\sum_{i \in \Lambda} \sigma_{i}\right\rangle_{\Lambda, \beta, h} \leq m^{C W}(\beta, h) .
$$

Lema 3.6.1. Seja $\tilde{h} \geq 0$ e considere o modelo de Ising com constantes de acoplamento $J_{i j} \equiv 0$ e hamiltoniano no volume $\Lambda$ dado por

$$
\mathscr{H}_{\Lambda}(\sigma)=-\tilde{h} \sum_{i \in \Lambda} \sigma_{i}
$$

Se $\mathbb{P}_{\Lambda, \beta}$ denota a medida de Gibbs a ao inverso da temperatura $\beta$ no volume $\Lambda$ deste modelo então, as variáveis aleatórias $\left\{X_{i}, i \in \Lambda\right\}$ definidas no espaço de probabilidade $\left(\Omega_{\Lambda}, \mathcal{F}_{\Lambda}, \mathbb{P}_{\Lambda, \beta}\right)$ por $X_{i}(\sigma)=\sigma_{i}$ são independentes.

Demonstração. Observe que a v.a. $X_{i}$ toma valores no conjunto $\{-1,1\}$ que é discreto. Portanto para provar que o coleção $\left\{X_{i}, i \in \Lambda\right\}$ é independente basta mostrar que para todo conjunto $\left\{i_{1}, \ldots, i_{k}\right\} \subset \Lambda$ e $c_{i_{1}}, \ldots, c_{i_{k}} \in\{-1,1\}$ temos

$$
\mathbb{P}_{\Lambda, \beta}\left(X_{i_{1}}=c_{1}, \ldots, X_{i_{k}}=c_{k}\right)=\prod_{j=1}^{k} \mathbb{P}_{\Lambda, \beta}\left(X_{i_{j}}=c_{j}\right) .
$$

Vamos calcular primeiro $\mathbb{P}_{\Lambda, \beta}\left(X_{i_{j}}=c_{j}\right)$. Para isto vamos começar pelo cálculo da função de partição que será denota por $\mathscr{Z}_{\Lambda, \beta}$.

$$
\begin{aligned}
\mathscr{Z}_{\Lambda, \beta}=\sum_{\sigma \in \Omega_{\Lambda}} \exp \left(-\beta \mathscr{H}_{\Lambda}(\sigma)\right) & =\sum_{\sigma_{-N} \in\{-1,1\}} \ldots \sum_{\sigma_{N} \in\{-1,1\}} \prod_{i \in \Lambda} \exp \left(\beta \tilde{h} \sigma_{i}\right) \\
& =\sum_{\sigma_{-N} \in\{-1,1\}} \ldots \sum_{\sigma_{N} \in\{-1,1\}} \prod_{i \in \Lambda} \exp \left(\beta \tilde{h} \sigma_{i}\right) \\
& =\prod_{i \in \Lambda} \sum_{\sigma_{i} \in\{-1,1\}} \exp \left(\beta \tilde{h} \sigma_{i}\right) \\
& =2^{|\Lambda|}[\cosh (\beta \tilde{h})]^{\Lambda} .
\end{aligned}
$$


Pela definição da medida de Gibbs temos que

$$
\begin{aligned}
\mathbb{P}_{\Lambda, \beta}\left(X_{i_{j}}=c_{j}\right) & =\sum_{\sigma \in \Omega_{\Lambda}} 1_{\left\{X_{i_{j}}=c_{j}\right\}} \frac{1}{\mathscr{Z}_{\Lambda, \beta}} \exp \left(-\beta \mathscr{H}_{\Lambda}(\sigma)\right) \\
& =\sum_{\sigma \in \Omega_{\Lambda}} 1_{\left\{X_{i_{j}}=c_{j}\right\}} \frac{1}{\mathscr{Z}_{\Lambda, \beta}} \prod_{i \in \Lambda} \exp \left(\beta \tilde{h} \sigma_{i}\right) \\
& =\sum_{\sigma_{-N} \in\{-1,1\}} \ldots \sum_{\sigma_{N} \in\{-1,1\}} 1_{\left\{X_{i_{j}}=c_{j}\right\}} \frac{1}{\mathscr{Z}_{\Lambda, \beta}} \prod_{i \in \Lambda} \exp \left(\beta \tilde{h} \sigma_{i}\right) \\
& =\sum_{\sigma_{-N} \in\{-1,1\}} \ldots \sum_{\sigma_{i_{j}} \in\{-1,1\}} 1_{\left\{X_{i_{j}}=c_{j}\right\}} \frac{1}{\mathscr{Z}_{\Lambda, \beta}} \prod_{i \in \Lambda} \exp \left(\beta \tilde{h} \sigma_{i}\right) \\
& =\frac{1}{2^{|\Lambda|}[\cosh (\beta \tilde{h})]^{|\Lambda|}} e^{\beta \tilde{h} c_{j}} \cdot 2^{|\Lambda|-1}[\cosh (\beta \tilde{h})]^{|\Lambda|-1} \\
& =\frac{e^{\beta \tilde{h} c_{j}}}{2 \cosh (\beta \tilde{h})} .
\end{aligned}
$$

Procedendo de maneira análoga vemos que

$$
\mathbb{P}_{\Lambda, \beta}\left(X_{i_{j}}=c_{1}, \ldots, X_{i_{k}}=c_{k}\right)=\frac{e^{\beta\left(c_{1}+\ldots c_{k}\right)}}{2^{k}[\cosh (\beta \tilde{h})]^{k}} .
$$

Desta igualdade e da expressão obtida acima para $\mathbb{P}_{\Lambda, \beta}\left(X_{i_{j}}=c_{j}\right)$ temos que (3.14) é valido e portanto o lema está demonstrado.

Corolário 3.6.2. Sejam $\left(\Omega_{\Lambda}, \mathcal{F}_{\Lambda}, \mathbb{P}_{\Lambda, \beta}\right)$ e $\left\{X_{i}, i \in \Lambda\right\}$ como no Lema (3.6.1). Denote por $\mathbb{E}_{\Lambda, \beta}$ a esperança com respeito a medida de probabilidade $\mathbb{P}_{\Lambda, \beta}$. Para todo $m \in \mathbb{R}$ fixado e toda coleção de números inteiros não-negativos $\left\{p_{i}, i \in \Lambda\right\}$ temos que

$$
\mathbb{E}_{\Lambda, \beta}\left[\prod_{i \in \Lambda}\left(m-X_{i}\right)^{p_{i}}\right]=\prod_{i \in \Lambda} \mathbb{E}_{\Lambda, \beta}\left[\left(m-X_{i}\right)^{p_{i}}\right]
$$

Demonstração. Considere a coleção de v.a.'s $\left\{Y_{i} \equiv\left(m-X_{i}\right)^{p_{i}}, i \in \Lambda\right\}$. Aplicando o Lema 3.6.1 podemos verificar que esta coleção é independente no espaço de probabilidade $\left(\Omega_{\Lambda}, \mathcal{F}_{\Lambda}, \mathbb{P}_{\Lambda, \beta}\right)$ e a igualdade afirmada acima segue das propriedades básicas de esperança de v.a.'s independentes.

Para o próximo fato que vamos provar precisamos lembrar que a medida de Gibbs $\mathbb{P}_{\Lambda, \beta}$ depende também do parâmetro $\tilde{h}$ que aparece no Hamiltoniano $\mathscr{H}_{\Lambda}$. Vamos explorar abaixo esta dependência e e para indicá-la 
vamos usar a notação $\mathbb{E}_{\Lambda, \beta, \tilde{h}}$

Lema 3.6.3. Seja $\|J\|$ como definido no início desta seção e $m^{C W}(\beta, h)$ a magnetização específica do modelo de Curie-Weiss com constante de acoplamento $\mathscr{J}=\|J\|$. Afirmamos que para todo $i \in \Lambda$

$$
m^{C W}(\beta, h)=\mathbb{E}_{\Lambda, \beta, \tilde{h}}\left[X_{i}\right], \quad \text { onde } \tilde{h}=\|J\| m^{C W}(\beta, h)+h .
$$

Demonstração. A prova deste fato segue da equação funcional obtida no item iii) do Teorema 3.5.1. Para ver isto vamos calcular o valor esperado acima com ajuda da fórmula obtida em (3.15)

$$
\begin{aligned}
\mathbb{E}_{\Lambda, \beta, \tilde{h}}\left[X_{i}\right] & =\mathbb{P}_{\Lambda, \beta, \tilde{h}}\left(X_{i}=1\right)-\mathbb{P}_{\Lambda, \beta, \tilde{h}}\left(X_{i}=-1\right) \\
& =\frac{e^{\beta \tilde{h}}}{2 \cosh (\beta \tilde{h})}-\frac{e^{-\beta \tilde{h}}}{2 \cosh (\beta \tilde{h})} \\
& =\tanh (\beta \tilde{h}) \\
& =\tanh \left(\beta\|J\| m^{C W}(\beta, h)+\beta h\right) \\
& =m^{C W}(\beta, h),
\end{aligned}
$$

onde na última igualdade usamos a equação funcional de $m^{C W}(\beta, h)$.

Lema 3.6.4. Seja $\tilde{h}=\|J\| m^{C W}(\beta, h)+h$ como no lema anterior. Para todo $i \in \Lambda$ e p inteiro não-negativo temos que

$$
\mathbb{E}_{\Lambda, \beta, \tilde{h}}\left[\left(m^{C W}(\beta, h)-X_{i}\right)^{p}\right] \geq 0 .
$$

Demonstração. Se $p$ é par não há nada a fazer. Portanto vamos assumir que $p$ é ímpar. Usando a definição do valor esperado temos que

$$
\begin{aligned}
\mathbb{E}_{\Lambda, \beta, \tilde{h}}\left[\left(m^{C W}(\beta, h)-X_{i}\right)^{p}\right] \\
=\left(m^{C W}(\beta, h)-1\right)^{p} \mathbb{P}_{\Lambda, \beta, \tilde{h}}\left(X_{i}=1\right)+\left(m^{C W}(\beta, h)+1\right)^{p} \mathbb{P}_{\Lambda, \beta, \tilde{h}}\left(X_{i}=-1\right) \\
=\frac{\left(m^{C W}(\beta, h)-1\right)^{p} e^{\beta \tilde{h}}+\left(m^{C W}(\beta, h)+1\right)^{p} e^{-\beta \tilde{h}}}{2 \cosh (\beta \tilde{h})}
\end{aligned}
$$

Aplicando o Lema 3.6 .3 temos que o lado direito da igualdade acima é dado por

$$
\frac{\left(\mathbb{E}_{\Lambda, \beta, \tilde{h}}\left[X_{i}\right]-1\right)^{p} e^{\beta \tilde{h}}+\left(\mathbb{E}_{\Lambda, \beta, \tilde{h}}\left[X_{i}\right]+1\right)^{p} e^{-\beta \tilde{h}}}{2 \cosh (\beta \tilde{h})}
$$


Usando novamente a definição de valor esperado e que $p$ é ímpar temos que

$$
\begin{aligned}
\left(\mathbb{E}_{\Lambda, \beta, \tilde{h}}\left[X_{i}\right]-1\right)^{p} & =\left(\frac{e^{\beta \tilde{h}}-e^{-\beta \tilde{h}}}{e^{\beta \tilde{h}}+e^{-\beta \tilde{h}}}-1\right)^{p} \\
& =\left(\frac{-2 e^{-\beta \tilde{h}}}{e^{\beta \tilde{h}}+e^{-\beta \tilde{h}}}\right)^{p} \\
& =\frac{-2^{p}}{2^{p}[\cosh (\beta \tilde{h})]^{p}} e^{-p \beta \tilde{h}} \\
& =\frac{-e^{-p \beta \tilde{h}}}{[\cosh (\beta \tilde{h})]^{p}}
\end{aligned}
$$

De maneira análoga temos que

$$
\begin{aligned}
\left(\mathbb{E}_{\Lambda, \beta, \tilde{h}}\left[X_{i}\right]+1\right)^{p} & =\left(\frac{e^{\beta \tilde{h}}-e^{-\beta \tilde{h}}}{e^{\beta \tilde{h}}+e^{-\beta \tilde{h}}}+1\right)^{p} \\
& =\left(\frac{2 e^{\beta \tilde{h}}}{e^{\beta \tilde{h}}+e^{-\beta \tilde{h}}}\right)^{p} \\
& =\frac{2^{p}}{2^{p}[\cosh (\beta \tilde{h})]^{p}} e^{p \beta \tilde{h}} \\
& =\frac{e^{p \beta \tilde{h}}}{[\cosh (\beta \tilde{h})]^{p}} .
\end{aligned}
$$

Usando estas duas identidades temos que

$$
\frac{\left(\mathbb{E}_{\Lambda, \beta, \tilde{h}}\left[X_{i}\right]-1\right)^{p} e^{\beta \tilde{h}}+\left(\mathbb{E}_{\Lambda, \beta, \tilde{h}}\left[X_{i}\right]+1\right)^{p} e^{\beta \tilde{h}}}{2 \cosh (\beta \tilde{h})}=\frac{-e^{-p \beta \tilde{h}} e^{\beta \tilde{h}}+e^{p \beta \tilde{h}} e^{-\beta \tilde{h}}}{2[\cosh (\beta \tilde{h})]^{p+1}} .
$$

Assim a prova do lema se reduz a mostrar que o numerador do lado direito da igualdade acima é não negativo. Já que $\tilde{h}=\|J\| m^{C W}(\beta, h)+h \geq 0$ a não negatividade do termo acima é consequência imediata da desigualdade

$$
e^{k p} e^{-k} \geq e^{-k p} e^{k}
$$

válida para todo $p$ inteiro não negativo e $k \in[0,+\infty)$.

Na sequência provamos o principal teorema desta seção. Ele mostra que a magnetização do modelo de Curie-Weiss ferromagnético com cons- 
tante de acoplamento adequadamente escolhida, majora a magnetização do modelo de Ising ferromagnetico com constantes de acoplamento invariantes por translação.

Teorema 3.6.5. Considere o modelo de Ising com Hamiltoniano a volume $\Lambda=[-N, N] \cap \mathbb{Z}$ dado por

$$
H_{\Lambda}(\sigma)=\sum_{i \in \Lambda}\left\{\sum_{j \in \Lambda}-\frac{1}{2} J_{i j} \sigma_{i} \sigma_{j}-h \sigma_{i}\right\}, \quad \operatorname{com} \quad J_{i j}=J(|i-j|), J_{k k}=0 \forall k \in \Lambda .
$$

Sejam $\|J\|_{\Lambda}=\sum_{i \in \Lambda} J_{i j}$ (está bem definido pois $J_{i j}=J(|i-j|)$ ) e $m_{\Lambda}(\beta, h)$ a magnetização específica do modelo de Ising no volume $\Lambda$. Se $m^{C W}(\beta, h)$ denota a magnetização do modelo de Curie-Weiss com constante de acoplamento $\mathscr{J}=\|J\|_{\Lambda}$, então temos para todo $\beta>0$ e $h \geq 0$ que

$$
m_{\Lambda}(\beta, h) \leq m^{C W}(\beta, h) .
$$

Demonstração. Uma manipulação algébrica simples mostra que é válida a seguinte igualdade:

$$
\begin{gathered}
-\frac{1}{2} \sum_{i, j \in \Lambda} J_{i j}\left(m^{C W}(\beta, h)-\sigma_{i}\right)\left(m^{C W}(\beta, h)-\sigma_{j}\right) \\
=-\frac{1}{2} \sum_{i \in \Lambda}\|J\|_{\Lambda}\left(m^{C W}(\beta, h)\right)^{2}+\sum_{i \in \Lambda}\|J\|_{\Lambda} m^{C W}(\beta, h) \sigma_{i} \\
-\frac{1}{2} \sum_{i, j \in \Lambda} J_{i j} \sigma_{i} \sigma_{j} .
\end{gathered}
$$

Subtraindo $h \sum_{i \in \Lambda} \sigma_{i}$ de ambos os lados da igualdade, aparece a expressão para $H_{\Lambda}(\sigma)$ no último fator do lado direito. Após uma rearrumação dos termos, chegamos à seguinte expressão:

$$
\begin{aligned}
H_{\Lambda}(\sigma)=-\frac{1}{2} \sum_{i, j \in \Lambda} J_{i j}\left(m^{C W}(\beta, h)-\sigma_{i}\right)\left(m^{C W}(\beta, h)-\sigma_{j}\right) & \\
& -\left(\|J\|_{\Lambda} m^{C W}(\beta, h)+h\right) \sum_{i \in \Lambda} \sigma_{i}+\frac{1}{2}|\Lambda|\|J\|_{\Lambda}\left(m^{C W}(\beta, h)\right)^{2} .
\end{aligned}
$$

Se agora escolhemos $\tilde{h}=\left(\|J\|_{\Lambda} m^{C W}(\beta, h)+h\right)$, é interessante definir

$$
\mathscr{H}_{\Lambda}(\sigma)=-\left(\|J\|_{\Lambda} m^{C W}(\beta, h)+h\right) \sum_{i \in \Lambda} \sigma_{i}=\tilde{h} \sum_{i \in \Lambda} \sigma_{i},
$$


para termos a seguinte igualdade

$$
\begin{array}{r}
H_{\Lambda}(\sigma)=-\frac{1}{2} \sum_{i, j \in \Lambda} J_{i j}\left(m^{C W}(\beta, h)-\sigma_{i}\right)\left(m^{C W}(\beta, h)-\sigma_{j}\right) \\
\mathscr{H}_{\Lambda}(\sigma)+\frac{1}{2}|\Lambda|\|J\|_{\Lambda}\left(m^{C W}(\beta, h)\right)^{2} .
\end{array}
$$

Denote por $\langle\cdot\rangle_{\Lambda, \beta, h}$ a esperança em $\Omega_{\Lambda}$, com respeito a medida de Gibbs definida pelo Hamiltoniano $H_{\Lambda}$ ao inverso da temperatura $\beta$. E conservando a notação dos lemas anteriores denote por $\mathbb{E}_{\Lambda, \beta, \tilde{h}}$ a esperança com respeito a medida de Gibbs definida por $\mathscr{H}_{\Lambda}$ ao inverso da temperatura $\beta$. Sejam $X_{i}: \Omega_{\Lambda} \rightarrow\{-1,1\}$ as variáveis aleatórias dadas por $X_{i}(\sigma)=\sigma_{i}$. Já que para qualquer constante (independente de $\sigma$ ) temos que a medida de Gibbs definida pelos hamiltonianos $H$ e $H+$ const. são idênticas, podemos desprezar o último termo no Hamiltoniano acima, para verificar mais facilmente que

$$
\begin{aligned}
& \left\langle m^{C W}(\beta, h)-X_{i}\right\rangle_{\Lambda, \beta, h} \\
& =\mathbb{E}_{\Lambda, \beta, h}\left[\left(m^{C W}(\beta, h)-X_{i}\right) \cdot \exp \left(\frac{1}{2} \sum_{i, j \in \Lambda} J_{i j}\left(m^{C W}(\beta, h)-X_{i}\right)\left(m^{C W}(\beta, h)-X_{j}\right)\right)\right] .
\end{aligned}
$$

Expandindo a exponencial que aparece acima em série de Taylor e usando a independência das v.a.'s $X_{i}$ estabelecida no Lema 3.6.1 e o Corolário 3.6 .2 temos que a esperança acima uma soma com coeficientes positivos de produtos da forma

$$
\mathbb{E}_{\Lambda, \beta}\left[\prod_{i \in \Lambda}\left(m^{C W}(\beta, h)-X_{i}\right)^{p_{i}}\right]=\prod_{i \in \Lambda} \mathbb{E}_{\Lambda, \beta}\left[\left(m^{C W}(\beta, h)-X_{i}\right)^{p_{i}}\right] .
$$

Aplicando agora o Lema 3.6 .4 obtemos a seguinte desigualdade

$$
\left\langle m^{C W}(\beta, h)-X_{i}\right\rangle_{\Lambda, \beta, h} \geq 0
$$

Usando a linearidade da esperança segue imediatamente que

$$
m_{\Lambda}(\beta, h) \equiv \frac{1}{|\Lambda|}\left\langle\sum_{i \in \Lambda} \sigma_{i}\right\rangle_{\Lambda, \beta, h} \equiv \frac{1}{|\Lambda|}\left\langle\sum_{i \in \Lambda} X_{i}\right\rangle_{\Lambda, \beta, h} \leq m^{C W}(\beta, h) .
$$

Teorema 3.6.6. Sob as mesmas hipóteses do teorema anterior sejam $m_{\Lambda}(\beta, h)$ e $m^{C W}\left(\beta, h,\left\|J_{\Lambda}\right\|\right)$ respectivamente as magnetizações específicas do 
modelo de Ising e Curie-Weiss ( $\left.\mathscr{J}=\left\|J_{\Lambda}\right\|\right)$ a volume $\Lambda$, então

$$
m(\beta, h)=\lim _{\Lambda \rightarrow \mathbb{Z}} m_{\Lambda}(\beta, h) \leq \lim _{\Lambda \rightarrow \mathbb{Z}} m^{C W}\left(\beta, h,\|J\|_{\Lambda}\right)=m^{C W}(\beta, h,\|J\|)
$$

onde $\|J\|=\sum_{r=1}^{\infty} J(r)$.

Demonstração. A primeira igualdade na conclusão deste teorema segue do Lema 2.4.2 a primeira desigualdade vem do teorema anterior e a última igualdade segue da seguinte igualdade

$$
m^{C W}\left(\beta, h,\|J\|_{\Lambda}\right)=\frac{z^{*}(h)-\beta}{\beta\|J\|_{\Lambda}}
$$

obtida na prova do Teorema 3.5 .1

\subsection{Acoplamento $J_{i j}=|i-j|^{-\alpha}$ \\ Casos $\alpha \leq 1 \mathbf{e} \alpha>2$}

Considere um sistema de partículas em $\Lambda \equiv[-N, N] \cap \mathbb{Z}$, cuja energia de interação entre elas é dada por $H_{\Lambda}$. Um estado fundamental deste sistema na caixa finita $\Lambda$ é um estado $\sigma \in \Omega_{\Lambda}$ que assume o menor valor possível de energia. Em outras palavras, $\sigma$ é um estado fundamental em $\Omega_{\Lambda}$ se, e somente se, $H_{\Lambda}(\sigma) \leq H_{\Lambda}(\omega)$ para todo $\omega$ em $\Omega_{\Lambda}$. Para o modelos de Ising ferromagnético em que o campo externo $h$ é constante, esses estados correspondem a todos os spins "alinhados" na direção do campo, isto é, se $h>0$, o estado fundamental é dado por $\sigma_{0}=(1,1, \ldots, 1) \in \Omega_{\Lambda}$, e caso $h<0$ o estado fundamental é dado por $\tilde{\sigma}_{0}=(-1,-1, \ldots,-1) \in \Omega_{\Lambda}$. No modelo de Ising ferromagnético é fácil ver que a inversão de qualquer dos spins do estado fundamental aumenta a energia tanto de interação com o campo externo, como de acoplamento com os demais spins. No caso simétrico $h=0$, ambos os estados com todos os spins alinhados são estados fundamentais.

Uma interação é dita uma interação somável quando a constante $M_{0}=$ $\sum_{i} J(i)$ é finita. Como a energia de interação de um spin $j$ qualquer com os demais se escreve

$$
\sigma_{j} \sum_{i \in \Lambda} J(i) \sigma_{i} \leq M_{0}
$$

A constante $2 M_{0}$ limita a energia necessária para se inverter um spin do estado fundamental se $h=0$. Mais ainda, como essa inversão tira um spin 
da menor energia de interação possível para a maior possível, $2 M_{0}$ limita a energia necessária para a inversão de qualquer spin.

Os sistemas com interação $J(i)=i^{-\alpha} \operatorname{com} \alpha \leq 1$ têm $M_{0}=\sum_{i \in \mathbb{N}^{*}} i^{-\alpha}=\infty$, logo são não-somáveis. Em [5] Dyson sugere que, nessa situação, para qualquer $\beta$ positivo fixado, a energia de interação entre os spins cresce suficientemente rápido para garantir $\lim _{N \rightarrow \infty} m_{\Lambda_{N}}^{2}=m^{2}=1$. Isso equivale a dizer que a fração de spins apontando contra a maioria vai a zero quando $N \rightarrow \infty$. Quase todos os spins se alinham na mesma direção. Por não apresentarem grande riqueza para análise, esses casos são de pouco interesse.

No outro extremo estão os modelos $\operatorname{com} \alpha>2$. Ruelle mostrou em [21] que se $M_{1}=\sum_{i} J(i) \cdot i<\infty$ não há magnetização espontânea a temperaturas finitas. Particularmente, se $J(i)=i^{-\alpha} \operatorname{com} \alpha>2$ não há transição de fase no sentido que o limite, quando $h \rightarrow 0^{+}$, da magnetização específica é nulo.

Griffiths mostrou em [13] que qualquer modelo com interações translacionalmente invariantes e somável com $M_{0}$ fixado tem magnetização não superior a um modelo de Curie-Weiss com $\mathscr{I}=M_{0}$. Demostramos esse fato neste texto quando provamos o Teorema 3.6.6. Outra prova do fato, mas para uma classe mais ampla de modelos pode ser encontrada em [18].

Como os modelos com $\alpha>1$ são somáveis, essa comparação com o modelo de Curie-Weiss implica em magnetização nula em altas temperaturas, isto é, magnetização nula sempre que $\beta \leq 1 / M_{0}$. Utilizando a estimativa costumeira de se aproximar um somatório por uma integral, temos

$$
M_{0}=\sum_{i \in \Lambda}|i|^{-\alpha} \leq 1+\int_{1}^{\infty} x^{-\alpha} d x=\frac{\alpha}{\alpha-1} .
$$

Assim, um sistema com expoente $\alpha>1$ apresenta inverso da temperatura crítica $\beta_{c} \geq 1-1 / \alpha$.

Em resumo, os resultados citados nesta seção nos permitem separar os sistemas com interação $J(i)=i^{-\alpha}$ em três classes. Se $\alpha \leq 1$ o sistema é não-somável e o módulo da magnetização é 1 em qualquer $\beta$. Se $\alpha>2 \mathrm{a}$ magnetização é nula a qualquer $\beta$.

Nesta seção não abordamos a situação $1<\alpha \leq 2$. Como esses modelos são somáveis, a comparação com Currie-Weiss implica em $\lim _{h \rightarrow 0^{+}} m(\beta, h)=$ 0 ao menos para $\beta \leq 1-1 / \alpha$. Os resultados de [5] expostos no último capítulo deste texto, nos mostram que, para $1<\alpha<2$, também há um limite inferior para $\beta$ a partir do qual a magnetização espontânea é positiva, logo a temperatura crítica é positiva e finita. 
Como já citamos, somente em 1983 foi provado em uma famoso trabalho de Frölich e Spencer [10] que para o modelo com $\alpha=2$ existe $\beta_{c}<\infty$, ou seja, há magnetização positiva a temperaturas finitas para o modelo $\operatorname{com} \alpha=2$.

\subsection{Comparando Modelos com Interações do Tipo $J(|i-j|)$}

Nossa intenção nesta seção é mostrar consequências de se identificar quais modelos com interação do tipo $J_{i j}=|i-j|^{-\alpha}$ admitem transição de fase. Veremos que essa análise nos ajuda a tirar conclusões a respeito de uma classe mais ampla de modelos de Ising.

Na situação mais simples em que $h=0$ e a interação original $J_{i j}=|i-j|^{-\alpha}$ é apenas multiplicada por uma constante, temos $\widetilde{J}_{i j}=C|i-j|^{-\alpha}$. Nesse caso a energia do sistema é também multiplicada por uma constante e

$$
\widetilde{H}_{\Lambda}(\sigma)=C \cdot H_{\Lambda}(\sigma)
$$

O fator exponencial que aparece na medida pode ser reescrito como

$$
\exp \left(-\beta \widetilde{H}_{\Lambda}(\sigma)\right)=\exp \left(-\beta C H_{\Lambda}(\sigma)\right)=\exp \left(-\widetilde{\beta} H_{\Lambda}(\sigma)\right)
$$

fazendo apenas $\widetilde{\beta}=C \beta$. Dessa forma, o modelo com energia $\widetilde{H}_{\Lambda}(\sigma)$ pode ser reescrito como um modelo com energia $H_{\Lambda}(\sigma)$, mas ao inverso da temperatura $\widetilde{\beta}=C \beta$. Ambos os sistemas são equivalentes, a menos da multiplicação da temperatura pela constante $C$. Se o modelo com energia $H_{\Lambda}$ apresentar transição de fase ao inverso da temperatura $\beta$, o sistema com energia $\widetilde{H}_{\Lambda}$ também terá uma transição de fase ao inverso da temperatura $\beta / C$.

Considere um modelo de Ising com interação translacionalmente invariante que denotaremos por $I(|i-j|)>0$. Suponha adicionalmente que $\lim _{i \rightarrow \infty} I(i)=0$. Podemos compará-lo com os modelos com interação $\widetilde{J}(|i-j|)=C|i-j|^{-\alpha}$ para descobrir se há transição de fase em alguma temperatura finita.

Se $I(|i-j|) \geq C|i-j|^{-\alpha}$ para algum $C>0$ e $\alpha<1$, então, como a magnetização quadrática não decresce com o aumento das interações $J_{i j}$, e o limite da magnetização quadrática do modelo com interações mais fracas já é 1 , necessariamente o sistema com interação $I(|i-j|)$ apresentará 
magnetização quadrática também igual a 1. Na última frase utilizamos a Desigualdade de GKS-2 ao afirmar que a magnetização quadrática não diminui com o aumento de $J_{i j}$.

No outro extremo, se $I(|i-j|) \leq C|i-j|^{-2-\epsilon}$ (para algum $0<\epsilon<1$ ), a magnetização espontânea nula para todas as temperaturas finitas no modelo com acoplamento $|i-j|^{-2-\epsilon}$ e a desigualdade GKS-2 garantem o mesmo comportamento no modelo com interações $I(|i-j|)$.

Por fim, podemos supor como verdadeiro o resultado do último capítulo onde mostramos que há magnetização positiva para modelos com interação do tipo $J(|i-j|)=C|i-j|^{-\alpha} \operatorname{com} 1<\alpha<2$ e usar esses sistemas como referência. Assim, os modelos com interação $I(|i-j|) \geq C|i-j|^{-2+\epsilon}$ apresentam magnetização positiva a partir de uma temperatura finita. Se ainda a interação $I$ é somável, também há uma região de altas temperaturas com magnetização nula. Esses sistemas têm comportamento similar ao sistema de referência com acoplamento $J(|i-j|)=|i-j|^{-2+\epsilon}$, é portanto para tais modelos existe um único $\beta_{c}$ tal que para todo $\beta<\beta_{c}$ o modelo não tem magnetização espontânea e por outro lado, se $\beta>\beta_{c}$ então existe magnetização espontânea.

Fogem a essa análise os modelos somáveis que não podem ser limitados da maneira adequada às nossas interações de referência. Também foge à comparação o caso "limite" onde $J(|i-j|)=|i-j|^{-2}$. Esse último caso foi tratado em [10].

Tabulamos abaixo os comparativos expostos nesta seção.

\begin{tabular}{|c|c|c|}
\hline Interação $I(i)$ & $\begin{array}{c}\text { Inverso da } \\
\text { Temperatura Crítica }\left(\beta_{c}\right)\end{array}$ & $\begin{array}{l}\text { Magnetização } \\
\text { Espontânea }\end{array}$ \\
\hline$I(i) \geq C i^{-1}$ & 0 & 1 \\
\hline$C_{1} i^{-2+\epsilon} \leq I(i) \leq C_{2} i^{-1-\epsilon}$ & $0<\beta_{c}<\infty$ & $\begin{cases}m\left(\beta, 0^{+}\right)=0, & \text { se } \beta<\beta_{c} \\
m\left(\beta, 0^{+}\right)>0, & \text { se } \beta>\beta_{c}\end{cases}$ \\
\hline$I(i) \leq C i^{-2-\epsilon}$ & $\infty$ & 0 \\
\hline
\end{tabular}




\section{Capítulo 4}

\section{Transição de Fase com Interações de Longo Alcance}

\subsection{Introdução}

Em 1969 era bem conhecido, como vimos na seção 1.4, que os modelos de Ising com spins interagentes tomando valores em $\{-1,+1\}$ não apresentam transição de fase se essa interação tem alcance finito. A grande novidade em modelos de Ising em uma dimensão apareceu no trabalho de Freeman Dyson [5]. Este paper traz a primeira prova de que interações de longo alcance podem originar transição de fase mesmo em sistemas do tipo Ising em uma dimensão. Além da imensa colaboração para a área com a prova da existência de transição de fase em tais modelos esse trabalho de Dyson também introduz o chamado modelo Hierárquico. Primeiramente apresentado no citado artigo para estimar a magnetização do modelo de Ising linear, o modelo Hierárquico rapidamente tornou-se um modelo de grande interesse, pelo fato de permitir uma análise bastante detalhada de suas propriedades. Várias generalizações do modelo Hierárquico de Dyson apareceram na literatura e esse modelo tem sido utilizado até os dias de hoje para atacar outros tipos de problemas.

Vimos na seção 3.7 que os modelos com interação de longo alcance com constante de acoplamento do tipo lei de potência $\left(J(i)=1 / i^{\alpha}\right)$ não apresentam transição de fase se a potência $\alpha>2$. Também vimos que os modelos $\operatorname{com} \alpha \leq 1$ são não-somáveis e apresentam magnetização 1 a qualquer temperatura. Assim, a questão mais natural é se há magnetização não-nula a temperaturas positivas no modelo de Ising quando $1<\alpha \leq 2$.

Como diminuindo qualquer das interações $J_{i j}$ não podemos aumentar 
a magnetização quadrática (ver seção 2.2), a estratégia será mostrar a existência de magnetização quadrática positiva em um sistema com interações mais fracas que o modelo de Ising original. A magnetização quadrática positiva nesse sistema com interações mais fracas implicará magnetização quadrática também positiva (maior ou igual) no sistema original para o mesmo $\beta$. Em consequência disso e do Lema 2.5.1, teremos também magnetização espontânea positiva no sistema com interações mais fortes, Na verdade este fato, magnetização quadrática positiva implica magnetização positiva, foi o resultado principal da Seção2.5.

O modelo candidato, com interações mais fracas do que a lei de potência $J(i)=1 / i^{\alpha}$, será o modelo Hierárquico apresentado na seção seguinte. A seção 4.3 comparara o modelo Hierárquico com certas constantes de acoplamento e mostrara que ele realmente tem interações mais fracas que o modelo de Ising correspondente. Em seguida, na seção 4.5 será demonstrada a existência de magnetização quadrática positiva no modelo Hierárquico para um certos conjuntos de constantes de acoplamento correspondentes a um modelo de Ising com $1<\alpha<2$. Este capítulo segue passo-a-passo o trabalho de Dyson em [5].

\subsection{O Modelo Hierárquico}

Nesta seção vamos definir o modelo Hierárquico construído em [5] e apresentar algumas de suas propriedades elementares. O modelo Hierárquico é semelhante ao modelo de Ising com exceção das constantes de acoplamento que, como no modelo de Curie-Weiss, dependem do volume. Outra diferença é que o modelo Hierárquico é definido para caixas de tamanhos específicos, para ser mais preciso o modelo Hierárquico será definido apenas para caixas de tamanho $2^{N}$, onde $N \in \mathbb{N}$.

Antes de apresentar o Hamiltoniano do modelo Hierárquico precisamos introduzir algumas definições. Vamos denotar por $\Lambda=\left\{1, \ldots 2^{N}\right\}$ uma caixa de volume $2^{N}$. Essa caixa pode ser dividida em caixas de tamanho $2^{i}, 1 \leq$ $i \leq N$. Fixada uma escala $2^{p}$, com $1 \leq p \leq N$ podemos dividir a caixa $\Lambda$ em $2^{N-p}$ caixas consecutivas de tamanho $2^{p}$, como segue

$$
\begin{aligned}
& \Lambda=\left\{1, \ldots, 2^{p}\right\} \cup\left\{2^{p}+1, \ldots, 2 \cdot 2^{p}\right\} \cup\left\{2 \cdot 2^{p}+1, \ldots, 3 \cdot 2^{p}\right\} \cup \ldots \\
& \ldots \cup\left\{(r-1) \cdot 2^{p}+1, \ldots, r \cdot 2^{p}\right\} \cup \ldots \cup\left\{\left(2^{N-P}-1\right) \cdot 2^{p}+1, \ldots, 2^{N-p} \cdot 2^{p}\right\} .
\end{aligned}
$$

Dessa forma, na escala $2^{p}$, a $r$-ésima caixa de tamanho $2^{p}$ é dada pelo se- 
guinte conjunto de sítios $\Lambda_{(p, r)}=\left\{(r-1) \cdot 2^{p}+1, \ldots, r \cdot 2^{p}\right\}$.

Para cada $\sigma \in\{-1,1\}^{\Lambda} \equiv \Omega_{\Lambda}$ denotamos por $S_{p, r}(\sigma)$ a soma dos spins em $\Lambda_{(p, r)}$, isto é,

$$
S_{p, r}(\sigma)=\sum_{i=(r-1) \cdot 2^{p}+1}^{r \cdot 2^{p}} \sigma_{i}=\sum_{i \in \Lambda_{(p, r)}} \sigma_{i} .
$$

Finalmente, para cada $N \in \mathbb{N}$ o Hamiltoniano do modelo Hierárquico no volume $\Lambda \equiv\left\{1, \ldots, 2^{N}\right\}$ é definido como sendo a função $H_{N}: \Omega_{N} \rightarrow \mathbb{R}$ dada por

$$
H_{N}(\sigma)=-\sum_{p=1}^{N} \frac{b_{p}}{2^{2 p}} \sum_{r=1}^{2^{N-p}}\left(S_{p, r}^{2}\right),
$$

onde $b_{1}, \ldots, b_{p}$ são constantes não-negativas.

Expandindo $S_{p, r}$ que aparece na expressão acima ficamos com a seguinte expressão para o Hamiltoniano

$$
H_{N}(\sigma)=-\sum_{p=1}^{N} \frac{b_{p}}{2^{2 p}} \sum_{r=1}^{2^{N-p}}\left(\sum_{i, j \in \Lambda_{(p, r)}} \sigma_{i} \sigma_{j}\right) .
$$

Assim, para determinar a contribuição do par de spins $\sigma_{i}, \sigma_{j}$ para a ener-

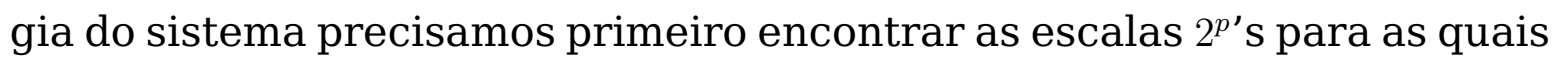
os sítios $i$ e $j$ pertençam a um mesmo bloco $\Lambda_{(p, r)}$ para algum $r$. Já que em cada uma dessas escalas $2^{p}$, esses spins contribuem com energia $b_{p} 2^{-2 p}$, temos que a contribuição total desse par de spins é obtida somando essas energias sobre tais escalas.

$\mathrm{Na}$ figura abaixo consideramos uma caixa de tamanho $2^{5}$ e uma configuração $\sigma$ fixada de $2^{5}$ spins e destacamos as escalas e o blocos que devem ser considerados na determinação da energia do par de spins $\sigma_{12}$ e $\sigma_{14}$.

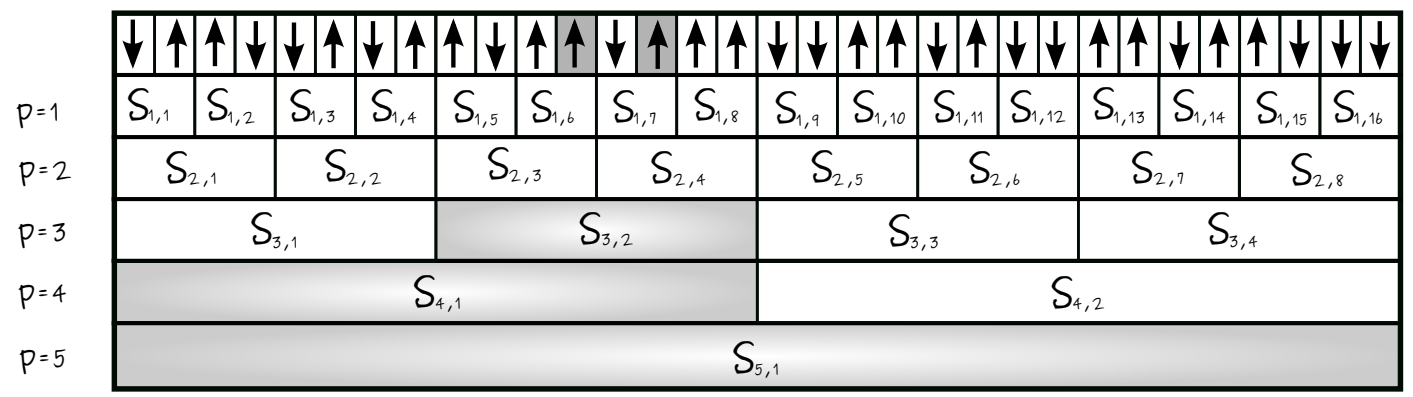

Figura 4.1: Na primeira linha, temos um exemplo de uma configuração de $2^{5}=32$ spins, onde as setas verticais representam os spins $+1 \mathrm{e}-1$. As linhas seguintes representam as caixas nas escalas 2 até $2^{5}$. Estão destacados em cinza o par de spins escolhido $\sigma_{12}$ e $\sigma_{14}$, e os blocos pelos quais eles interagem. 
No exemplo da figura acima a energia do par $\sigma_{12}$ e $\sigma_{14}$ é dada por

$$
\left(\frac{b_{3}}{2^{2 \cdot 3}}+\frac{b_{4}}{2^{2 \cdot 4}}+\frac{b_{5}}{2^{2 \cdot 5}}\right) \sigma_{12} \sigma_{14}
$$

A energia total no modelo Hierárquico é então dependente de somas como essa e, para calcular a energia total de um estado, somamos sobre todas as caixas de um certo tamanho e sobre todos os tamanhos de caixas permitidos.

O modelo Hierárquico é também um Modelo de Ising, como mencionado anteriormente, mas com constantes de acoplamento que são dependentes do volume e não são translacionalmente invariantes. Para diferenciar ambos os modelos neste capítulo, vamos nos referir ao modelo de Ising com constantes de acoplamento $J(|i-j|)=1 /|i-j|^{\alpha}$ como modelo linear.

Como foi dito acima, para calcular explicitamente a energia de interação entre dois spins específicos precisamos somar todos os termos nos quais aparece $\sigma_{i} \sigma_{j}$, ou seja, precisamos somar por todas as caixas em que os spins $i$ e $j$ estão juntos. Digamos que eles estão nas mesmas caixas $\Lambda_{(p, r)}$ para $p \geq p(i, j)$, mas em caixas diferentes se $p<p(i, j)$. Então a contribuição desse par à energia total do sistema é dada por

$$
-R_{N}(p(i, j)) \sigma_{i} \sigma_{j}=-\sum_{q=p(i, j)}^{N} \frac{b_{q}}{2^{2 q}} \sigma_{i} \sigma_{j} .
$$

Essa igualdade motiva definir uma função $R_{N}:\{1, \ldots, N\} \rightarrow \mathbb{R}$ como

$$
R_{N}(p)=\sum_{q=p}^{N} \frac{b_{q}}{2^{2 q}}
$$

$R_{N}(p(i, j))$ é a constante de acoplamento entre dois spins no modelo Hierárquico, equivalente ao que seria $J(i-j)$ no modelo linear. Contudo, no modelo Hierárquico, o equivalente à "distância" entre dois spins é o parâmetro $2^{p(i, j)}$, que é o comprimento da menor caixa a que ambos pertencem.

Outra quantidade importante é a magnetização quadrática por spin para caixas de tamanho $2^{p}$, definida por

$$
f_{N}(0) \equiv 1 \quad \text { e } \quad f_{N}(p) \equiv \frac{1}{2^{2 p}}\left\langle S_{p, r}^{2}\right\rangle
$$

Seja $\Lambda_{N+1}=\left\{1, \ldots, 2^{N+1}\right\}$. Essa caixa de tamanho $2^{N+1}$ pode ser decom- 
posta em duas caixas de tamanho $2^{N} \mathrm{e}$, usando a notação acima, podemos indicar essa decomposição da seguinte forma: $\Lambda_{N+1}=\Lambda_{(N, 1)} \cup \Lambda_{(N, 2)}$. Pela definição do modelo Hierárquico temos que $H_{N+1}$ é dado por uma soma de três parcelas, uma primeira sendo composta apenas pela energia de interação entre os spins da caixa $\Lambda_{(N, 1)}$, a segunda parcela envolve apenas interações de spins que estão na caixa $\Lambda_{(N, 2)}$ e uma terceira envolvendo spins que estão na primeira e na segunda caixa citadas acima. Essa observação é expressa pela seguinte igualdade

$$
\begin{aligned}
H_{N+1}(\sigma)=- & \sum_{i, j \in \Lambda_{(N, 1)}} R_{N+1}(p(i, j)) \sigma_{i} \sigma_{j}-\sum_{i, j \in \Lambda_{(N, 2)}} R_{N+1}(p(i, j)) \sigma_{i} \sigma_{j} \\
& -\sum_{\substack{i \in \Lambda_{(N, 1)} \\
j \in \Lambda_{(N, 2)}}} R_{N+1}(p(i, j)) \sigma_{i} \sigma_{j} .
\end{aligned}
$$

Se $i, j \in \Lambda_{(N, 1)}$ ou se $i, j \in \Lambda_{(N, 2)}$ segue da expressão obtida acima para a função $R_{N}$ que

$$
R_{N+1}(p(i, j))=\frac{b_{p(i, j)}}{2^{2 p(i, j)}}+\ldots+\frac{b_{N}}{2^{2 N}}+\frac{b_{N+1}}{2^{2 N+2}}=R_{N}(p(i, j))+\frac{b_{N+1}}{2^{2 N+2}} .
$$

Para $i \in \Lambda_{(N, 1)}$ e $j \in \Lambda_{(N, 2)}$ temos que $p(i, j)=N+1$ e assim $R_{N+1}(p(i, j))=$ $b_{2 N+1} / 2^{2 N+2}$.

Vamos olhar para as constantes $b_{1}, \ldots, b_{N+1}$ como parâmetros de $H_{N+1}$. Suponha que $\Lambda_{(p, r)} \subset \Lambda_{(N, 1)}$. Para deixar mais claro o argumento nas próximas linhas vamos usar a notação $\left\langle S_{p, r}^{2}\right\rangle_{N+1}^{b_{1}, \ldots, b_{N+1}}$ para indicar o valor esperado de $S_{p, r}^{2}$ com respeito a medida de Gibbs definida por $H_{N+1}$ no volume $\Lambda_{N+1}$ com parâmetros $b_{1}, \ldots, b_{N+1}$. Pela definição do Hamiltoniano $H_{N+1}$ e pela decomposição apresentada acima vemos que se $b_{N+1}=0$ então $H_{N+1}$ é composto por duas somas, onde os spins de uma não aparecem na outra e vice-versa. Assim é fácil ver que as v.a.'s $\sigma_{i} \operatorname{com} i \in \Lambda_{(N, 1)}$ são independentes das v.a.'s $\sigma_{j} \operatorname{com} j \in \Lambda_{(N, 2)}$, com respeito a medida de Gibbs $\langle\cdot\rangle_{N+1}^{b_{1}, \ldots, b_{N}, 0} \mathrm{e}$ daí segue que

$$
\left\langle S_{p, r}^{2}\right\rangle_{N}^{b_{1}, \ldots, b_{N}}=\left\langle S_{p, r}^{2}\right\rangle_{N+1}^{b_{1}, \ldots, b_{N}, 0}
$$

Para provar essa igualdade, precisamos estabelecer alguns fatos. Se denotamos por $\Omega_{N+1}=\{-1,1\}^{2^{N+1}}$ então $\Omega_{N+1}=\Omega_{N} \times \Omega_{N}$. Vamos denotar por $(\sigma, \omega)$ um elemento arbitrário de $\Omega_{N} \times \Omega_{N}$. Se $b_{N+1}=0$ em $H_{N+1}$ temos que a 
função de partição da medida de Gibbs determinada por $H_{N+1}$ é dada por

$$
\begin{aligned}
& Z_{N+1}^{b_{1}, \ldots, b_{N}, 0}=\int_{\Omega_{N} \times \Omega_{N}} \exp \left(H_{N+1}(\sigma, \omega)\right) d \kappa(\sigma, \omega) \\
& \quad=\int_{\Omega_{N} \times \Omega_{N}} \exp \left(\sum_{i, j \in \Lambda_{(N, 1)}} R_{N+1}(p(i, j)) \sigma_{i} \sigma_{j}+\sum_{i, j \in \Lambda_{(N, 1)}} R_{N+1}(p(i, j)) \omega_{i} \omega_{j}\right) d \kappa(\sigma, \omega) \\
& =\int_{\Omega_{N}} \exp \left(\sum_{i, j \in \Lambda_{(N, 1)}} R_{N+1}(p(i, j)) \sigma_{i} \sigma_{j}\right) d \kappa(\sigma) \int_{\Omega_{N}} \exp \left(\sum_{i, j \in \Lambda_{(N, 1)}} R_{N+1}(p(i, j)) \sigma_{i} \sigma_{j}\right) d \kappa(\sigma) \\
& =\int_{\Omega_{N}} \exp \left(\sum_{i, j \in \Lambda_{(N, 1)}} R_{N}(p(i, j)) \sigma_{i} \sigma_{j}\right) d \kappa(\sigma) \int_{\Omega_{N}} \exp \left(\sum_{i, j \in \Lambda_{(N, 1)}} R_{N}(p(i, j)) \sigma_{i} \sigma_{j}\right) d \kappa(\sigma) \\
& =Z_{N}^{b_{1}, \ldots, b_{N}} \cdot Z_{N}^{b_{1}, \ldots, b_{N}},
\end{aligned}
$$

onde na penúltima igualdade usamos a equação (4.2) juntamente com $b_{N+1}=0$. Analogamente temos que

$$
\begin{aligned}
\left\langle S_{p, r}^{2}\right\rangle_{N+1}^{b_{1}, \ldots, b_{N}, 0} & =\frac{1}{Z_{N+1}^{b_{1}, \ldots, b_{N}, 0}} \int_{\Omega_{N} \times \Omega_{N}} S_{p, r}^{2}(\sigma, \omega) \exp \left(H_{N+1}(\sigma, \omega)\right) d \kappa(\sigma, \omega) . \\
& =\frac{1}{Z_{N}^{b_{1}, \ldots, b_{N}} \cdot Z_{N}^{b_{1}, \ldots, b_{N}}} \int_{\Omega_{N} \times \Omega_{N}} S_{p, r}^{2}(\sigma) \exp \left(H_{N+1}(\sigma, \omega)\right) d \kappa(\sigma, \omega) . \\
& =\frac{Z_{N}^{b_{1}, \ldots, b_{N}}}{Z_{N}^{b_{1}, \ldots, b_{N}} \cdot Z_{N}^{b_{1}, \ldots, b_{N}}} \int_{\Omega_{N}} S_{p, r}^{2}(\sigma) \exp \left(H_{N}(\sigma)\right) d \kappa(\sigma) \\
& =\frac{1}{Z_{N}^{b_{1}, \ldots, b_{N}}} \int_{\Omega_{N}} S_{p, r}^{2}(\sigma) \exp \left(H_{N}(\sigma)\right) d \kappa(\sigma) \\
& =\left\langle S_{p, r}^{2}\right\rangle_{N}^{b_{1}, \ldots, b_{N}} .
\end{aligned}
$$

O próximo passo é mostrar que se $b_{N+1} \geq 0$ então temos

$$
\left\langle S_{p, r}^{2}\right\rangle_{N+1}^{b_{1}, \ldots, b_{N}, 0} \leq\left\langle S_{p, r}^{2}\right\rangle_{N+1}^{b_{1}, \ldots, b_{N}, b_{N+1}} .
$$

Para ver que isso é verdadeiro, basta lembrar que

$$
S_{p, r}^{2}=\sum_{i, j \in \Lambda_{(p, r)}} \sigma_{i} \sigma_{j}
$$


e que podemos aplicar GKS-2 para garantir

$$
\frac{\partial}{\partial b_{N+1}}\left\langle S_{p, r}^{2}\right\rangle_{N+1}^{b_{1}, \ldots, b_{N}, b_{N+1}} \geq 0 .
$$

Voltando a omitir os parâmetros $b_{i}$ 's da notação, o que acabamos de provar é que $\left\langle S_{p, r}^{2}\right\rangle_{N} \leq\left\langle S_{p, r}^{2}\right\rangle_{N+1}$. Dividindo ambos os lados dessa desigualdade por $2^{2 p}$, temos finalmente que $f_{N}(p) \leq f_{N+1}(p)$. Como a sequência $f_{N}(p)$ está contida no intervalo $[-1,+1]$, podemos afirmar, para cada $p \in \mathbb{N}$ fixado, que existe o limite

$$
f(p)=\lim _{N \rightarrow \infty} f_{N}(p)
$$

Por simetria do Hamiltoniano (4.1), a quantidade $\left\langle S_{p, r}^{2}\right\rangle_{N}$ é independente da caixa específica $r$ e depende apenas da escala $2^{p}$. Podemos usar essa simetria para desenvolver a expressão de $f_{N}(p+1)$. Como uma caixa na escala $2^{p+1}$ contém duas caixas na escala $2^{p}$, podemos escrever

$$
S_{p+1, r}=S_{p, 2 r-1}+S_{p, 2 r} .
$$

Usando essa relação na expressão de $f_{N}(p+1)$ podemos obtemos a seguinte igualdade

$$
\begin{aligned}
f_{N}(p+1) & =2^{-2 p-2}\left\langle S_{p+1, r}^{2}\right\rangle \\
& =2^{-2 p-2}\left[\left\langle S_{p, 2 r-1}^{2}\right\rangle+\left\langle S_{p, 2 r}^{2}\right\rangle+2\left\langle S_{p, 2 r-1} S_{p, 2 r}\right\rangle\right] \\
& =2^{-2 p-2}\left[2\left\langle S_{p, 1}^{2}\right\rangle+2\left\langle S_{p, 2 r-1} S_{p, 2 r}\right\rangle\right] .
\end{aligned}
$$

Novamente por simetria, podemos substituir as caixas $(p, 2 r-1)$ e $(p, 2 r)$ por $(p, 1)$ e $(p, 2)$, respectivamente. Assumindo que $\left\langle S_{p, 1} S_{p, 2}\right\rangle \leq\left\langle S_{p, 1}^{2}\right\rangle$ poderíamos então concluir que $f_{N}(p+1) \leq f_{N}(p)$. Apesar da função ser de fato não-crescente em $p$ e, consequentemente, existir o $\operatorname{limite}_{\lim _{p \rightarrow \infty}} f(p)$, a demonstração desse resultado é desnecessária para nossa análise e a omitiremos.

Na Seção 4.5 serão feitas estimativas diretamente para a quantidade $f_{N}(N)=2^{-2 N}\left\langle S_{N, 1}^{2}\right\rangle$, que equivale exatamente à magnetização quadrática por spin. Por isso, estaremos interessados em mostrar que

$$
m_{H}^{(2)}=\liminf _{N \rightarrow \infty} \frac{1}{2^{2 N}}\left\langle S_{N, 1}^{2}\right\rangle=\liminf _{N \rightarrow \infty} f_{N}(N)
$$

é positivo. 
A monotonicidade de $f(p)$ fornece um resultado adicional:

$$
m_{H}^{(2)}=\liminf _{N \rightarrow \infty} f_{N}(N) \leq \lim _{p \rightarrow \infty} f(p) .
$$

Logo, a positividade de $m_{H}^{(2)}$ acarreta em $\lim _{p \rightarrow \infty} f(p)$ também não-nulo.

\subsection{Comparando os Modelos Hierárquico e de Ising}

O objetivo desta seção é encontrar um modelo Hierárquico, determinado pelas constantes $b_{p}$, com interações mais fracas que um dado modelo de Ising com interações $J_{i j} \equiv J(|i-j|) \geq 0$. Para a comparação se tornar mais simples a ideia é definir um modelo de Ising na rede $\left\{1, \ldots, 2^{N}\right\}$, usando as constantes de acoplamento $J_{i j}$. Em seguida, escolhemos constantes $b_{p}$ 's e definimos um modelo Hierárquico na rede $\left\{1, \ldots, 2^{N}\right\}$, de forma que este modelo Hierárquico tenha interação mais fraca que a interação do modelo de Ising. Construído tal modelo Hierárquico, podemos concluir da Desigualdade GKS-2 que a magnetização deste modelo é menor ou igual a magnetização do modelo de Ising na rede $\left\{1, \ldots, 2^{N}\right\}$ com constantes de acoplamento dadas por $J_{i j}$. Estabelecida a desigualdade mencionada acima precisamos a rigor ainda cuidar de uma questão técnica. Se $\Gamma_{N}=\left\{1, \ldots, 2^{N}\right\}$ e $m_{\Gamma}(\beta, h)$ denota a magnetização específica do modelo de Ising mencionado acima, com constantes de acoplamento $J_{i j}$ precisamos mostrar que

$$
m_{\Gamma}(\beta, h) \rightarrow m(\beta, h),
$$

onde $m(\beta, h)$ é a magnetização específica do modelo de Ising que foi definida anteriormente como $\lim _{N \rightarrow \infty} m_{\Lambda_{N}}(\beta, h)$, onde $\Lambda_{N}=[-N, N] \cap \mathbb{Z}$.

Para provar a afirmação feita acima, sobre a igualdade dos limites termodinâmicos basta lembrar que provamos na Seção 2.4, que se as constantes de acoplamento dependem apenas da distância entre os sítios, isto é, $J_{i j}=J(|i-j|)$ e são não-negativas, então existe o $\operatorname{limite}_{\lim _{N \rightarrow \infty}} m_{\Delta_{N}}(\beta, h)$, onde $\Delta_{N}=\{1, \ldots, N\}$. Este limite é igual a $m(\beta, h)$. Obviamente $\left\{m_{\Gamma}(\beta, h)\right\}$ é uma subsequência de $\left\{m_{\Delta_{N}}(\beta, h)\right\}$ e portanto também converge para $m(\beta, h)$. Também argumentamos na Seção 2.4 que $\lim _{N \rightarrow \infty} m_{\Lambda_{N}}(\beta, h)=m(\beta, h)$ e portanto a afirmação está provada.

Em [5] Dyson escolhe as seguintes constantes $b_{p}$ para compará-las, em 
seguida, com $\mathbf{J}$ :

$$
b_{p}=\min _{1 \leq q \leq p}\left[2^{p+q-2} J\left(2^{q}-1\right)\right] .
$$

No modelo Hierárquico com essas constantes, a contribuição à energia total dada por dois spins $\sigma_{j}$ e $\sigma_{k}$, com $j, k \in\left\{1, \ldots, 2^{N}\right\}$ é exatamente $R_{N}(p(j, k))$. Pela definição de $p(j, k)$ temos que a distância entre $j$ e $k$ na rede pode ser estimada por $|j-k| \leq 2^{p(j, k)}-1$, assim segue direto da definição de $b_{p}$ que

$$
\begin{aligned}
R_{N}(p(j, k))=\sum_{q=p(j, k)}^{N} 2^{1-2 q} b_{q} & =\sum_{q=p(j, k)}^{N} 2^{1-2 q} \min _{1 \leq r \leq q}\left[2^{q+r-2} J\left(2^{r}-1\right)\right] \\
& \leq \sum_{q=p(j, k)}^{N} 2^{p(j, k)-q-1} J\left(2^{p}-1\right) \\
& \leq J(|j-k|)
\end{aligned}
$$

A Desigualdade (4.5) juntamente com o Corolário 2.2.5nos mostra que, dado um modelo de Ising com constantes de acoplamento $J(|j-k|)$, se há magnetização não-nula para $\beta>\beta_{0}$ no modelo Hierárquico correspondente (com os $b_{p}$ 's construído acima), também haverá magnetização positiva no modelo linear para todo $\beta>\beta_{0}$.

Observamos que o modelo Hierárquico com $b_{p}=2^{(2-\alpha) p}$ tem interações mais fracas que o modelo linear com $J(i)=1 / i^{\alpha}$. De fato, para todos os sítios $j$ e $k$ da rede tais que $|j-k| \leq 2^{p}-1$, onde $p \equiv p(j, k)$ temos

$$
R_{N}(p)=\sum_{q=p}^{N} 2^{1-2 q} b_{q}=\sum_{q=p}^{N} 2^{1-2 q} 2^{(2-\alpha) q} \leq \sum_{q=p}^{N} 2 \frac{1}{2^{\alpha p}} \leq \frac{1}{|j-k|^{\alpha}}
$$

\subsection{Magnetização Quadrática e Magnetização Espontânea}

Já havíamos mencionado que o objetivo deste capítulo é demonstrar a existência de magnetização quadrática não-nula para uma classe de modelos Hierárquicos. Para permitir um aproveitamento ótimo das simetrias desses modelos, eles foram definidos sem a presença de campo externo, ou seja, $h_{i}=0$. Será necessário, então, conectar a presença de magnetização quadrática não-nula em um modelo Hierárquico com constantes $b_{p}=2^{(2-\alpha) p}$ com a magnetização espontânea num modelo linear com cons- 
tantes de acoplamento $J(i)=i^{-\alpha}$.

Já sabemos da seção anterior que as constantes de acoplamento do modelo linear com expoente $\alpha$ superam as do modelo Hierárquico com constante de acoplamento correspondentes, dadas por $b_{p}=2^{(2-\alpha) p}$. Adiante nesta seção a Desigualdade GKS-2 nos garantirá que o aumento nas constantes de acoplamento $J_{i j}$ não reduz a magnetização quadrática, assim teremos

$$
m_{L, \Lambda_{N}, 0}^{(2)} \geq m_{H, \Lambda_{N}, 0}^{(2)} .
$$

Onde $m_{L, \Lambda_{N}, 0}^{(2)}$ representa a magnetização quadrática no modelo linear (índice $L$ ), na caixa $\Lambda_{N}$ e com campo externo $h=0$.

Também veremos que o aumento no campo externo não diminui a magnetização quadrática, ou seja,

$$
m_{L, \Lambda_{N}, h}^{(2)} \geq m_{L, \Lambda_{N}, 0}^{(2)} .
$$

Concatenando as duas últimas inequações e tomando seus limites inferiores, chegamos a

$$
\liminf _{N \rightarrow \infty} m_{L, \Lambda_{N}, h}^{(2)} \geq \liminf _{N \rightarrow \infty} m_{H, \Lambda_{N}, 0}^{(2)}=m_{H}^{(2)}>0 .
$$

Podemos, então, utilizar os Lemas 2.4.2 e 2.5.1 para concluir

$$
\liminf _{N \rightarrow \infty} m_{L, \Lambda_{N}, h}^{(2)} \geq m_{H}^{(2)}>0 \Rightarrow \lim _{N \rightarrow \infty} m_{L, \Lambda_{N}, h}=m_{L, h} \geq m_{H}^{(2)}>0 .
$$

A desigualdade acima mostra que a magnetização no modelo linear para todo campo externo $h>0$ é uniformemente limitada pela constante $m_{H}^{(2)}$, logo o limite de $m(\beta, h)$ quando $h \rightarrow 0^{+}$também é limitado por essa constante. Assim, a magnetização espontânea no modelo linear é maior ou igual à magnetização quadrática no modelo Hierárquico (com campo externo $h=0$ ). Na seção seguinte serão apresentadas condições suficientes para termos

$$
\liminf _{N \rightarrow \infty} m_{H, \Lambda_{N}, 0}^{(2)}=m_{H}^{(2)}>0
$$

e, consequentemente, magnetização espontânea positiva no modelo linear correspondente.

Sigamos com a demonstração das desigualdades (4.7) e (4.8). Os métodos utilizados serão os mesmos da demonstração do 2.2.5. Vamos começar provando (4.8). A ideia é calcular $\partial m_{\Lambda_{N}}^{(2)} / \partial h$ e mostrar que essa derivada é 
sempre não-negativa para quando $h \geq 0$.

$$
\begin{aligned}
\frac{\partial}{\partial h} m_{\Lambda}^{(2)} & =\frac{\partial}{\partial h} \int_{\Omega_{\Lambda}}\left(\frac{\sum_{i \in \Lambda} \sigma_{i}}{|\Lambda|}\right)^{2} \mathbb{P}_{\Lambda}(\sigma) d \kappa(\sigma) \\
& =|\Lambda|^{-2} \int_{\Omega_{\Lambda}} \sum_{i, j \in \Lambda} \sigma_{i} \sigma_{j} \frac{\partial}{\partial h} \mathbb{P}_{\Lambda}(\sigma) d \kappa(\sigma) .
\end{aligned}
$$

Trazendo o resultado de $\partial \mathbb{P}_{\Lambda}(\sigma) / \partial h$ calculada no ponto $\sigma$ da Seção 2.2, chegamos à expressão

$$
\begin{aligned}
\frac{\partial}{\partial h} m_{\Lambda}^{(2)} & =\beta|\Lambda|^{-2} \int_{\Omega_{\Lambda}} \sum_{i, j \in \Lambda} \sigma_{i} \sigma_{j}\left[\sum_{k \in \Lambda} \sigma_{k}-\left\langle\sum_{k \in \Lambda} \sigma_{k}\right\rangle_{\Lambda}\right] \mathbb{P}_{\Lambda}(\sigma) d \kappa(\sigma) \\
& =\beta|\Lambda|^{-2} \sum_{i, j, k \in \Lambda}\left[\left\langle\sigma_{i} \sigma_{j} \sigma_{k}\right\rangle_{\Lambda}-\left\langle\sigma_{i} \sigma_{j}\right\rangle_{\Lambda}\left\langle\sigma_{k}\right\rangle_{\Lambda}\right] \geq 0 .
\end{aligned}
$$

A última desigualdade acima segue da aplicação da Desigualdade GKS-2 a cada parcela do somatório. Fica assim demonstrada a desigualdade (4.8).

Repetindo o mesmo procedimento, podemos confirmar a desigualdade (4.7). Neste caso, derivaremos $\partial m_{\Lambda_{N}}^{(2)} / \partial J_{i j}$.

$$
\begin{aligned}
\frac{\partial}{\partial J_{i j}} m_{\Lambda}^{(2)} & =|\Lambda|^{-2} \int_{\Omega_{\Lambda}} \sum_{k, l \in \Lambda} \sigma_{k} \sigma_{l} \frac{\partial}{J_{i j}} \mathbb{P}_{\Lambda}(\sigma) \pi_{\Lambda}(d \sigma) . \\
& =\beta|\Lambda|^{-2} \int_{\Omega_{\Lambda}} \sum_{k, l \in \Lambda} \sigma_{k} \sigma_{l}\left[\sigma_{i} \sigma_{j}-\left\langle\sigma_{i} \sigma_{j}\right\rangle_{\Lambda}\right] \mathbb{P}_{\Lambda}(\sigma) \pi_{\Lambda}(d \sigma) \\
& =\beta|\Lambda|^{-2} \sum_{k, l \in \Lambda}\left[\left\langle\sigma_{i} \sigma_{j} \sigma_{k} \sigma_{l}\right\rangle_{\Lambda}-\left\langle\sigma_{i} \sigma_{j}\right\rangle_{\Lambda}\left\langle\sigma_{k} \sigma_{l}\right\rangle_{\Lambda}\right] \geq 0
\end{aligned}
$$

A conclusão desta seção é que demonstrar a positividade da magnetização quadrática em um sistema Hierárquico (sem campo externo) implica na existência de magnetização espontânea positiva em qualquer outro sistema com acoplamento mais forte. Em particular, a existência de magnetização quadrática positiva em um sistema Hierárquico com contantes $b_{p}=2^{(2-\alpha) p}$ implica na magnetização espontânea positiva num modelo linear com constantes de acoplamento $J(i)=i^{-\alpha}$, estando ambos os sistemas no mesmo inverso da temperatura $\beta$. 


\subsection{Magnetização Quadrática Não-nula em Baixas Temperaturas}

Nesta seção, nossa intenção é seguir a prova da magnetização quadrática não-nula no modelo Hierárquico dado um certo conjunto de constantes $b_{p}$ da forma $b_{p}=2^{(2-\alpha) p} \operatorname{com} 1<\alpha<2$.

Por magnetização quadrática, nos referimos a:

$$
m_{\Lambda}^{(2)}=\left\langle s_{\Lambda}^{2}\right\rangle_{\Lambda}=\left\langle\left[\frac{S}{|\Lambda|}\right]^{2}\right\rangle_{\Lambda}=\left\langle\left[\frac{\sum_{i \in \Lambda} \sigma_{i}}{|\Lambda|}\right]^{2}\right\rangle_{\Lambda}=\frac{\left\langle\left(\sum_{i \in \Lambda} \sigma_{i}\right)^{2}\right\rangle_{\Lambda}}{|\Lambda|^{2}},
$$

onde os valor esperado acima é tomado com respeito a medida de Gibbs definida pelo Hamiltoniano $H_{N}$ do modelo Hierárquico no volume $\Lambda=$ $\left\{1, \ldots, 2^{N}\right\}$.

Começamos com o seguinte lema que será usado durante a prova do teorema principal.

Lema 4.5.1. Seja $\left\{x_{1}, x_{2}, \ldots, x_{n}\right\}$ um conjunto de números inteiros, tais que $\forall i \in\{1,2, \ldots, n\}$ temos $0 \leq x_{i} \leq a$. Seja $f: \mathbb{R} \rightarrow \mathbb{R}$ uma função real positiva e $b$ um número real, então:

$$
\sum_{i} \sum_{j} \exp \left[-\frac{\left(x_{i}-x_{j}\right)^{2}}{b^{2}}\right] f\left(x_{i}\right) f\left(x_{j}\right) \geq \frac{e^{-1} b}{a+b}\left(\sum_{i} f\left(x_{i}\right)\right)^{2}
$$

Demonstração. Seja $q$ número inteiro dado por $q=\lceil a / b\rceil+1$, onde $\lceil a / b\rceil$ é o menor inteiro maior que $a / b$. Vamos dividir o intervalo $[0, a]$ em $q$ subintervalos da forma $I_{p}=[p / q,(p+1) / q]$, $\operatorname{com} p=1, \ldots, q$.

A principal ideia para obter a estimativa que estamos interessados é descartar parcelas da forma $\exp \left[-\left(x_{i}-x_{j}\right)^{2} / b\right]$, onde os pontos $x_{i}$ e $x_{j}$ estão relativamente distantes com respeito a escala dada pelo comprimento do intervalo $I_{k}$. Assim, vamos manter apenas as parcelas cujos pares $\left\{x_{i}, x_{j}\right\}$ estejam contidos no mesmo intervalo $I_{k}$.

Denote por $F_{k}$ a seguinte soma

$$
F_{k}=\sum_{x_{i} \in I_{k}} f\left(x_{i}\right)
$$


Pela definição de $I_{k}$ se $x_{i}, x_{j} \in I_{k}$ temos que $\left|x_{i}-x_{j}\right| \leq a / q$ e portanto

$$
\begin{aligned}
\sum_{i} \sum_{j} \exp \left[-\frac{\left(x_{i}-x_{j}\right)^{2}}{b^{2}}\right] f\left(x_{i}\right) f\left(x_{j}\right) & \geq \exp \left[-\frac{1}{b^{2}}\left(\frac{a}{q}\right)^{2}\right] \sum_{k} \sum_{x_{i}, x_{j} \in I_{k}} f\left(x_{i}\right) f\left(x_{j}\right) \\
& =\exp \left[-\frac{1}{b^{2}}\left(\frac{a}{q}\right)^{2}\right] \sum_{k} F_{k}^{2} .
\end{aligned}
$$

Usando que $a / b<q$, obtemos a seguinte cota inferior para o lado direito da desigualdade acima

$$
\exp \left[\left(-\frac{a}{b} \cdot \frac{1}{q}\right)^{2}\right] \sum_{k} F_{k}^{2}>\exp (-1) \sum_{k} F_{k}^{2} .
$$

Visto que a média quadrática é maior que a média aritmética, podemos deduzir que

$$
\sqrt{\sum_{k=1}^{q} \frac{F_{k}^{2}}{q}} \geq \sum_{k=1}^{q} \frac{F_{k}}{q} \Longrightarrow \sqrt{q \cdot \sum_{k=1}^{q} F_{k}^{2}} \geq \sum_{k} F_{k} \Longleftrightarrow \sum_{k=1}^{q} F_{k}^{2} \geq \frac{1}{q}\left(\sum_{k} F_{k}\right)^{2} .
$$

Aplicando a última desigualdade acima em (4.9) obtemos

$$
\begin{aligned}
\sum_{i} \sum_{j} \exp \left[-\frac{\left(x_{i}-x_{j}\right)^{2}}{b^{2}}\right] f\left(x_{i}\right) f\left(x_{j}\right) & \geq e^{-1} \sum_{k} F_{k}^{2} \\
& \geq \frac{1}{e \cdot q}\left(\sum_{k} F_{k}\right)^{2} \\
& \geq \frac{1}{e \cdot q}\left(\sum_{i} f\left(x_{i}\right)\right)^{2}
\end{aligned}
$$

Já que $q \leq a / b+1=(a+b) / b$ concluímos finalmente que

$$
\sum_{i} \sum_{j} \exp \left[-\frac{\left(x_{i}-x_{j}\right)^{2}}{b^{2}}\right] f\left(x_{i}\right) f\left(x_{j}\right) \geq \frac{b \cdot e^{-1}}{a+b}\left(\sum_{x_{i} \in I_{k}} f\left(x_{i}\right)\right)^{2}
$$

o que prova o Lema.

Com esse lema em mãos podemos voltar ao tema principal, o teorema mais importante desta seção, cujo o enunciado é o seguinte.

Teorema 4.5.2. Sejam $\left\{b_{p}\right\}_{p \in \mathbb{N}}$ as constantes usadas para definir o Hamiltoniano do modelo Hierárquico na Seção 4.2. Se convergir a seguinte 
quantidade:

$$
L=\sum_{p=1}^{\infty} \frac{1}{b_{p}} \log (1+p)
$$

então haverá magnetização quadrática positiva ao inverso da temperatura $\beta$ desde que $\beta>8 L$.

Demonstração. Inicialmente, escrevemos a função de partição do modelo Hierárquico como segue

$$
Z_{N}=\sum_{s} Z_{N}(s)
$$

onde $Z_{N}(s)$ é a soma de todas as parcelas da função de partição tal que $\sigma_{1}+\ldots+\sigma_{2^{N}}=s$. Podemos pensar nas $Z_{N}(s)$ como funções de partição correspondente aos estados com magnetização total $s$.

Para cada $N \in \mathbb{N}$ seja $\zeta_{N}: \mathbb{R} \rightarrow \mathbb{R}$ a função dada por

$$
\zeta_{N}(u)=\sum_{s} \exp \left(u s^{2}\right) Z_{N}(s)
$$

As funções $\zeta_{N}$ definidas dessa maneira gozam da seguinte propriedade $\zeta_{N}(0)=\sum_{s} Z_{N}(s)=Z_{N}$. Definimos também a seguinte função

$$
L_{N}(u)=\log \left(\zeta_{N}(u)\right)
$$

Afirmamos que $L_{N}$ é uma função convexa com respeito à variável $u$. De fato, basta calcular $L_{N}\left(\lambda u_{1}+(1-\lambda) u_{2}\right)$ e mostrar, usando a Desigualdade de Hölder que $L_{N}\left(\lambda u_{1}+(1-\lambda) u_{2}\right) \leq \lambda L_{N}\left(u_{1}\right)+(1-\lambda) L\left(u_{2}\right)$.

Um cálculo direto mostra que a derivada de $L_{N}$ é dada por

$$
L_{N}^{\prime}(u)=\left[\log \left(\zeta_{N}(u)\right)\right]^{\prime}=\frac{\zeta_{N}(u)^{\prime}}{\zeta_{N}(u)}=\frac{\sum_{s} s^{2} \exp \left(u s^{2}\right) Z_{N}(s)}{\sum_{s} \exp \left(u s^{2}\right) Z_{N}(s)}
$$

e que essa igualdade cria uma conexão entre $L_{N}$ e a esperança da magnetização quadrática, uma vez que $L_{N}^{\prime}(0)=\left\langle S_{2^{N}}^{2}\right\rangle_{N}=2^{2 N} f_{N}(N)$.

Levando em conta a estrutura hierárquica das somas (4.3) podemos decompor o espaço de configurações $\Omega_{\Lambda_{2^{N}}}$ através dos valores das magnetizações totais em $\Lambda_{\left(2^{N-1}, 1\right)}$ e $\Lambda_{\left(2^{N-1}, 2\right)}$, que denotaremos por $x$ e $y$ respectivamente. Assim, para cada configuração $\sigma \in \Omega_{\Lambda_{2^{N}}}$ cujas as magnetizações totais em $\Lambda_{\left(2^{N-1}, 1\right)}$ e $\Lambda_{\left(2^{N-1}, 2\right)}$ são dadas respectivamente por $x$ e $y$ temos que 
a energia de interação entre essas duas caixas é dada por

$$
H_{\text {out }}(x, y)=2^{-2 N} b_{N}\left(S_{N, 1}\right)^{2}=2^{-2 N} b_{N}\left(S_{N-1,1}+S_{N-1,2}\right)^{2}=2^{-2 N} b_{N}(x+y)^{2}
$$

Já que todos os valores possíveis de magnetizações totais em $\Lambda_{\left(2^{N-1}, 1\right)}$ ou $\Lambda_{\left(2^{N-1}, 2\right)}$ pertencem ao conjunto $A=\left\{-2^{N-1},-2^{N-1}+2, \ldots, 2^{N-1}-2,2^{N-1}\right\}$, podemos escrever a função de partição do sistema inteiro como segue

$$
Z_{N}=\sum_{x \in A} \sum_{y \in A} Z_{N-1}(x) Z_{N-1}(y) e^{-\beta H_{\text {out }}(x, y)} .
$$

Usando a igualdade acima, obtemos a seguinte representação de $\zeta_{N}$

$$
\begin{aligned}
\zeta_{N}(u) & =\sum_{s} \exp \left(u s^{2}\right) Z_{N}(s)=\sum_{x \in A} \sum_{y \in A} \exp \left(u(x+y)^{2}\right) Z_{N-1}(x) Z_{N-1}(y) e^{\beta H_{\text {out }}(x, y)} \\
& =\sum_{x \in A} \sum_{y \in A} \exp \left(u(x+y)^{2}\right) Z_{N-1}(x) Z_{N-1}(y) \exp \left(\beta 2^{-2 N} b_{N}(x+y)^{2}\right) \\
& =\sum_{x \in A} \sum_{y \in A} \exp \left(\left(u+\beta 2^{-2 N} b_{N}\right) \cdot(x+y)^{2}\right) Z_{N-1}(x) Z_{N-1}(y) \\
& =\sum_{x \in A} \sum_{y \in A} \exp \left((u+v) \cdot(x+y)^{2}\right) Z_{N-1}(x) Z_{N-1}(y)
\end{aligned}
$$

onde $v$ é definido como $v=\beta 2^{-2 N} b_{N}$.

Será necessário estimar $\zeta_{N}$ para alguns valores de $u$. Vamos considerar primeiro $u=-v$ :

$$
\zeta_{N}(-v)=\sum_{x \in A} \sum_{y \in A} \exp (0) Z_{N-1}(x) Z_{N-1}(y)=\left[\sum_{y \in A} Z_{N-1}(x)\right]^{2}=\left[\zeta_{N-1}(0)\right]^{2}
$$

Para $u=0$, o Lema 4.5.1 pode ser usado com os seguintes parâmetros:

$$
\begin{gathered}
x_{i}=x+2^{N-1}, x_{j}=2^{N-1}-y \\
a=2^{N}, \quad b=v^{-1 / 2}=2^{N}\left(\beta b_{N}\right)^{-1 / 2} \\
f\left(x_{i}\right)=\exp \left(2 v x^{2}\right) Z_{N-1}(x)
\end{gathered}
$$

para provar a validade da seguinte desigualdade

$$
\begin{aligned}
\zeta_{N}(0) & \geq e^{-1}\left[1+\left(\beta b_{N}\right)^{-1 / 2}\right]^{-1} \cdot\left[\sum_{s} \exp \left(2 v s^{2}\right) Z_{N-1}(s)\right]^{2} \\
& =e^{-1}\left[1+\left(\beta b_{N}\right)^{1 / 2}\right]^{-1} \cdot\left[\zeta_{N-1}(2 v)\right]^{2}
\end{aligned}
$$


Para calcular $L_{N}$ tome os logaritmos de (4.12) e (4.11) e os subtraia, chegando a:

$$
L_{N}(0)-L_{N}(-v) \geq 2\left[L_{N-1}(2 v)-L_{N-1}(0)\right]-1-\log \left[1+\left(\beta b_{N}\right)^{1 / 2}\right]
$$

A convexidade de $L_{N}(u)$ em $u$ implica em:

$$
\begin{gathered}
L_{N}(0)-L_{N}(-v) \leq v L_{N}^{\prime}(0) \\
L_{N-1}(2 v)-L_{N-1}(0) \geq 2 v L_{N-1}^{\prime}(0)
\end{gathered}
$$

Mas (4.16), (4.15) e (4.14) juntos nos dão uma desigualdade em $L_{N}^{\prime}$ e $L_{N-1}^{\prime}$ :

$$
\begin{aligned}
v L_{N}^{\prime}(0) & \geq L_{N}(0)-L_{N}(-v) \geq 2\left[L_{N-1}(2 v)-L_{N-1}(0)\right]-1-\log \left[1+\left(\beta b_{N}\right)^{1 / 2}\right] \\
& \geq 4 v L_{N-1}^{\prime}(0)-1-\log \left[1+\left(\beta b_{N}\right)^{1 / 2}\right]
\end{aligned}
$$

Lembrando que $f_{N}(N)=2^{-2 N} L_{N}^{\prime}(0)$, segue da desigualdade acima que

$$
f_{N}(N) \geq f_{N-1}(N-1)-\frac{1}{b_{N} \beta}\left\{1+\log \left(1+\left(\beta b_{N}\right)^{1 / 2}\right)\right\}
$$

Iterando a desigualdade acima e lembrando que $f_{0}(0)=1$ obtemos a seguinte estimativa

$$
f_{N}(N) \geq 1-\sum_{p=1}^{N}\left(b_{p} \beta\right)^{-1}\left\{1+\log \left(1+\left(\beta b_{p}\right)^{1 / 2}\right)\right\}
$$

Para tornar nossa análise mais simples, vamos decompor o somatório acima em duas partes, a depender no valor de $b_{p} \beta$. Antes de apresentar a decomposição observamos que a função

$$
\left(b_{p} \beta\right)^{-1}\left\{1+\log \left[1+\left(\beta b_{p}\right)^{1 / 2}\right]\right\}
$$

é decrescente em $b_{p} \beta$, para qualquer valor de $b_{p} \beta$ natural. Usando esse fato, vamos limitar os termos que satisfazem $b_{p} \beta \geq 9 p^{2}$ por

$$
\sum_{p=1}^{\infty}\left(9 p^{2}\right)^{-1}\{1+\log (1+3 p)\}<\frac{9}{16} .
$$

Como a função $\log (1+3 p)$ é crescente em $p$, os demais termos, $\operatorname{com} b_{N} \beta<9 p^{2}$, 
são limitados por:

$$
\sum_{p=1}^{\infty}\left(b_{p} \beta\right)^{-1}\{1+\log (1+3 p)\}
$$

Observando que para $p \geq 1$ temos

$$
1+\log [1+3 p]<\frac{7}{2} \log (1+p),
$$

podemos concluir que a desigualdade abaixo é verdadeira

$$
\sum_{p=1}^{\infty}\left(b_{p} \beta\right)^{-1}\{1+\log (1+3 p)\} \quad<\quad \beta^{-1} \sum_{p=1}^{\infty}\left(b_{p}\right)^{-1} \cdot \frac{7}{2} \log (1+p)=\frac{7}{2} L \beta^{-1} .
$$

Juntando as duas estimativas obtidas acima, para (4.17) ficamos com

$$
f_{N}(N) \geq 1-\left[\frac{9}{16}+\frac{7}{2} L \beta^{-1}\right]=\frac{7}{16}\left[1-8 L \beta^{-1}\right] .
$$

Então, $f_{N}(N)>0$ se $\beta>8 L$. Como a última desigualdade vale para todo $N$ e $m_{H}^{(2)}=\liminf _{N \rightarrow \infty} f_{N}(N)$, está provado o Teorema 4.5.2.

Agora voltamos a lembrar que um sistema Hierárquico com constantes $b_{p}=2^{(2-\alpha) p}$ tem acoplamento mais forte que um sistema linear com $J(|i-j|)=1 /|i-j|^{\alpha}$. Assim, calculando a constante $L$ para esse sistema Hierárquico, temos:

$$
L=\sum_{p=1}^{\infty}\left(b_{p}\right)^{-1} \log (1+p)=\sum_{p=1}^{\infty} 2^{(-2+\alpha) p} \log (1+p) .
$$

A quantidade acima evidentemente converge para $\alpha<2$, logo a magnetização quadrática é positiva nesses sistemas Hierárquicos. Por fim, essa estimativa para $L$ e os resultados da Seção 4.4 nos permitem concluir a demonstração do teorema abaixo.

Teorema 4.5.3. No modelo de Ising com constantes de acoplamento ferromagnéticas $J_{i j}=1 /|i-j|^{\alpha}$, com $1<\alpha<2$, existe magnetização espontânea positiva se

$$
\beta>8 \sum_{p=1}^{\infty} 2^{(-2+\alpha) p} \log (1+p) .
$$

O resultado da Seção 3.6 a respeito da dominância da magnetização 
de Currie-Weiss sobre sistemas somáveis nos permite concluir que esses modelos com $1<\alpha<2$ também têm uma temperatura crítica finita, acima da qual a magnetização é nula. 


\section{Referências Bibliográficas}

[1] G. Braga and M.R. Hilário, O teorema de perron-frobenius e ausência de transição de fase em modelos unidimensionais da mecânica estatística, Anais da Primeira Jornada de Iniciação Científica do IMPA (2005).

[2] L. Cioletti and A.O. Lopes, Interactions, specifications, dlr probabilities and the ruelle operator in the one-dimensional lattice, Preprint (2014), 1-41.

[3] L.M. Cioletti, C.C.Y. Dorea, and S. Vasconcelos da Silva, Diffusiveballistic transition in random polymers with drift and repulsive longrange interactions, Journal of Statistical Physics 156 (2014), no. 4, 760-765 (English).

[4] Simone Vasconcelos da Silva, Transição de fase difusiva-balística em polímeros aleatórios, Ph.D. thesis, Universidade de Brasília, 2014.

[5] Freeman J. Dyson, Existence of a phase-transition in a onedimensional Ising ferromagnet, Communications in Mathematical Physics 12 (1969), no. 2, 91-107.

[6] Richard S. Ellis, Entropy, large deviations, and statistical mechanics, Grundlehren der Mathematischen Wissenschaften [Fundamental Principles of Mathematical Sciences], vol. 271, Springer-Verlag, New York, 1985.

[7] Richard S. Ellis and James L. Monroe, A simple proof of the ghs and further inequalities, Comm. Math. Phys. 41 (1975), 33-38.

[8] Richard S. Ellis and Charles M. Newman, Necessary and sufficient conditions for the GHS inequality with applications to analysis and probability, Trans. Amer. Math. Soc. 237 (1978), 83-99. 
[9] C. M. Fortuin, P. W. Kasteleyn, and J Ginibre, Correlation inequalities on some partially ordered sets, Communications in Mathematical Physics 2 (1971), no. 22, 89-103.

[10] Jürg Fröhlich and Thomas Spencer, The phase transition in the onedimensional Ising model with $1 / r^{2}$ interaction energy, Communications in Mathematical Physics 84 (1982), no. 1, 87-101.

[11] Andrew Gelman, Jonathan N. Katz, and Francis Tuerlinckx, The mathematics and statistics of voting power, Statistical Science 17 (2002), no. 4, 420-435.

[12] Hans-Otto Georgii, Gibbs measures and phase transitions, second ed., de Gruyter Studies in Mathematics, vol. 9, Walter de Gruyter \& Co., Berlin, 2011.

[13] R.B. Griffiths, Correlations in ising ferromagnets. I, Journal Mathematical Physics 3 (1967), no. 8, 478-483.

[14] Robert B. Griffiths, C. A. Hurst, and S. Sherman, Concavity of magnetization of an Ising ferromagnet in a positive external field, J. Mathematical Phys. 11 (1970), 790-795.

[15] Ernst Ising, Beitrag zur theorie des ferromagnetismus, Ph.D. thesis, 1929.

[16] D. G. Kelly and S. Sherman, General griffhts inequalities on correlations in ising ferromagnets, Journal of Mathematical Physics 9 (1969), 466-484.

[17] Joel L. Lebowitz, GHS and other inequalities, Comm. Math. Phys. 35 (1974), 87-92.

[18] Paul A. Pearce, Mean-field bounds on the magnetization for ferromagnetic spin models, J. Statist. Phys. 25 (1981), no. 2, 309-320.

[19] R. Peierls, On ising's model of ferromagnetism, Mathematical Proceedings of the Cambridge Philosophical Society 32 (1936), no. 3, 477-481.

[20] R. Tyrrell Rockafellar, Convex analysis, Princeton Mathematical Series, No. 28, Princeton University Press, Princeton, N.J., 1970. 
Referências Bibliográficas

[21] D. Ruelle, Statistical mechanics of a one-dimensional lattice gas, Communications in Mathematical Physics 9 (1968), 267-278. 